Aus der Klinik für Mund-, Kiefer- und Gesichtschirurgie

(Prof. Dr. med. Dr. med. dent. H. Schliephake)

im Zentrum Zahn-, Mund- und Kieferheilkunde

der Medizinischen Fakultät der Georg-August-Universität Göttingen

\title{
Magnetresonanztomographische und manometrische Untersuchungen zur Verlagerung oropharyngealer Strukturen während des Saugschluckvorgangs
}

\section{INAUGURAL - DISSERTATION}

\author{
zur Erlangung des Doktorgrades \\ für Zahnheilkunde \\ der Medizinischen Fakultät der \\ Georg-August-Universität zu Göttingen
}

vorgelegt von

Johannes Glombek

aus

Viersen

Göttingen 2014 
Dekan:

I. Berichterstatter:

II. Berichterstatter/in:

III. Berichterstatter/in:
Prof. Dr. rer. nat. H. K. Kroemer

Prof. Dr. Dr. Wilfried Engelke

Prof. Dr. Martin Canis

7. Oktober 2014 


\section{Inhaltsverzeichnis}

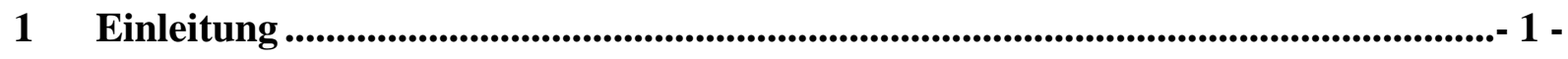

1.1 Anatomische Aspekte des orofazialen Systems …............................................ 1 -

1.1.1 Biofunktionelle Einheiten .................................................................. 3 -

1.1.2 Biofunktionelle Kompartimente ................................................................. 4 -

1.1.3 Biofunktionelle Verschlüsse .................................................................... 5 -

1.2 Funktionelle Aspekte des orofazialen Systems ….............................................. 6 -

1.2.1 Ruhelage und Zungenpositionierung …................................................... 7 -

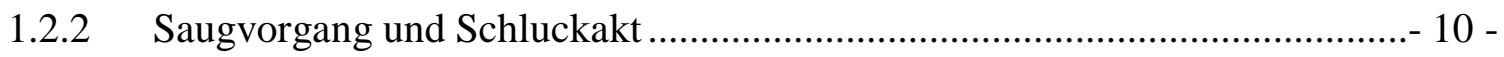

1.2.2.1 Die orale Präparationsphase ........................................................... 10 -

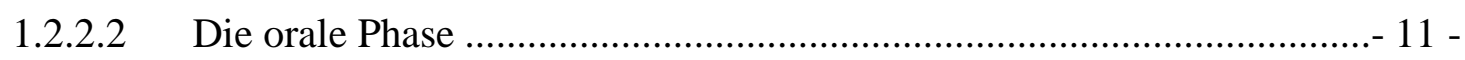

1.2.2.3 Die pharyngeale Transportphase ........................................................ 11 -

1.2.2.4 Die ösophageale Transportphase .................................................... 12 -

1.3 Methoden zur Untersuchung der Saug- und Schluckfunktion..............................- 13 -

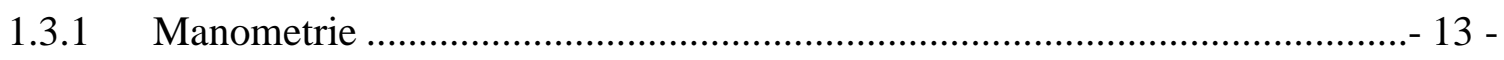

1.3.2 Videofluoroskopie und Videokinematographie ......................................... 16 -

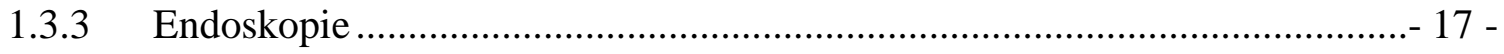

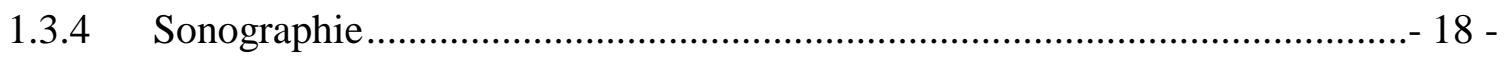

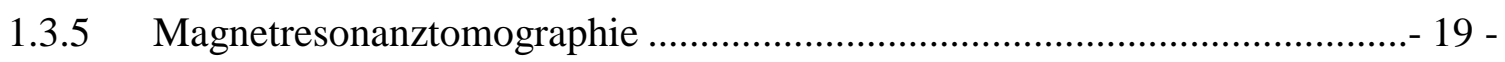

1.3.6 Manofluorographie und Manovideoendoskopie ......................................... 22 -

1.4 Fragestellungen und Zielsetzung der Arbeit................................................... 23 -

2 Material und Methode .................................................................................................................- 24 -

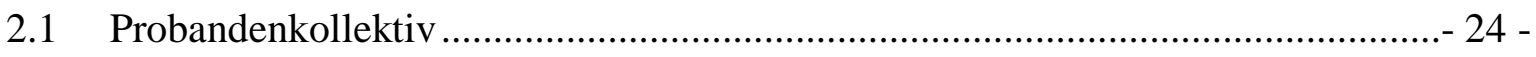

2.1.1 Auswahl der Probanden ............................................................................. 24 -

2.1.2 Aufklärung der Probanden ................................................................... 25 -

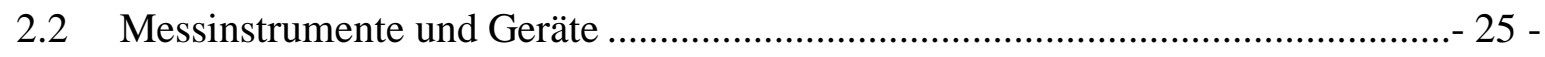

2.2.1 Intraoraler Anteil des Applikations- und Messsystems................................. 25 -

2.2.2 Extraoraler Anteil des Applikations- und Messsystems ................................ 27 -

2.2.3 Räumlicher Aufbau des Messsystems .........................................................- 28 -

2.2.4 Manometer .................................................................................... 29 - 


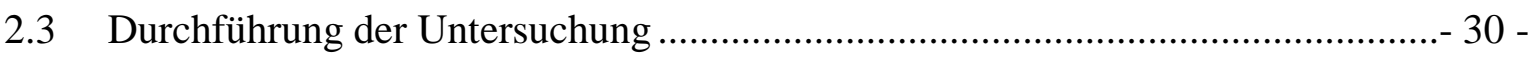

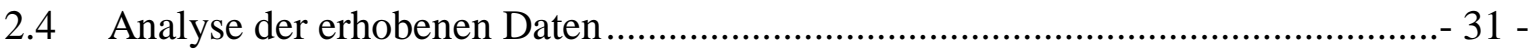

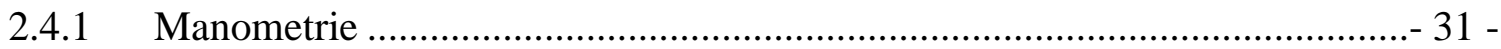

2.4.2 Magnetresonanztomographie ................................................................. 33 -

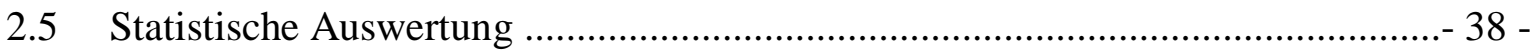

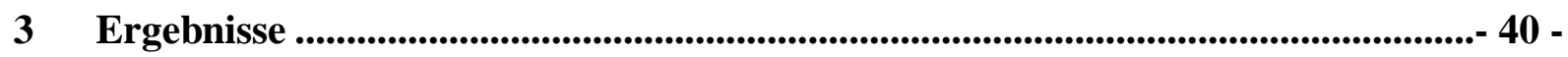

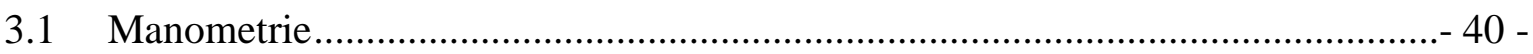

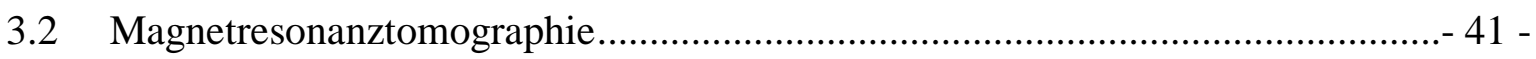

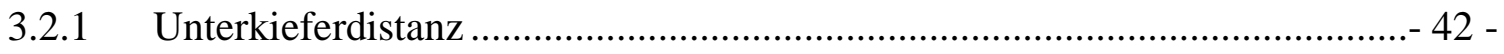

3.2.2 Saugraum (Subpalatinales Kompartiment / BFK 2) .................................... 43 -

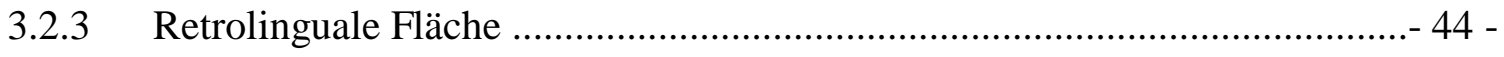

3.2.4 Epipharyngeale Fläche …............................................................................. 45 -

3.2.5 Externe Mundbodenfläche ….......................................................................- 46 -

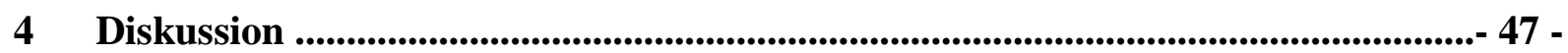

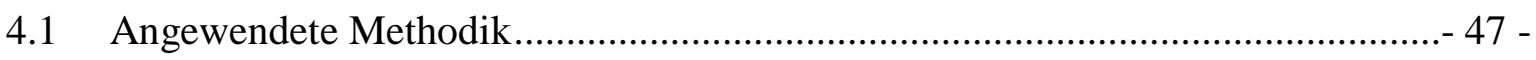

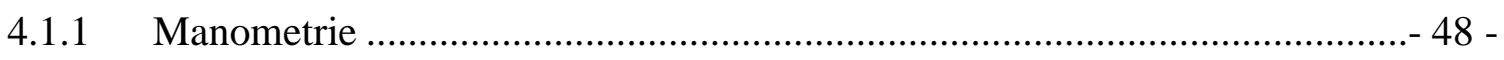

4.1.2 Bildgebende Verfahren............................................................................. 49 -

4.1.3 Bewertung des bimodalen Verfahrens ......................................................... 54 -

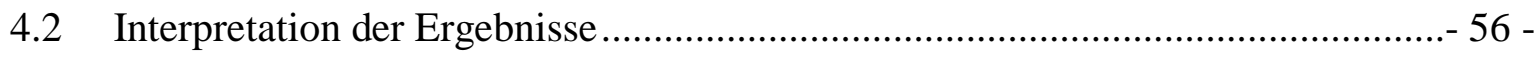

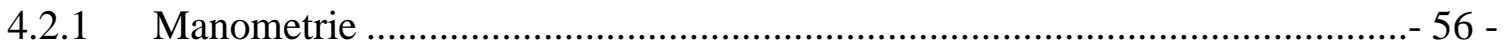

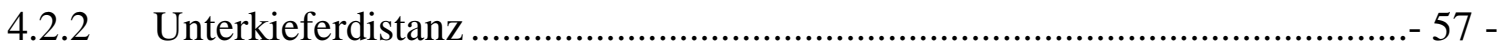

4.2.3 Saugraum (Subpalatinales Kompartiment / BFK 2) .................................... 57 -

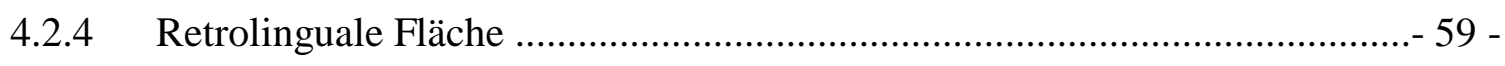

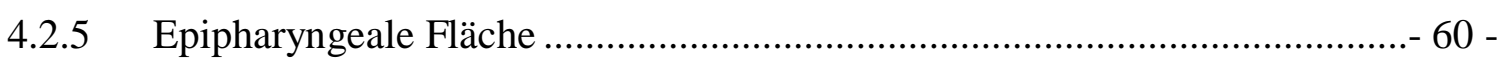

4.2.6 Externe Mundbodenfläche ……........................................................... 61 -

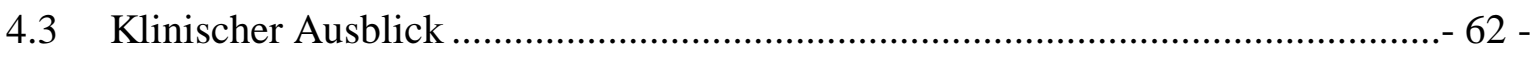

5 Zusammenfassung ............................................................................................ 63 -

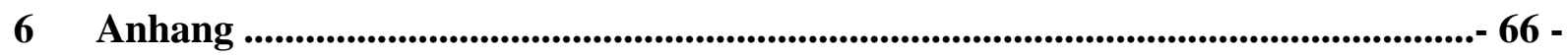

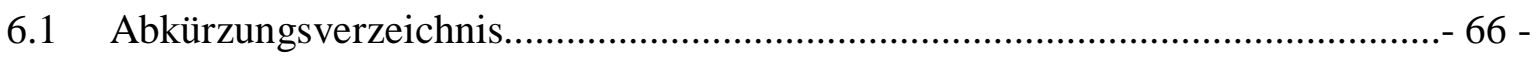




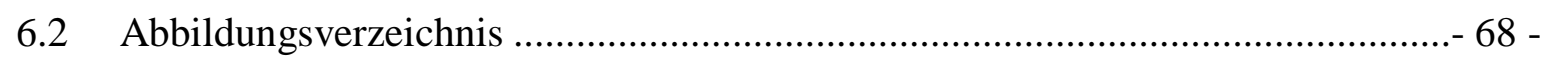

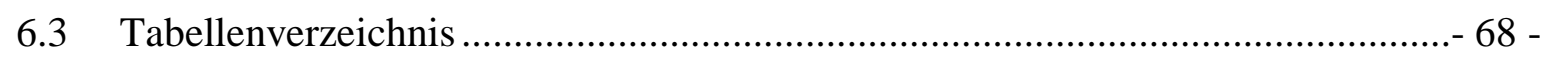

6.4 Unterlagen zur ethischen Begutachtung ........................................................ 69 -

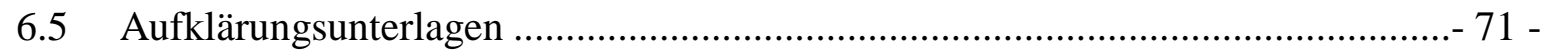

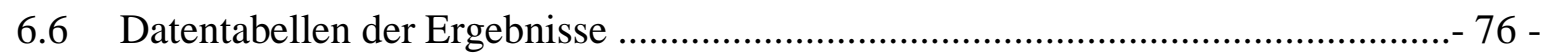

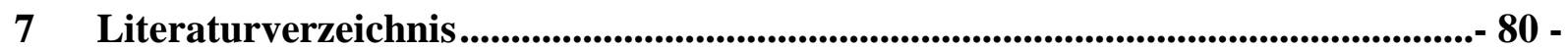




\section{Einleitung}

Der Mechanismus der Nutrition erfolgt in einem komplexen Zusammenspiel einzelner anatomischer Strukturen innerhalb des orofazialen Systems. Saug- und Schluckvorgänge zu erfassen und einzuordnen erfordert Grundkenntnisse über den orofazialen anatomischen Aufbau und die auf ihm beruhenden Funktionen.

\subsection{Anatomische Aspekte des orofazialen Systems}

Das orofaziale System besteht im Allgemeinen aus der Mundhöhle, den Nasenhöhlen und dem Rachen sowie den Strukturen, die diese Räume umgeben. Dazu zählen Ober- und Unterkiefer mit Gaumen und Zähnen sowie die Kiefergelenke. Lippen, Wange, Zunge und Mundboden stellen wichtige Teile der orofazialen Muskulatur dar. Auch die mimische Muskulatur ist dem orofazialen System zugeordnet. Der Kehlkopf bildet den Abschluss des orofazialen Systems in aboraler Richtung.

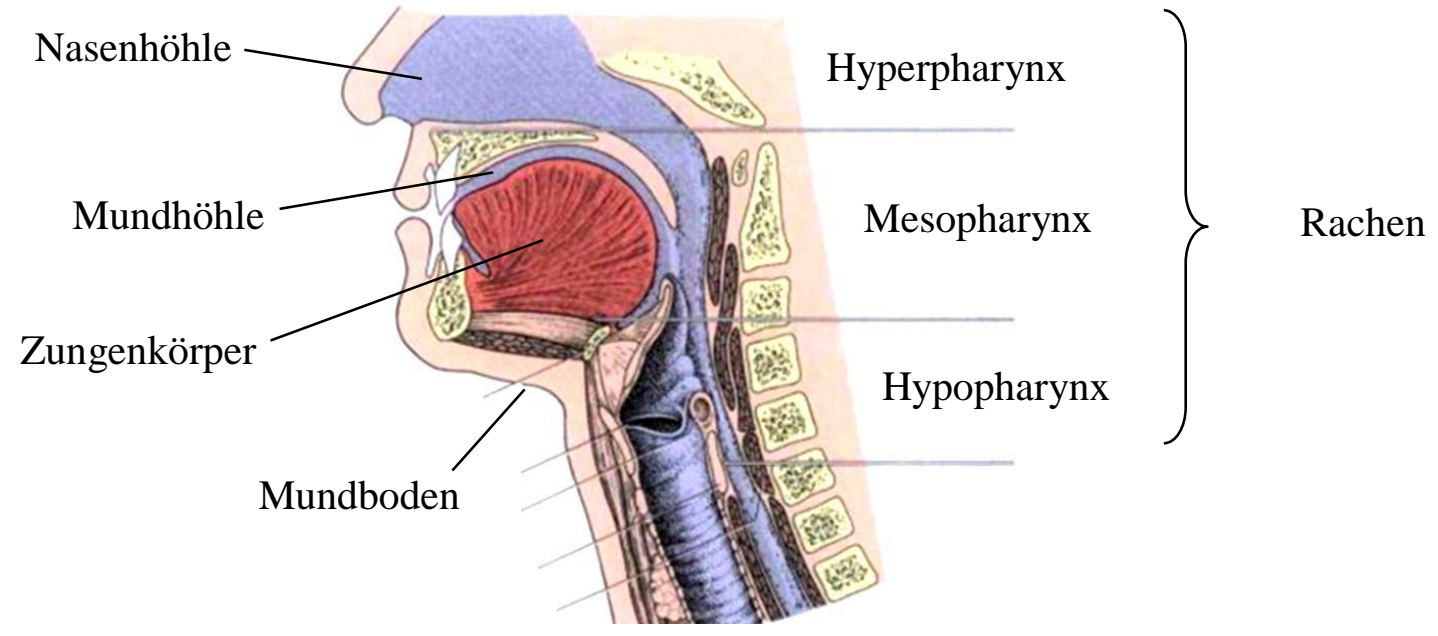

Abbildung 1 Schema der orofazialen Anatomie (nach: Bartolome und Schröter-Morasch 2010, S. 2) 
Die eigentliche Mundhöhle (cavum oris proprium) wird vom Zahnbogen nach außen hin begrenzt. Außerhalb dessen liegt der Mundvorhof (vestibulum oris), der bis zu den Lippen nach ventral und zur Wangenschleimhaut nach lateral reicht (Lippert 2006). Der Mundboden (diaphragma oris) bildet den kaudalen Abschluss der Mundhöhle. Er ist überwiegend muskulär aufgebaut und besteht aus den musculi geniohyoideus, digastricus et mylohyoideus. Insbesondere letzterer ist dafür verantwortlich, dass der Mundboden einerseits zwar auf einer stabilen Basis fußt, andererseits aber auch eine Verformbarkeit erfährt, die mit den orofazialen Funktionen eng verknüpft ist. Der Gaumen begrenzt die Mundhöhle nach kranial und grenzt sie dadurch von der Kieferhöhle ab. Er ist im vorderen, größeren Anteil knöchern, im hinteren Anteil weichgeweblich konstituiert. Dort befindet sich das velum palatinum, das ebenfalls aufgrund diverser Formationsmöglichkeiten ein Konglomerat aus Muskeln darstellt. Der $m$. tensor veli palatini spannt das Gaumensegel, sorgt aber auch für die Mittelohrbelüftung über die tuba auditiva. Der $m$. levator veli palatini ist vor allem für die Hebung des Gaumensegels verantwortlich. Während dieser Funktion spannt sich das Gaumensegel zwischen hartem Gaumen und Pharynxwand auf, wodurch der Naso- vom Oropharynx verschlossen wird. Gleichzeitig ist dabei ein weiterer Muskel, der $m$. constrictor pharyngis superior, aktiv. Die musculi palatoglossus et palatopharyngeus bilden die zwei Gaumenbögen, die sich lateral vom Gaumensegel erstrecken. Sie laufen kranial an der uvula zusammen, die in Ruhelage Kontakt mit der Zunge hat. Für die Beweglichkeit der uvula ist der $m$. uvulae ausschlaggebend.

Die Zunge als zentraler, muskulärer Körper innerhalb der Mundhöhle ist wesentlich an den Vorgängen Saugen, Schlucken und Kauen beteiligt. Ist der Unterkiefer adduziert, füllt der mit Mukosa überzogene Zungenmuskel die Mundhöhle fast im Gesamten aus.

Die Zungenwurzel (Zungengrund) ist am Zungenbein (os hyoideum) befestigt und befindet sich im dorsalen Teil der Zunge, in dem sie besonders voluminös ist. In Ruhelage kann ein Kontakt der posterioren Zunge zur Rachenhinterwand bestehen. Der Zungenrücken zeigt nach kranial und bildet die Oberseite der Zunge, die durch ihre Wölbung und - je nach Aktivitätszustand - durch ihre Prominenz imponieren kann. Hier ist in Ruheposition der Kontakt zur uvula möglich. Der sulcus medianus verläuft in der Mitte des Zungenrückens in Längsrichtung als Furche. Durch Bänder und Muskeln steht die Zunge in Kontakt zu umgebenden Strukturen. Es werden die äußeren Zungenmuskeln von der Zungenbinnenmuskulatur unterschieden. Zur äußeren Zungenmuskulatur zählen die musculi hyo-, stylo- et genioglossi, die die Zunge über ihre Muskelansätze an umliegenden Strukturen 
wie dem Zungenbein, dem processus styloideus oder auch der Epiglottis fixieren (Lippert 2006). Dadurch kann die Zunge auf höchst variable Weise in allen Raumebenen bewegt werden.

Die Binnenmuskulatur hingegen verleiht dem Zungenkörper eine große interne Verformbarkeit. $\mathrm{Zu}$ nennen sind hier die musculi longitudinales inferior et superior sowie die musculi transversus et verticalis. In ihrem Zusammenspiel sorgen sie vor allem für die funktionsabhängige Verlängerung und Verkürzung der Zunge (Dauber und Feneis 2005). Sie befähigen diese dadurch, innerhalb der Mundhöhle funktionelle Verschlüsse und Räume zu bilden. Insbesondere diese Eigenschaften sind im Rahmen des Saugvorgangs von Bedeutung.

Die Bestandteile des orofazialen Systems eines Menschen wechselwirken auf physiologische Weise miteinander. Das orofaziale System wird nach Engelke (2011) in funktionelle Einheiten, Kompartimente und Verschlüsse gegliedert. Auf dieser Grundlage können Anatomie und Funktion systematisch beschrieben werden.

Das orofaziale Organ kann demnach als ein Hohlraumsystem betrachtet werden, welches seine strukturelle Einteilung durch Mund, Nase und Pharynx sowie angrenzende viszerokranielle Strukturen je nach biofunktionellem Zustand erhält. Der verwendete Begriff der „Funktionseinheiten“ (Engelke 2009, S.3) innerhalb des Biofunktionellen Modells bringt zum Ausdruck, dass die Funktion als Grundlage für die Einteilung herangezogen wurde, worin ein wesentlicher Unterschied zu herkömmlichen, eher deskriptiven Definitionen besteht. Es werden 12 Biofunktionelle Einheiten (BFE) des orofazialen Systems unterschieden und 4 Biofunktionelle Kompartimente (BFK) voneinander differenziert. Außerdem lassen sich 7 Verschlusssysteme definieren (BFV), die die Kompartimente voneinander abgrenzen.

\subsubsection{Biofunktionelle Einheiten}

Bei den Biofunktionellen Einheiten handelt es sich um die anatomischen Komponenten des orofazialen Systems, die größtenteils Wandstrukturen des Hohlraumsystems sind. Die Einheiten 1 und 2 stellen die Ober- und die Unterlippe mit der angrenzenden Muskulatur dar, die insbesondere vom $m$. orbicularis oris gebildet wird. Der Oberkiefer und der harte Gaumen bilden die Einheit 3, die die kaudale Nasenhöhlenbegrenzung sowie die kraniale Mundhöhlenbegrenzung ist. Einheiten 4 und 5 beschreiben die Zahnbögen des Ober- und des Unterkiefers. 
Der Unterkiefer selbst mit den ansetzenden Kaumuskeln bildet Einheit 6. Zentrale Struktur innerhalb der Mundhöhle ist die Zunge (Einheit 7), die in eine anteriore (7a) und eine posteriore (7b) Portion unterteilt wird. Einheit 8 definiert das Gaumensegel sowie die Gaumenbögen. Die Nasenhöhle und der obere Pharynxbereich sind als Einheit 9 zusammengefasst, da sie funktionell eine Einheit bilden. Angrenzend befindet sich die Einheit 10, der Mesopharynx, in dem respiratorische und nutritive Funktionen stattfinden, was in Einheit 9 nur pathologisch auftreten kann. Der Kehlkopf mit Eingang in den Larynx bildet die Einheit 11. Die letzte Einheit bilden die Kiefergelenke (Einheit 12). Einen Überblick über die Definitionen und Lokalisationen verschafft Abbildung 2.

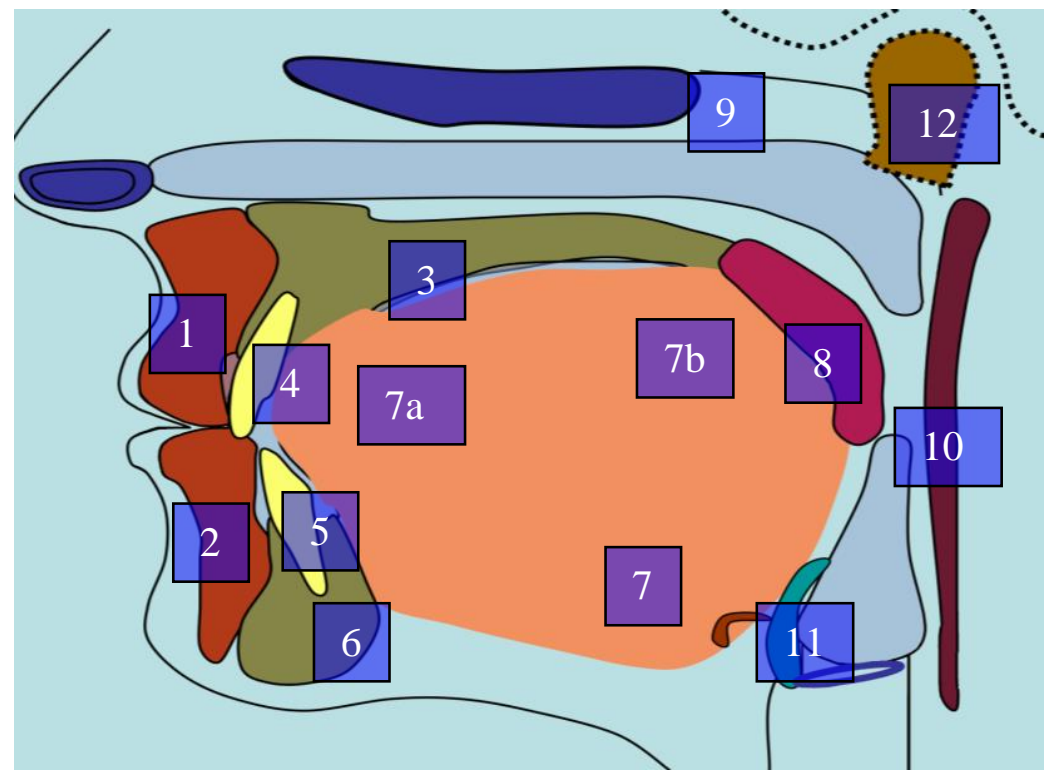

Abbildung 2 Biofunktionelle Einheiten im schematisierten Mediosagittalschnitt (nach Engelke 2009, S.2): 1 Oberlippe und angrenzende mimische Muskulatur; 2 Unterlippe und angrenzende Wangenmuskulatur; 3 Oberkiefer und harter Gaumen; 4 Oberkieferzahnbogen; 5 Unterkieferzahnbogen; 6 Unterkiefer einschließlich Kaumuskulatur; 7 Zunge einschließlich Zungenbein und angrenzende Muskeln; 7a anteriore, 7b posteriore Zunge; 8 Gaumensegel einschließlich Gaumenbögen; 9 Nase und Epipharynx; 10 Mesopharynx; 11 Larynxeingang; 12 Kiefergelenke

\subsubsection{Biofunktionelle Kompartimente}

Das Biofunktionelle Kompartiment 1 (BFK 1) wird als interokklusaler Raum beschrieben. Es bezieht sich auf den Raum, der nach außen durch Lippen und Wangen, nach innen / oral 
durch die Zunge bzw. ihre Unterseite begrenzt wird. In ihm liegen physiologisch die Zahnreihen, er wird auch als Kauschlauch bezeichnet (Engelke 2007).

Das Biofunktionelle Kompartiment 2 (BFK 2) bezeichnet den subpalatinalen / palatolingualen Raum, der von Zungenrücken und Gaumen nach jeweils kaudal bzw. kranial begrenzt ist. Über das Velum als dorsaler Begrenzung geht BFK 2 in BFK 3 über.

Das Biofunktionelle Kompartiment 3 (BFK 3, mesopharyngeales Kompartiment) definiert den Raum, der im velopharyngealen Sphinkter nach kranial und in der Glottis bzw. dem Larynxeingang nach kaudal seine Begrenzungen findet.

Das Biofunktionelle Kompartiment 4 (BFK 4, nasoepipharyngeales Kompartiment) besteht aus den nasalen Luftwegen und dem Epipharynx. Es kann nur zum Mesopharynx hin dicht geschlossen werden.

\subsubsection{Biofunktionelle Verschlüsse}

Bei den Biofunktionellen Verschlüssen (BFV) handelt es sich um Kontaktbereiche zwischen Biofunktionellen Einheiten. Engelke (2009) definiert den ersten Biofunktionellen Verschluss als denjenigen, der zwischen Ober- und Unterlippe stattfindet. BFV 2 entspricht dem Verschluss zwischen Oberkiefer und vorderem Zungenanteil. Verschluss 3 ist der velolinguale Verschluss, an dem die posteriore Zunge sowie das Velum Anteil haben. Die kraniale Begrenzung von BFK 3 bildet der vierte Biofunktionelle Verschluss, es handelt sich um den velopharyngealen Sphinkter, der aus dem Velum und Teilen der Pharynxwände gebildet wird. Der fünfte Verschluss entspricht der Glottis, die mit dem BFV 6 (Eingang des Ösophagus) das orofaziale System nach kaudal abschließt. BFV 7 ist der Naseneingang und damit ein Verschluss, der zwar durch eine gewisse Öffnungsvarianz den Durchstrom von Atemluft regulieren, jedoch niemals einen dichten Verschluss darstellen kann. 


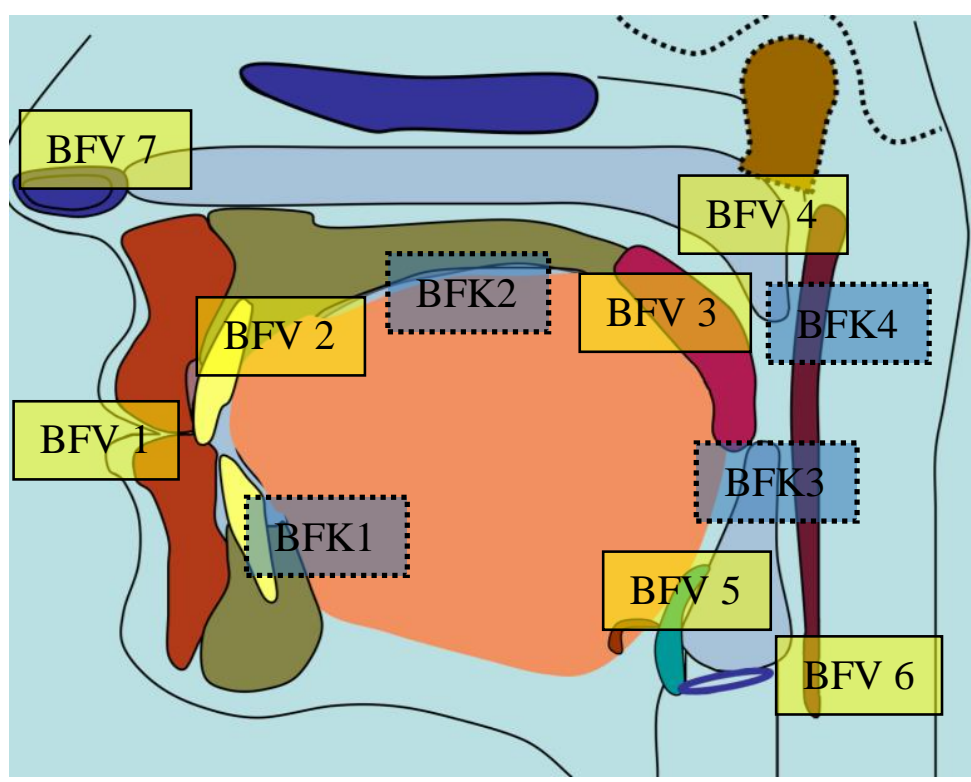

Abbildung 3 Biofunktionelle Verschlüsse (BFV) und Kompartimente (BFK) im schematisierten Mediosagittalschnitt (nach Engelke 2009, S.3); Definitionen s. Text

\subsection{Funktionelle Aspekte des orofazialen Systems}

Die Funktionen des orofazialen Systems stellen die Grundlage aller lebensnotwendigen Aktivitäten dar: Atmung und Nahrungsverarbeitung stehen dabei im Vordergrund der Vitalfunktionen. Zu der Nahrungsverarbeitung zählen die Funktionen Saugen, Kauen und Schlucken. Die Lautbildung sowie physiognomische Funktionen gehören zu den kommunikativen bzw. ästhetischen Aufgaben des orofazialen Systems (Reitemeier et al. 2006). Weiterhin werden dem orofazialen System Merkmale zuteil, die im Zusammenhang mit Sensorik und Sensibilität stehen und damit die Grundlage für die Empfindung von äußeren Einflüssen darstellen (Friedrich und Bigenzahn 1995).

Eine nähere Betrachtung der orofazialen Funktionen ist insbesondere deshalb von Bedeutung, da Störungen in den fein-koordinierten Abläufen zur massiven Beeinträchtigung der Vitalaktivitäten führen können. Dies ist vor allem auf das Saugen, Schlucken und Atmen zu beziehen. In entwicklungsgeschichtlicher Hinsicht kann deren biologischer Stellenwert schon daran festgemacht werden, dass sie $\mathrm{zu}$ den am tiefsten verankerten und am weitesten zurückreichenden Funktionen des Individuums zählen. Ohne die Atmung zur Sauerstoff- bzw. die Ernährung zur Energiezufuhr können keine Vitalfunktionen stattfinden. Koordinations- 
störungen im Bereich der anatomischen Strukturen, die für die Nutrition verantwortlich sind, führen demnach häufig zu einer stark reduzierten Lebensqualität.

\subsubsection{Ruhelage und Zungenpositionierung}

In Ruhe sollen sich die Strukturen des orofazialen Systems im Gleichgewicht befinden. Dieses Gleichgewicht kann als Impulsgleichgewicht verstanden werden, bei dem die Biofunktionellen Einheiten des orofazialen Systems Kräfte gegeneinander ausüben, die sich ausgleichen. Dabei wird der Zunge als zentralem Organ innerhalb der Mundhöhle eine Schlüsselrolle zuteil (Lear und Moorrees 1969), die wesentlich auf ihren vielfältigen Bewegungsmöglichkeiten beruht. Schon zu Beginn des 20. Jahrhunderts wurde eine Balance zwischen einer den Wangen und den Lippen entgegengesetzt wirkenden Zungenkraft beschrieben (Angle 1907), die grundlegend für kieferorthopädisches Verständnis ist. Auch Abrams (1963) und Mew (2004) beschrieben kompensierende Kräfte zwischen Zunge, Wangen und Lippen. Die Bedeutsamkeit von Kraftgröße und Einwirkdauer der am System beteiligten Gleichgewichtsfaktoren betont Proffit (1978) in seiner theory of equilibrium. Einige Jahre später nimmt Tränkmann (1982) die Unterteilung in einen äußeren Funktionskreis (u.a. mimische Muskeln und Teile der Kiefermuskulatur) und einen inneren Funktionskreis (Zungen- und Unterzungenmuskulatur) vor. $\mathrm{Er}$ betrachtet orofaziale Dyskinesien als Fehlfunktionen zwischen beiden. Impulse der beiden Funktionskreise sollten sowohl in Funktion als auch im Ruhezustand im Gleichgewicht sein. Hierzu müssten Belastungen durch die jeweils andere Muskelgruppe ausgeglichen werden. Muskuläre Ungleichgewichte hingegen (z.B. Habits) beeinträchtigten das balancierte Zusammenspiel des orofazialen Systems. Melsen et al. (1987) betonen diese Aspekte erneut in kieferorthopädischer Hinsicht.

Zur Ruhelage des orofazialen Systems tragen im Wesentlichen verschiedene Zungenpositionen bei. Häufig wurde in Anbetracht des anatomisch-muskulären Zungenaufbaus die Einnahme dieser Positionen als ausschließlich neuromuskulär angenommen (Ballard 1966). Hierbei liegen rein neuronale Mechanismen als Impuls zur Aufrechterhaltung eines Muskeltonus der Zunge in Ruhe zugrunde. Körbitz (1914) wählte zuvor einen anderen Ansatz zur Beschreibung der Zungenpositionen, der in späteren Arbeiten wieder aufgegriffen wurde (Noltemeier 1949, Eckert-Möbius 1953, Breustedt 1970). Körbitz beschreibt eine Ruhelage der am Gaumen anliegenden Zunge mit geschlossenem Mund, die 
vornehmlich durch vorheriges Schlucken mit konsekutivem Aufbau von Unterdruck in der Mundhöhle eingenommen und unterhalten wird. Dabei bestehen Saugräume zwischen Zunge und hartem Gaumen, die durch eine Entleerung des Mundinnenraumes nach dem Schlucken aufgebaut werden. Engelke unterscheidet - ähnlich Fränkel (1967) - im Biofunktionellen Modell eine offene und eine geschlossene Ruhelage voneinander: Die offene Ruhelage ist gekennzeichnet durch frei bewegliche anatomische Einheiten bei offenen Verschlusssystemen ohne vorherigen Schluckakt. Die intraoralen Drücke entsprechen dabei dem atmosphärischen, extra-orofazial herrschenden Druck. Bei der geschlossenen Ruhelage hingegen, die durch einen betonten Schluckakt eingenommen wird, sind überwiegend suffiziente Verschlüsse ausgebildet, der Zungenrücken ist dem Gaumen angelagert und formt das subpalatinale Kompartiment zu einem Saugraum, in dem - ähnlich Körbitz (1914) und Noltemeier (1949) Unterdrücke beschrieben werden. Eckert-Möbius (1953) beobachtete röntgenologisch nach einfachem Mundschluss ohne Schlucken luftgefüllte Hohlräume zwischen Zunge und Gaumen im Sinne einer offenen Ruhelage, während Wein et al. (1988) eine Schluckinduzierte, vollständige Zungenanlagerung an den Gaumen sonographisch nachwiesen. Engelke et al. (1995a) zeigten in elektromagnetischen, artikulographischen Untersuchungen zur Koordination von Velumbewegungen und Zungenrücken ebenfalls Kontaktpositionen von Zunge und Gaumen nach dem Schluckakt und beobachteten dabei eine anterokaudale Velumposition. Auf Polysensographie beruhende Erhebungen durch Engelke und Hoch (1999) ergeben weitere Hinweise auf Schluck-induzierte, Gaumen-orientierte Zungenbewegungen.

Die postdeglutorische Einnahme der biofunktionellen Ruhelage ist somit Resultat der orofazialen Funktionen Saugen und Schlucken, die primär durch die Zunge - als zentraler Struktur innerhalb eines Systems interagierender Einheiten - ausgeführt werden. Damit bekommen die Funktionen Saugen und Schlucken nicht nur im Zusammenhang der Nahrungsverarbeitung eine wichtige Bedeutung, sondern spielen auch für die Einnahme einer Ruhelage, in der sich die Strukturen zu etwa $85 \%$ befinden (Zirkler 2002), eine besondere Rolle.

Auf dieser Basis wurden Versuche entwickelt, diese gaumennahe Zungenruhelage als Resultat eines Saugschluckvorgangs kontrolliert einzunehmen. In Anlehnung an die beschriebene Arbeit von Körbitz (1914) zeigt Engelke (2003) mithilfe einer Membrantrichterplatte, dass die Einnahme der Zungenposition am harten Gaumen kontrolliert zu erzielen ist. Dabei handelt es sich um das so genannte druckkontrollierte Zungenrepositionsmanöver (ZRM). 


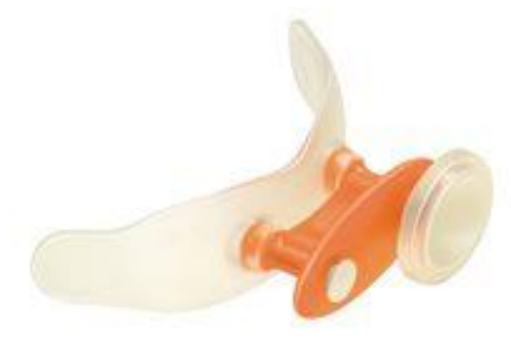

Abbildung 4 Membrantrichterplatte, bestehend aus einer Mundvorhofplatte und einer extraoralen Druckanzeige als Vakuumaktivator (Engelke 2009, S.4)

Die Bildung eines intraoralen Unterdrucks nach dem Schlucken kann durch eine extraoral einsehbare Membran kontrolliert werden, indem durch eine Röhre, die durch den Lippenverschluss verläuft, eine kommunikative Verbindung zwischen intra- und extraoralem Druckmilieu geschaffen wird. Diese Röhre hat die Form eines Trichters, über dessen Öffnung, die nach extraoral gerichtet ist, die Membran gespannt ist. Der Trichter wird an einer Mundvorhofplatte angebracht. Zunächst für Erwachsene entwickelt, dann auch als „SchnarchSchnuller“ für Kinder eingeführt, kann mit der Membrantrichterplatte erst unter direkter, ärztlicher Anleitung, später auch autodidaktisch zu Hause, die postdeglutorische Zungenreposition an den Gaumen mit Unterdruckbildung eingeübt werden. Radiologisch lässt sich die Annäherung der Zunge an den harten Gaumen, einhergehend mit einer linguovelaren Verschlussbildung, durch das Zungenrepositionsmanöver zeigen. Diese Position entspricht einer Ruhelage im funktionellen Gleichgewicht. Die Herstellung des orofazialen Äquilibriums stellt sich als Resultat des Saug- und Schluckvorganges dar und bietet gleichzeitig für die respiratorische Funktion den optimalen Zustand, da das Velum durch den intraoralen Unterdruck am Zungengrund stabilisiert wird. 


\subsubsection{Saugvorgang und Schluckakt}

Der Vorgang des Saugens ist in den allermeisten Fällen als unmittelbare Vorbereitung für einen Schluckakt anzusehen. Beide Vorgänge stellen essentielle Mechanismen dar, die auf neuromuskulärer Grundlage den Transport eines Nahrungsbolus zum Ösophagus durchführen. Logemann (1988) zufolge ist das Saugen in die erste Phase des Schluckvorgangs einzuordnen. Anhand von Untersuchungen an Säuglingen (Delaney und Arvedson 2008) sowie mithilfe von tierexperimentellen Versuchen (Thexton et al. 2004) wurde der Saugvorgang näher beschrieben, Studien auf Grundlage von Forschungen am erwachsenen Menschen, bei denen die Koordination der partizipativen Elemente während des physiologischen Saugens untersucht wurde, liegen allerdings in lediglich unzureichender Form vor. Während die Existenz negativer Drücke beim Saug- und Schluckvorgang unumstritten ist (Nilsson et al. 1996, Kieser et al. 2008, Kennedy et al. 2010), gilt die Beteiligung der orofazialen Kompartimente und Strukturen während des Saugens als weitgehend ungeklärt.

Der meist unbeachtet erfolgende Saugvorgang mit anschließendem Schluckakt bezeichnet eine komplexe Bewegungsabfolge vieler Muskeln im oropharyngealen Bereich, die dazu dient, Nahrung und Speichel aus der Mundhöhle in den Magen zu befördern. Dabei muss eine Koordination mit den Luftwegen stattfinden (Matsuo und Palmer 2009).

Hilfreich zur genaueren Betrachtung des Saug- und des Schluckvorgangs ist die Unterteilung in verschiedene, von Magendie ursprünglich definierte und durch Logemann später ergänzte, Phasen (Magendie 1813, Logemann 1988), die allerdings fließende Übergänge zeigen (Böhme 2003).

\subsubsection{Die orale Präparationsphase}

Die in der oralen Präparationsphase ablaufenden Vorgänge gehen dem eigentlichen Schluckakt voran, sind zudem aber zwingend für ihn erforderlich. Dazu zählen Lippenschluss, Kieferbewegung, Nahrungszerkleinerung, Durchmischung mit Speichel und Portionierung des Bolus (Logemann 1988), der sich im subpalatinalen Kompartiment befindet. Letzteres geschieht durch höchst präzise, neuromuskulär kontrollierte Bewegungen der Zunge, die sich löffelartig um den Bolus herum anlegt (Anagnostara et al. 2001) und ihn auf diese Weise formt. Gingrich et al. (2012) sprechen von einer kolbenartigen, propulsiven Fortbewegung des Bolus durch die Zunge, die je nach Bolusviskosität unterschiedlich hohe Drücke generiert. 
Nach Logemann wölbt sich derweil das Velum nach anterior (velolingualer Verschluss nach aboral), um ein vorzeitiges Hineingleiten des Bolus in den Pharynx zu vermeiden (Logemann 1988). Die zentrale Rolle des Kauens für das orale System wird im Zusammenhang mit der Nahrungszerkleinerung deutlich (Orchardson und Cadden 2009). Durch die Zugehörigkeit des Beißens und Kauens zur oralen Präparationsphase ist die Dauer sehr unterschiedlich (Böhme 2003) und nebenbei stark methodenabhängig (Engelke et al. 1995b). Der Vorgang des Saugens spielt beim flüssigen Bolus eine zentrale Rolle in der Zungen-koordinierten Präparation des Schluckakts.

\subsubsection{Die orale Phase}

Die Lippen sind geschlossen, es kann dabei weitergekaut werden. Unter gleichzeitiger Anspannung des Wangenmuskels bewegt sich die Zunge zum Start des Schluckvorgangs nach kranial und dorsal und drückt damit gegen den harten Gaumen (Logemann 1988; Engelke et al. 1995b), Funktionsraum 2 wird damit verkleinert. Der Bolus wird durch die Schlundenge nach pharyngeal verdrängt, Larynx und Hyoid heben sich (Engelke et al. 1995b). Ist dies noch weitestgehend willkürlicher Kontrolle unterlegen, verselbständigen sich im Folgenden die Abläufe durch den Kontakt zu sogenannten Triggerarealen (Böhme et al. 2006): Sofern der Speisebrei den Zungengrund oder die dorsale Pharynxwand berührt, wird der Schluckreflex ausgelöst, das weitere Prozedere entzieht sich nun aktiven Interventionen. Die Dauer der oralen Phase wird von Böhme mit 1 - 1,5 Sekunden angegeben (Böhme 2003).

\subsubsection{Die pharyngeale Transportphase}

Passiert der Bolus den Pharynx in aborale Richtung, werden obere und untere Luftwege abgedichtet, um einen Übertritt der Nahrung in die Nase (nasale Regurgitation) bzw. in die Luftröhre (Verschlucken) zu verhindern (Foucart et al. 1998, Logemann 1988). Es handelt sich um die reflektorische Atmungsunterbrechung. Dazu wird das Velum eleviert und zum Verschluss der Trachea die Epiglottis nach dorsal abgesenkt, woran der Zungengrund beteiligt ist (Engelke et al. 1995b; Suto et al. 1995). Die Stimmlippen schließen, und die Mundbodenmuskulatur kontrahiert, der Larynx hebt sich (Logemann 1988) in Verbindung mit dem Hyoid, letzteres erreicht dabei seine höchste Position (Anagnostara et al. 2001), was palpatorisch nachvollzogen werden kann (Böhme 2003). Durch die Elevation des weichen Gaumens hin zur Pharynxwand erfolgt im Sinne des Biofunktionellen Modells nach Engelke 
eine Trennung der Kompartimente 3 und 4, hingegen treten BFK 2 und 3 in Kontakt miteinander; durch Aufhebung des linguovelaren Verschlusses wird die Boluspassage in den mittleren Pharynx möglich. Es schließt sich eine Welle der pharyngealen Peristaltik an, die der Pharynxentleerung sowie der Fortbewegung des Bolus dient (Logemann 1988). Dieser passiert den nun offenen, oberen Ösophagussphinkter (Böhme 2003). Die beschriebenen Abläufe der pharyngealen Phase nehmen maximal eine Sekunde Dauer in Anspruch (Böhme 2003).

In der späten pharyngealen Phase interagieren Pharynxwand und Gaumensegel auf unterschiedliche Weise so miteinander, dass dies Auswirkungen auf die potentielle Einnahme der Ruhelage hat. In Abhängigkeit davon, ob sich der Kontakt zwischen Velum und Pharynxwand löst, wird Kompartiment 2 im Übergang zu Kompartiment 3 dorsal geschlossen. Ist dies der Fall, so kommt es zum beschriebenen postdeglutorischen, physikalisch stabilisierten, geschlossenen, biofunktionellen Ruhezustand des orofazialen Systems.

\subsubsection{Die ösophageale Transportphase}

Der Eintritt des Bolus in die Speiseröhre wird durch Öffnung des oberen ÖsophagusSphinkters am Ende der pharyngealen Transportphase ermöglicht; willkürlich nicht beeinflussbar findet nun ein vegetativ-peristaltisch induzierter, ösophagealer Transport zum Magen statt (Böhme et al. 2006, Logemann 1988); die Atemwege öffnen wieder. Die Epiglottis weicht erneut nach kranial, der Hyoid-Larynx-Komplex senkt sich (Böhme 2003). Die Phase dauert bis zu 20 Sekunden (Böhme 2003).

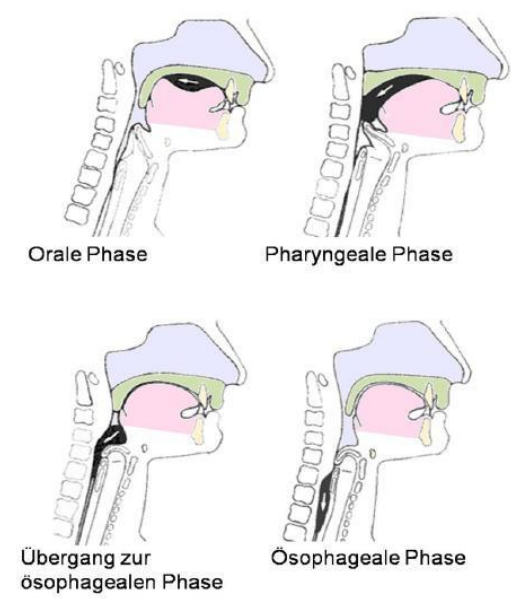

Abbildung 5 Phasen des physiologischen Schluckvorgangs (nach Logemann 1998) 


\subsection{Methoden zur Untersuchung der Saug- und Schluckfunktion}

Zur Beurteilung orofazialer Verhaltensmuster können klinische und rein visuelle Betrachtungen nicht immer genügen. Das oro-naso-pharyngeale Hohlraumsystem erfordert für eine genauere Diagnostik Methoden, die einen Erkenntnisgewinn in Gebieten erlauben, die von Weichteilen hermetisch verschlossen sind.

\subsubsection{Manometrie}

Die Manometrie ist ein physikalisches Verfahren, mit dessen Hilfe Druckmessungen in einem abgeschlossenen Raum durchgeführt werden. Das dazu benötigte Gerät ist ein Manometer. Der Druck gibt den Betrag einer auf einer Fläche A stehenden Kraft F an. Die SI-Einheit des Drucks ist Pascal $(\mathrm{Pa})$, daneben ist auch die Maßeinheit bar erlaubt. Es gilt: 1 bar $=1000$ mbar $=100000 \mathrm{~Pa}$. Medizinisch relevant ist auch die Druckangabe in MillimeterQuecksilbersäule (mmHg).

In der Allgemeinmedizin sind manometrische Messungen zur Druckbestimmung von Ösophagus und Darm verbreitet. Auf diese Weise können dort Funktionsstörungen nachgewiesen werden (Stein und Wehrmann 2006).

Tatsächlich trifft die Möglichkeit einer Funktionsdiagnostik mittels Manometrie - wie oben beschrieben - auch auf das orofaziale Organ zu. Intraorale manometrische Untersuchungen sind zahlreich vorhanden, allerdings muss in der existierenden Literatur unterschieden werden, an welchen Stellen innerhalb der Mundhöhle die Drücke gemessen wurden.

Im Grunde lassen sich zwei unterschiedliche Prinzipien feststellen: Einerseits kann die Druckmessung zwischen Festkörpern stattfinden, $\mathrm{zu}$ denen das anatomische Gewebe zu zählen ist. Dabei kommt es zu Messungen von Kontaktdrücken zwischen zwei (oder mehr) anatomischen Strukturen. Diese Messungen resultieren im Allgemeinen in positiven Druckwerten. Andererseits besteht die Möglichkeit, Drücke innerhalb von Fluiden wie Gasen, die sich in Hohlräumen befinden, zu messen. Hierbei erzielen die Messungen entweder positive oder negative Druckwerte.

Arbeiten zur intraoralen Messung von Kontaktdrücken können beispielsweise mithilfe eines Dehnungsmessstreifens, auf den die Drücke aktiv wirken, stattfinden (Howell und Manly 1948, Howell und Brudevold 1950, Winders 1956). Eine andere Möglichkeit der Messung 
positiver Drücke wählte 1992 eine Arbeitsgruppe um Robin (Robin et al. 1992), deren Arbeit in ihrer Weiterführung Einzug in die phonetische- und in die Schluckdiagnostik fand (Robbins et al. 1995, Lazarus et al. 2000, Vitorino 2010): Mittels eines in der Mundhöhle platzierten, pneumatisierten Bulbus wurde dabei der Druck der Zunge auf umliegende orale Strukturen gemessen. Auch White et al. nutzten 2009 die Manometrie für Messungen der Zungenkraft, die gegen den harten Gaumen ausgeübt wird.

Die intraorale Manometrie innerhalb von Funktionsräumen mit negativen Druckergebnissen hingegen stellte die Grundlage des biomechanischen Verständnisses des orofazialen Systems dar: Wichtige frühe Arbeiten auf Grundlage intrakompartimentärer Manometrie stammen von Faigenblum 1966, Fränkel 1967 und Krysinski und Piotrowski 1990. Fröhlich et al. (1991) wiesen neben Kontaktdrücken, die die Zunge gegen die Frontzähne ausübt, ebenfalls negative Drücke nach, die sie im subpalatinalen Mundhöhlenbereich maßen. Durch die Messung negativer Drücke innerhalb der Mundhöhle können die Theorien von Körbitz (1914) und Noltemeier (1949) bekräftigt werden, sodass der Begriff des Saugraumes manometrisch objektiviert wird. Andererseits fällt im Vergleich dieser Arbeiten auf, dass es zu Messdifferenzen zwischen Faigenblum, Fränkel und Krysinski und Piotrowski von etwa 3 $\mathrm{mmHg}$ bis $30 \mathrm{mmHg}$ an Unterdruck kommt. Eine weitere wichtige Arbeit im Zusammenhang mit manometrischer Untersuchung von Saugschluckvorgängen ist die Studie von Nilsson et al. aus dem Jahr 1996. Im sogenannten ROSS-Test (Repetitive Oral Suction Swallowing Test) wurden Probanden aufgefordert, über einen Strohhalm Wasser aus einem Becher zu trinken. Am Strohhalm wurden dabei subatmosphärische Drücke von weniger als -200 mbar gemessen. Der ROSS-Test stellt neben seinen manometrischen Ergebnissen eine wichtige methodische Grundlage für Schluckuntersuchungen in Bezug auf die Versuchsanordnung dar: das Ansaugen von Flüssigkeit wird zur Stimulation des Auslösens von Saug- und Schluckvorgängen gebraucht. Kieser et al. (2008) maßen an 5 Probanden, die $10 \mathrm{ml}$ Wasser schluckten, ebenfalls negative Drücke, die während funktioneller Aktivität auftraten. Die Arbeitsgruppe von Engelke et al. (2010) ordnete ihre Druckmessungen in den Rahmen des bereits erläuterten Biofunktionellen Modells ein: Dazu wurde der Druck sowohl im interokklusalen (BFK 1) als auch im subpalatinalen (BFK 2) Kompartiment gemessen. Die Vermutung war dabei, dass es weder grundlegende Druckunterschiede zwischen unterschiedlichen Funktionsphasen gebe, noch zwischen den Kompartimenten selber (Engelke et al. 2011). Manometrische Untersuchungen in beiden Kompartimenten an 20 Probanden bei zunächst offenem, dann halb-offenem Mund, daraufhin Messungen bei 
biofunktioneller Ruhelage und zuletzt beim Schlucken ergaben ein Druckmonitoring, das an dieser Stelle deshalb näher erläutert werden soll, weil es in seinen Grundresultaten für die manometrisch gestützte Diagnostik des Saugschluckvorgangs in der aktuellen Studie dienlich war: Es wurde gezeigt, dass während der Phasen mit offenem bzw. halb-offenem Mund beinahe identische, atmosphärische Drücke in beiden Kompartimenten vorlagen. Dies manifestiert sich im Druckdiagramm in einer Nulllinie, die einen Druckausgleich mit der Umgebung bedeutet. Während des Schluckens hingegen zeigen sich Peaks im Druckdiagramm, bei denen die absoluten Werte von BFK 1 und BFK 2 eindeutig voneinander abweichen. Im BFK 2 wurden fast durchgängig stärkere negative Drücke gemessen als im BFK 1. Dabei ist die Größe des Unterschiedes zwischen BFK 1 und 2 in Abhängigkeit von der jeweiligen Funktion verschieden. Während der postdeglutorischen, stabilisierten Ruhephase (z.B. nach ZRM) und beim Schlucken werden Drücke von -50 bis -150 mbar gemessen. Im Durchschnitt der gemessenen Drücke bestehen grundlegende Unterschiede zwischen den Phasen mit offenem Mund einerseits und den Phasen mit geschlossenem Mund, wie z.B. beim Schlucken, andererseits. Während der offenen und der halb-offenen Mundphase bilden sich keine Peaks, was auf eine nicht bestehende Kompartimentbildung zu diesem Zeitpunkt zurückgeführt wird. Beim Schlucken hingegen, sowie auch bei der geschlossenen Ruhelage nach Schluckakt, zeigen sich Peaks, die als geschlossene und in sich abgegrenzte Kompartimente interpretiert werden können.

Während der stabilisierten Ruhelage wird im BFK 2 ein Durchschnittswert von -81,86 mbar, im BFK 1 von -48,79 mbar gemessen. Engelke et al. (2011) begründen die höhere Generierung negativer Drücke im BFK 2 mit der Aktivität von Wandstrukturen, also Gaumen- und Zungenbewegungen. Das BFK 1 mit niedrigeren Drücken spiele dabei nur eine passive Rolle. Es werde nichtsdestotrotz eindeutig gezeigt, dass die beiden Kompartimente als jeweils selbstständige Einheiten zu sehen sind. Engelke et al. widerlegen damit ihre NullHypothesen. Überraschend sei auch die Abwesenheit positiver Druckwerte während aller Situationen im gesamten Versuch. Die genannten Autoren halten es dennoch für möglich, dass es zeitgleich zu negativen Drücken in den BFK und zu positiven Drücken zwischen Weichgewebe und Zähnen oder Gaumen kommen könne.

Mithilfe manometrischer Methoden konnte gezeigt werden, dass sich in beiden Kompartimenten subatmosphärische Drücke entwickeln und dass von einer unabhängigen Kompartimentbildung auszugehen ist. Diese Ergebnisse konnten durch intraoral bis in die jeweiligen Kompartimente eingeführte Schläuche gewonnen werden, die an ein 
manometrisches Messgerät angeschlossen sind. In methodischer Anlehnung an diese Arbeit erbrachte eine Manometrie im subpalatinalen Raum bei Santander et al. 2013 bei aktiver Bolusgabe eine mittlere Druckamplitude von etwa -290 mbar. Bei dieser Studie wurde die Abhängigkeit des Unterdrucks von der Boluskonsistenz geprüft.

Die Manometrie erweist sich als hilfreiche Methode, intraoral bestehende, physikalische Unterschiede bezüglich des Druckes zu erkennen und auf diese Weise die Funktionsräume näher zu charakterisieren. Sie dient auch dazu, eine Vorstellung von der Größenordnung der Drücke zu bekommen und diese miteinander ins Verhältnis zu setzen; damit wird die Manometrie ein wichtiger Teilbereich in der Methodik zur intraoralen Funktionsdiagnostik.

\subsubsection{Videofluoroskopie und Videokinematographie}

Neben der Manometrie sind bildgebende Verfahren in der medizinischen Diagnostik unverzichtbar. Unter sie fallen diverse apparative Untersuchungsmethoden, die zwei- oder dreidimensionale Bilddaten von Organen und Strukturen des menschlichen Körpers liefern und zur Diagnose krankheitsbedingter Veränderungen eingesetzt werden. Die Verfahren lassen sich nach Art der Erzeugung des Bildes einteilen. So arbeiten Videofluoroskopie und Videokinematographie mit Röntgenstrahlung, während die anderen Verfahren abweichende Techniken der Bilderzeugung nutzen. Die Bildgebung kann isoliert erfolgen, aber auch kombiniert mit beispielsweise akustischen, myographischen oder manometrischen Aufzeichnungen stattfinden.

Die Videofluoroskopie gilt seit langer Zeit als Standardverfahren in der Diagnostik des Schluckens (Rugiu 2007, Logemann et al. 1998). Mit der Einführung des endoskopischen Verfahrens in die Schluckdiagnostik durch Langmore et al. 1988 werde laut Rugiu (2007) die Videofluoroskopie allerdings in letzter Zeit ein wenig aus ihrer Schlüsselrolle verdrängt. In dem weit verbreiteten Verfahren wird bei dieser Technik ein Bolus unterschiedlicher Konsistenz, der zum Zwecke der Bildresonanz mit einem Kontrastmittel - meist Barium angereichert ist, vom Patienten geschluckt. Die dabei aufgenommenen Bilder dienen primär der Fokussierung auf den Bolus und entstehen radiologisch mit einem Röntgengerät. Die elektromagnetische Strahlung eines Röntgengerätes durchleuchtet das fokussierte Feld dergestalt, dass insbesondere dichte und feste Strukturen, wie z.B. Knochen, gut sichtbar gemacht werden. Die biologische Wirkung der Röntgenstrahlung ist hinreichend bekannt: Durch die ionisierende Strahlung können Veränderungen im lebenden Gewebe hervorgerufen 
werden. Bei entsprechender Strahlenexposition können kanzerogene Wirkungen nicht ausgeschlossen werden (Kauffmann et al. 2006). Wegen des in den Bolus eingemischten Kontrastmittels bestehen im Falle einer Aspiration die Risiken von Pneumonien sowie Fremdkörperreaktionen (Zhang et al. 2012).

Das unter anderem von Pokieser 2010 postulierte Verfahren der Videokinematographie ist analog zur Videofluoroskopie zu betrachten. Eine Durchleuchtungseinheit zeichnet die Deglutition des Patienten auf, während dieser ein Kontrastmittel schluckt. Die dabei videotechnisch dokumentierten Schluckvorgänge können zu diagnostischen Zwecken auch in Zeitlupe betrachtet werden, sodass eventuell vorhandene pathologische Schluckmuster erkannt werden können. Mit bis zu 25 Bildern / s ist eine gute Auflösung vorhanden (Böhme 2003). Die Videokinematographie kommt häufig dann zum Einsatz, wenn Funktionsstörungen angenommen werden, die ihren Ursprung in den oberen Speisewegen haben (Pokieser et al. 1995, Pokieser und Scharitzer 2004, Pokieser 2010).

\subsubsection{Endoskopie}

Endoskopie, die zur Bewertung des Schluckaktes herangezogen wird, wird als „Fiberoptic Endoscopic Evaluation of Swallowing (FEES)“ bezeichnet (Langmore et al. 1988, Hiss und Postma 2003, Böhme 2003). Sie ergänzt und ersetzt teilweise die Videofluoroskopie (Rugiu 2007, Nacci et al. 2008) und kommt in der Schluckdiagnostik routinemäßig zum Einsatz (Schröter-Morasch et al. 1999). Mithilfe eines flexiblen Endoskops, das transnasal bis zum Pharynx eingeführt wird, lässt sich der Schluckvorgang auf einem Monitor beobachten. Ebenfalls kann für die rein strukturelle Begutachtung auch die transorale, starre Applikationstechnik mit $90^{\circ}$ Winkeloptik (TOES) sowie bei Patienten mit Tracheostoma eine transstomatale Technik Anwendung finden. Für den Untersucher besteht bei Aufzeichnung des Bildmaterials die Möglichkeit, nachträgliche Aufbereitungen und Bildoptimierungen durchzuführen, die die Diagnostik vereinfachen können. Weitere Vorteile dieses Verfahrens sind die im Vergleich zu anderen Techniken niedrigen Anwenderkosten sowie die Tatsache, dass es zu keiner Exposition schädlicher Strahlung kommt. Zudem ist die Endoskopie nicht ortgebunden und kann direkt am Krankenbett verfügbar gemacht werden. Als Nachteil des Verfahrens wird angeführt, dass dem Untersuchenden zum exakten Zeitpunkt des Schluckens die Sicht genommen werden kann, und sich damit möglicherweise wichtige, intradeglutitive Aspekte der Beobachtung entziehen. So sind die orale und auch die pharyngeale Phase nur 
eingeschränkt zu beurteilen (Logemann et al. 1998, Böhme 2003). Einige anatomische Strukturen sind darüber hinaus nicht oder nur bedingt quantifizierbar, wie beispielsweise der Mundboden, der kaum abzubilden ist. Es kann in einzelnen Fällen außerdem zu gesteigertem Würgereiz, zu vagalen Reaktionen oder auch zu Epistaxis kommen (Böhme 2003, Nacci et al. 2008).

\subsubsection{Sonographie}

Auch mittels der Sonographie (Ultraschall) kann medizinische Diagnostik organischen Gewebes betrieben werden. Ein wesentlicher Unterschied im Vergleich zur Röntgenstrahlung liegt in der Unschädlichkeit der angewendeten Strahlung. Sonographie wird in erster Linie am Herzen, an der Schilddrüse, an den Harnwegen und der Harnblase, aber auch zur Beurteilung von Gefäßen angewendet. Sonographische Untersuchungen zum Schluckakt fanden bereits 1988 durch Wein et al. statt, die die postdeglutitive fixierte Zungenanlagerung an den harten Gaumen auf diesem Wege bestätigten. An Säuglingen testeten Wein et al. 1993 mit einer kombiniert sonographisch-manometrischen Studie die Darstellbarkeit der Zunge beim Saugvorgang mit Ultraschall, sie können dabei unter anderem eine dorsokraniale Retraktion der Zunge während des Sogaufbaus feststellen. Die simultane intraorale Druckmessung zusammen mit bildgebender Sonographie erachten sie in der Diagnostik des Saugens als positiv. 2004 untersuchten Peng et al. insgesamt 55 Patienten mit teils somatischem und teils viszeralem Schluckmuster per Ultraschall und stellten Unterschiede in der Dauer der einzelnen Schluckphasen fest. Weitere Studien fanden unter anderem durch Neuschäfer-Rube et al. statt, die 1997 gesunde sowie dysphagische Patienten verschiedener Ätiologie sonographisch untersuchten. Die Bildgebung erfolgt bei der Ultraschalltechnik nach dem sogenannten Echo-Impuls-Verfahren. Ultraschallimpulse werden von einem Schallkopf aus in das Gewebe eingekoppelt, die ausgesendeten Schallwellen werden je nach Gewebe, auf das sie auftreffen, unterschiedlich gestreut, absorbiert und / oder reflektiert. Durch ein zurück, in Richtung Schallkopf gerichtetes Schallecho kann der Sonograph ein zweidimensionales Schnittbild des untersuchten Gewebes errechnen. Die Technik erlaubt bedside-Anwendung, stellt - wie beschrieben - keine Strahlenbelastung dar und ist beliebig oft durchführbar (Böhme 2003). Ein limitierender Faktor im Anwendungsbereich der Sonographie ist die von der Frequenz der ausgestrahlten Impulse abhängige Eindringtiefe der Schallwellen (Böhme 2003), ein Nachteil, der im Zusammenhang des Schluckvorgangs insbesondere bei der 
Darstellung der pharyngealen, aber auch der oralen Phase zum Tragen kommt (Akin et al. 2006). Denn je tiefer das anzuschauende Gebiet liegt, desto schwächer wird das Auflösungsvermögen. Gerade an profund liegenden Gebieten wird die Qualität der Bildgebung eindeutig von derjenigen der Computertomographie (Röntgen) und Magnetresonanztomographie übertroffen. Knochen und luftgefüllte Hohlräume stellen für die Ausbreitung der Schallwellen beinahe unüberwindbare Barrieren dar (Grau 2009), da es zu Schallschatten kommen kann. Artefakte sind keine Ausnahme und gehen mit der Anwendung fix einher (Grau 2009). Die Dokumentation sonographischer Untersuchungen ist im Vergleich $\mathrm{zu}$ anderen bildgebenden Verfahren schwierig, da es keine Standardisierungen geben kann. Schnittebenen sind schlecht definierbar. Die Bildgebung ist sehr von der individuellen Sonden- und damit Impulsführung des Untersuchenden abhängig und daher nicht reproduzierbar. Aufgrund der aufgeführten Faktoren hat das sonographische Verfahren für die Diagnostik des Schluckens kaum klinische Relevanz erlangen können, die Darstellung des Schluckakts im oro-pharyngo-laryngealen Bereich ist mit dieser Technik nur eingeschränkt möglich (Chi-Fishman 2005).

\subsubsection{Magnetresonanztomographie}

Die Magnetresonanztomographie, auch Kernspintomographie oder kurz MRT (MRI, NMR) genannt, wird in der medizinischen Diagnostik eingesetzt, um Lage, Form, Struktur und auch Funktion von Gewebe und Organen in allen drei Körperebenen darzustellen. Diese Aufgabe übernimmt ein Magnetresonanztomograph, der Schnittbilder des menschlichen Körpers erzeugt, mittels derer eine Beurteilung von Organen und krankhaften Organveränderungen stattfinden kann. Das dem zugrunde liegende Verfahren beruht auf starken Magnet- und elektromagnetischen Wechselfeldern, in denen der Körper (bzw. Teilbereiche des Körpers) sich befindet. Dadurch werden vor allem Wasserstoffkerne des Gewebes resonant angeregt, was zu elektrischen Signalen im Empfänger führt, die digitalisiert und in ein Bild umgerechnet werden (Reiser und Semmler 2002). Wichtig, und damit ebenfalls abzugrenzen von röntgenologischen Verfahrenstechniken, ist auch hier die Unschädlichkeit für den menschlichen Körper. Nach heutigem Kenntnisstand bestehen keinerlei Risiken für Organschädigungen o.Ä. bei Einhaltung der empfohlenen Grenzwerte, da keine belastende Röntgenstrahlung oder andere ionisierende Strahlung erzeugt wird (Vogl 1991). Die abgebildeten Gewebe erscheinen je nach Dichte in charakteristischer Intensitätsverteilung von Grauwerten. Die Qualität der Bildgebung ist maßgeblich von der Magnetfeldstärke abhängig, 
neben dieser aber auch von anderen Parametern. Im Vergleich $\mathrm{zu}$ anderen bildgebenden Verfahren lässt sich bei der MRT oft eine verbesserte Darstellung vieler Organe erzielen (Pasler 1989), was insbesondere die Weichgewebe betrifft, von denen verschiedene Signalintensitäten ausgehen. Neuronales Gewebe wie z.B. das Gehirn wird erst im MRT darstellbar. Es kann eine sehr hohe Detailgenauigkeit erreicht werden, die bei röntgenologischen Techniken nicht zu erzielen ist. Allerdings können wegen des starken Magnetfeldes Metalle, die sich am oder im Körper befinden, Nebenwirkungen und Bildstörungen verursachen. Es kann zu Verlagerungen und Erwärmungen während der Untersuchung kommen. Des Weiteren kann die MRT-Diagnostik im Vergleich zu anderen Verfahren durchaus zeitaufwändiger sein. Hartgewebe wie knöcherne Strukturen sind u.U. schlechter darzustellen als in der Röntgendiagnostik, da sie wenig Wasser und wenig Fett enthalten, deren Resonanz im MRT zu einer besonders detailgetreuen Darstellung genutzt wird. Die Patienten werden in einer Röhre relativ geringen Durchmessers (etwa $60 \mathrm{~cm}$ ) untersucht. Dies kann zu Angst- und Beklemmunsgefühlen führen. Während der Aufnahmen kommt es zu lauten Geräuschen.

Die Unschädlichkeit und Non-Invasivität des Verfahrens sowie die gute Weichgewebsdarstellung ließen die MRT in den vergangenen Jahren zu einem zunehmend bevorzugten Verfahren auch im Bereich der Diagnostik orofazialer Weichgewebe werden. Die dazu in der Literatur vorhandenen Untersuchungen unterscheiden sich allerdings hinsichtlich der geprüften Parameter und deren qualitativer Darstellbarkeit, auch in Abhängigkeit technischer Einstellungen. Wichtige Strukturen wie z.B. die Zunge untersuchten Niitsu et al. bereits 1994 per Magnetresonanztomographie. Sie beobachteten die Zungenbewegungen dreier Probanden während verschiedener phonetischer Aktivitäten. Ein Jahr später führten Suto et al. 1995 Schluckuntersuchungen per MRT durch, bei denen sie den Bolus mit einem Kontrastmittel anreicherten und seine Propulsion von der Mundhöhle in den Ösophagus verfolgen konnten. Sie beobachteten die Zunge, den weichen Gaumen, die hintere Pharynxwand sowie das Hyoid und die Epiglottis beim Schlucken. Eine Arbeitsgruppe um Anagnostara (2001) verglich einige Jahre später in einer Schluckuntersuchung sieben gesunde Probanden an drei verschiedenen, zeitlich hochauflösenden MRT-Systemen. Zu den beobachteten Strukturen zählten dabei die Zunge, das Velum, die Pharynxwände sowie Epiglottis und Hyoid, die allerdings häufig durch Artefakte verdeckt wurden. Von Bewegungsartefakten, insbesondere während des Kauvorgangs, ist auch bei Hartl et al. (2003) die Rede. In dieser Arbeit wurden ebenfalls sieben gesunde Probanden untersucht, die Stoffe verschiedener Konsistenz 
schluckten. Sie stellten eine sehr unterschiedliche Visualisierungsmöglichkeit der verschiedenen anatomischen Parameter während des Schluckens fest. Ohkubo et al. definierten 2008 die Lippen, die Spitze, den Körper und die Basis der Zunge, Velum und Epiglottis als anatomische Marker, die als wichtig in der Schluckdiagnostik in Erscheinung treten. An fünf gesunden Probanden evaluierten sie Unterschiede in der Darstellung dieser Parameter zwischen drei unterschiedlichen Modalitäten in der Bildgebung. In einer weiteren 2009 veröffentlichten Studie von Breyer et al. werden der Oropharnyx und der Larynx während Atmung, Zungenbewegungen und Schlucken durch ein MRT mit 3 Tesla visualisiert. Panebianco et al. (2010) wählten in einer Studie an 23 gesunden Probanden die Parameter Zunge, Velum, Pharynx, Epiglottis und Larynx-Hyoidknochen. Die Magnetresonanztomographie wird dabei als effektiv und hilfreich in der Bewertung von Schluckbewegungsmustern angesehen.

Einige der zitierten Studien zum Schluckakt mit MRT-Diagnostik resultieren in der Feststellung, dass die zeitliche Auflösung im konventionellen MRT-Verfahren nicht ausreiche, um chronologische Aussagen hinsichtlich der Dauer einzelner Bewegungsparameter zu treffen (Anagnostara et al. 2001, Hartl et al. 2003, Panebianco et al. 2010). So könne beispielsweise die Boluspassage aufgrund einer ungenügenden Zeitauflösung nicht im Einzelnen präzise bemessen werden (Panebianco et al. 2010). Zudem sei technikbedingt auch aufgrund einer Lateralisation des Bolus dessen Visualisierung im medio-sagittalen Schnittbild nicht lückenlos nachvollziehbar (Hartl et al. 2003). Panebianco et al. benutzten dabei 2010 eine Bildfrequenz von 3-4 Bildern / s, womit es ihm nicht gelang, in jedem Falle eine gute Bildgebung der kurzen, pharyngealen Phase zu erhalten. Hartl nutzte 2003 eine zeitliche Auflösung mit Bildgebung nach jeweils 700 ms, womit er ein oder zwei Bilder des etwa 1,5 s dauernden, eigentlichen oropharyngealen Schluckakts erhielt. In einer kürzlich durchgeführten Untersuchung erreichten Zhang et al. (2012) mit einer Bildfrequenz von fast 25 Bildern / s eine Schluckaktwiedergabe in Echtzeit, vergleichbar mit der Auflösung der Videofluoroskopie. An 10 Probanden testeten sie mit Ananassaft, der mit Stärkepulver angedickt war, Aufnahmen des Schluckvorgangs mithilfe eines 3-Tesla-Tomographen. Sie loben eine Darstellung in hoher Qualität und zudem in drei körperlichen Ebenen, kommen aber zu dem Schluss, dass die sagittale in den allermeisten Fällen die optimale Achse zur Beurteilung der am Schlucken beteiligten Strukturen darstelle.

Im Vergleich der Schluckuntersuchungen im MRT unterscheiden sich des Weiteren die Meinungen über die Effizienz einer Bolusanreicherung mit einem Kontrastmittel: Während 
dies in einigen Studien mit dem Ziel einer visuellen Verfolgung des Bolus empfohlen wird (Anagnostara et al. 2001), ist andererseits von einer guten Resonanz von Wasser die Rede, das per se in der Bildgebung zu Signalen höherer Intensität führe und sich demnach heller darstelle als Stoffe soliderer Konsistenz (Hartl et al. 2003).

Eine weitere in der Literatur vorzufindende Fragestellung ist diejenige nach einem Einfluss der liegenden Position der Probanden auf die Ausführung eines physiologischen Schluckakts. Studien, die eine horizontale Position für die Schluckdiagnostik für untauglich halten (Suto et al. 1995, Foucart et al. 1998) stehen denjenigen gegenüber, die keine Einflussnahme einer nicht-aufrechten Position auf das physiologische Schlucken feststellen konnten und dieser widersprechen (Zhang et al 2012).

Neben der Kritik an der liegenden Position im MRT beim Schlucken ist bei Suto et al. (1995) zudem von eingeschränkten, weil verzögerten Interventionsmöglichkeiten beim Auftreten von Aspirationen die Rede, was insbesondere bei dysphagischen Patienten zu beachten sei. Auch Anagnostara et al. (2001) erwähnen erschwerte Detektionsmöglichkeiten von Penetrationen und Aspirationen.

Die Magnetresonanztomographie wird im Rahmen der Schluckdiagnostik zunehmend häufig eingesetzt. Hartl et al. (2003) sehen die Magnetresonanztomographie zwar heutzutage noch ergänzend zur Videofluoroskopie, erkennen in ihr aber durchaus das Potenzial, diese eines Tages zu ersetzen, etwa deshalb, da eine bessere Bewertung tiefer gelegener Strukturen, deren Volumina und deren Mobilität gegeben sei.

\subsubsection{Manofluorographie und Manovideoendoskopie}

Die Kombination aus Pharynxmanometrie und Fluoroskopie, die in der Literatur als Manofluorographie beschrieben ist (McConnel et al. 1988), ermöglicht die Beobachtung und Beschreibung von Zusammenhängen zwischen dem visualisierten Schluckakt bzw. der Boluspassage und simultan gemessenen Drücken. Die Manofluorographie stellt die einzige Methode dar, den während der Pharynxpassage auf den Bolus ausgeübten Druck (Intrabolusdruck) zu messen, da hierzu eine zeitgleiche Visualisierung notwendig ist. Zudem kann bei dieser Technik die Positionierung der Drucksensoren visuell überprüft werden.

Letzteres ist auch bei der von Butler et al. 2009 beschriebenen Manovideoendoskopie, der Kombination aus pharyngealer Manometrie und Endoskopie, möglich. Sie bietet Vorteile 
gegenüber der Manofluorographie in Hinsicht auf Transportierbarkeit und bedsideBehandlung, da die Patienten keine radiologische Untersuchung benötigen.

\subsection{Fragestellungen und Zielsetzung der Arbeit}

Ziel unserer Untersuchung ist es, die funktionellen Verlagerungen der anatomischen Strukturen während Saugschluckvorgängen $\mathrm{zu}$ prüfen. Aufgrund vorliegender Untersuchungen muss von einer Expansion des subpalatinalen Raumes während des Saugvorganges ausgegangen werden. Dabei ist jedoch ungeklärt, auf welche Weise diese Erweiterung stattfindet und welchen Anteil einzelne Biofunktionelle Einheiten an der Entstehung dieses Raumes haben.

Mithilfe magnetresonanztomographischer Darstellungen können zyklisch ablaufende Saugschluckvorgänge in der Mediosagittalebene visualisiert werden und Aufschluss über die Verlagerung anatomischer Strukturen geben. Über eine manometrische Untersuchung des subpalatinalen und des interokklusalen Raumes können die Zyklen aus Saugen und Schlucken identifiziert werden.

Ziel der Arbeit ist es, in einem diagnostischen Setting mit Manometrie und MRT die Verlagerung der einzelnen Strukturen während der Saugschluckvorgänge näher zu definieren. Geprüft werden sollen in Abhängigkeit von einzelnen Phasen des Saugschluckvorgangs:

1.) Die Positionsänderung des Unterkiefers relativ zum Oberkiefer

2.) Die Dynamik des subpalatinalen Kompartiments

3.) Veränderungen der dorsalen Zungenbegrenzung

4.) Veränderungen der dorsalen Velumkontur

5.) Veränderungen der Position des Mundbodens

Die Resultate der Untersuchung sollen das Verständnis physiologischer Abläufe orofazialer Funktionen erweitern und als Grundlage für die Entwicklung neuer therapeutischer Ansätze in der Behandlung dysphagischer Patienten dienen. 


\section{Material und Methode}

\subsection{Probandenkollektiv}

Das Probandenkollektiv setzte sich aus 9 gesunden, erwachsenen Probanden zusammen, die sich freiwillig - nach vorheriger Aufklärung über den Studienablauf - zur Verfügung stellten. Ihr Alter lag zwischen dem 18. und 31. Lebensjahr, das Durchschnittsalter betrug 24,1 Jahre. Es wurden 4 Frauen und 5 Männer untersucht.

Zur Durchführung der vorliegenden Studie wurde an die Ethik-Kommission der Medizinischen Fakultät der Georg-August-Universität Göttingen ein Antrag gestellt (12/11/09). Nach positiver Begutachtung und daraufhin erteilter Genehmigung des Antrags konnte die Studie ohne ethische und rechtliche Bedenken durchgeführt werden. Das zum gestellten Ethikantrag ergangene Gutachten befindet sich im Anhang dieser Arbeit.

\subsubsection{Auswahl der Probanden}

Auf die Möglichkeit der Teilnahme an der Studie wurde durch Aushänge im Zentrum ZMK der UMG hingewiesen. Zur Teilnahme einbezogen wurden gesunde Probanden ohne Hinweise auf allgemeinmedizinische Risikofaktoren. Bei den Probanden handelt es sich um freiwillige Probanden beiderlei Geschlechts mit vollständiger Dentition, physiologischem Schluckmuster und Klasse I Verzahnung (Neutralbisslage).

Im Rahmen der Probandenauswahl wurde eine Anamnese erhoben, die anhand des im Anhang beigefügten „MRT / Manometrie-Studie; Erfassungsbogen“ nachvollzogen werden kann. Des Weiteren erfolgte die Aufnahme eines intraoralen Befundes durch einen der Prüfärzte. Eine Dokumentation über die Erfassung der erhobenen Befunde sowie über die weiteren im Rahmen der Studie durchgeführten Maßnahmen fand mittels eines Prüfbogens statt, der für jeden der Probanden ausgefüllt wurde. Auch er liegt dieser Arbeit im Anhang bei. 


\subsubsection{Aufklärung der Probanden}

Die an der Studie teilnehmenden Probanden wurden im Vorfeld der Untersuchung sowohl mündlich als auch schriftlich über Ziele, Art und Durchführung der Versuche aufgeklärt. Dies erfolgte durch ein Gespräch mit einem der Prüfärzte und auf schriftlichem Wege durch ausgehändigtes Informationsmaterial. Die Probanden bekundeten auf einer Einverständniserklärung per Unterschrift, umfassend und genügend aufgeklärt worden zu sein. Zudem bestätigten die Probanden mit ihrer Unterschrift die Zurkenntnisnahme gesonderter Aufklärungsbögen für die MRT-Untersuchung. Sie willigten - ebenfalls schriftlich - ein, die an ihnen ermittelten Daten in anonymisierter Form $\mathrm{zu}$ wissenschaftlichen Zwecken freizugeben. Der unterschriebene Aufklärungsbogen und die Einverständniserklärung galten als Voraussetzung zur Teilnahme.

\subsection{Messinstrumente und Geräte}

\subsubsection{Intraoraler Anteil des Applikations- und Messsystems}

Zur intraoralen Manometrie diente ein in die Mundhöhle eingesetztes Gerät, das eine Modifikation der in Kapitel 1.2.1 im Rahmen des ZRM vorgestellten Membrantrichterplatte (MTP; Silencos®, Bredent; Senden, Deutschland) darstellt. Es besteht aus einer den Wangen innen anliegenden, elastischen Mundvorhofplatte, die beidseits im Seitenzahnbereich endet und individuell flexibel adaptierbar ist. Das Lippenstück wird zwischen den Lippen platziert und umfasst drei Schlauchverbindungen zwischen intra- und extraoral, die durch Bohrungen innerhalb des Lippenstücks verlaufen.

Die erste Schlauchverbindung (V1) ist invers gerichtet und stellt eine Boluszufuhr in das subpalatinale Kompartiment (BFK 2) dar. Benutzt wurde ein PVC-Schlauch mit einem Innendurchmesser von $2 \mathrm{~mm}$. Der Schlauch bildet auf Höhe der ersten oberen Molaren eine Schlaufe. In diesem Bereich ist der Schlauch perforiert, damit der Bolus aus dem Schlauch ins BFK 2 austreten kann. Am Scheitelpunkt der Schlaufe ist das interne Lumen des Schlauches mit Kunststoff verschlossen (Relino Softæ, Kanidenta, Herford, Deutschland). 
Die zweite Schlauchverbindung (V2) stellt den austretenden Teil der Schlaufe dar und dient der Druckmessung im subpalatinalen Kompartiment (BFK 2). Hierzu ist hinter dem Scheitelpunkt der Schlaufe und dem dortigen Lumenverschluss erneut eine Perforation vorgenommen worden, die den Zugang für die Druckmessung im BFK 2 erlaubt. Der Schlauch mit 2 mm Innendurchmesser führt durch das Lippenstück hindurch nach extraoral.

Über die dritte Schlauchverbindung (V3) findet die Druckmessung im interokklusalen Kompartiment (BFK 1) statt. Hier mündet der Schlauch mit dem Endstück eines aus der zahnmedizinischen Praxis bekannten Speichelsaugers (saliva ejectors, orbis dental; Münster, Deutschland), dessen Draht entfernt wurde. Seine Öffnung liegt im linken vestibulum oris. Er ist an einen PVC-Schlauch (2 mm Innendurchmesser) angeschlossen, der durch das Lippenstück hindurch nach extraoral führt.

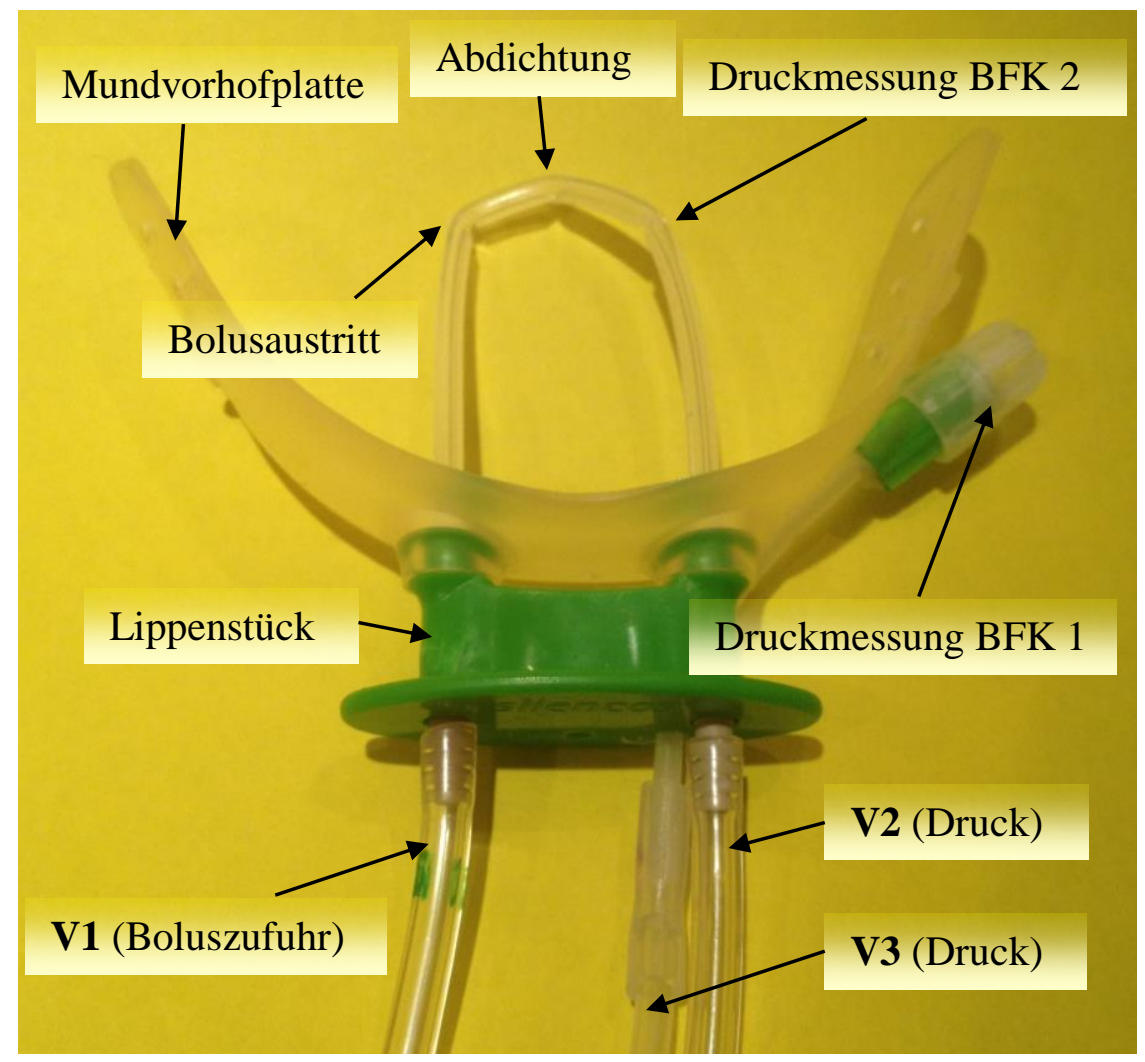

Abbildung 6 Modifizierte Membrantrichterplatte mit 3 Schlauchverbindungen zur Bolusapplikation und Druckmessung 


\subsubsection{Extraoraler Anteil des Applikations- und Messsystems}

Außerhalb des Mundes befinden sich folgende Schläuche:

Verbindung 1 (V1) sorgt über einen $150 \mathrm{~cm}$ langen Schlauch mit $2 \mathrm{~mm}$ Innendurchmesser für die Boluszuführung und ist an eine 50-ml-Spritze der Firma B. Braun ${ }^{\circledR}$ angeschlossen (Omnifix ${ }^{\circledR}$, B. Braun ${ }^{\circledR}$; Melsungen, Deutschland), die während des Ablaufes auf dem Bauch des Probanden liegt. Sie stellt das Reservoir für die Bolusapplikation dar.

Als Bolus dient dabei stilles, kohlensäurefreies Mineralwasser der Firma Vittel (Nestlé Waters; Mainz, Deutschland), das vor Beginn der Versuchsdurchführung in die Spritze gegeben wurde.

Verbindungen 2 und 3 (V2 und V3) führen aus dem subpalatinalen Kompartiment (BFK 2) bzw. dem interokklusalen Kompartiment (BFK 1) durch das Lippenstück nach extraoral und sind dort jeweils über Sensoren an ein Druckmessgerät angeschlossen. Zwischengeschaltet ist nach $5 \mathrm{~cm}$ ein Abscheidebehälter und ein Bakterienfilter (Aqua-Knot II Water Trap®; GE Medical Systems, Wisconsin, USA) (Draeger Medical Systems, Wisconsin, USA). Hier werden Flüssigkeiten gesammelt, die ins austretende Schlauchsystem gelangt sind. Die Behälter sind druckdicht von der Umgebung abgeschlossen.

Sowohl Verbindung 2 als auch Verbindung 3 führen über $5 \mathrm{~m}$ lange Schläuche mit $2 \mathrm{~mm}$ Innendurchmesser zu dem Drucksensor, der am Manometer angeschlossen ist.

Bei allen Schläuchen handelt es sich um PVC-Materialien der Fresenius Kabi® GmbH, Bad Homburg, Deutschland. 


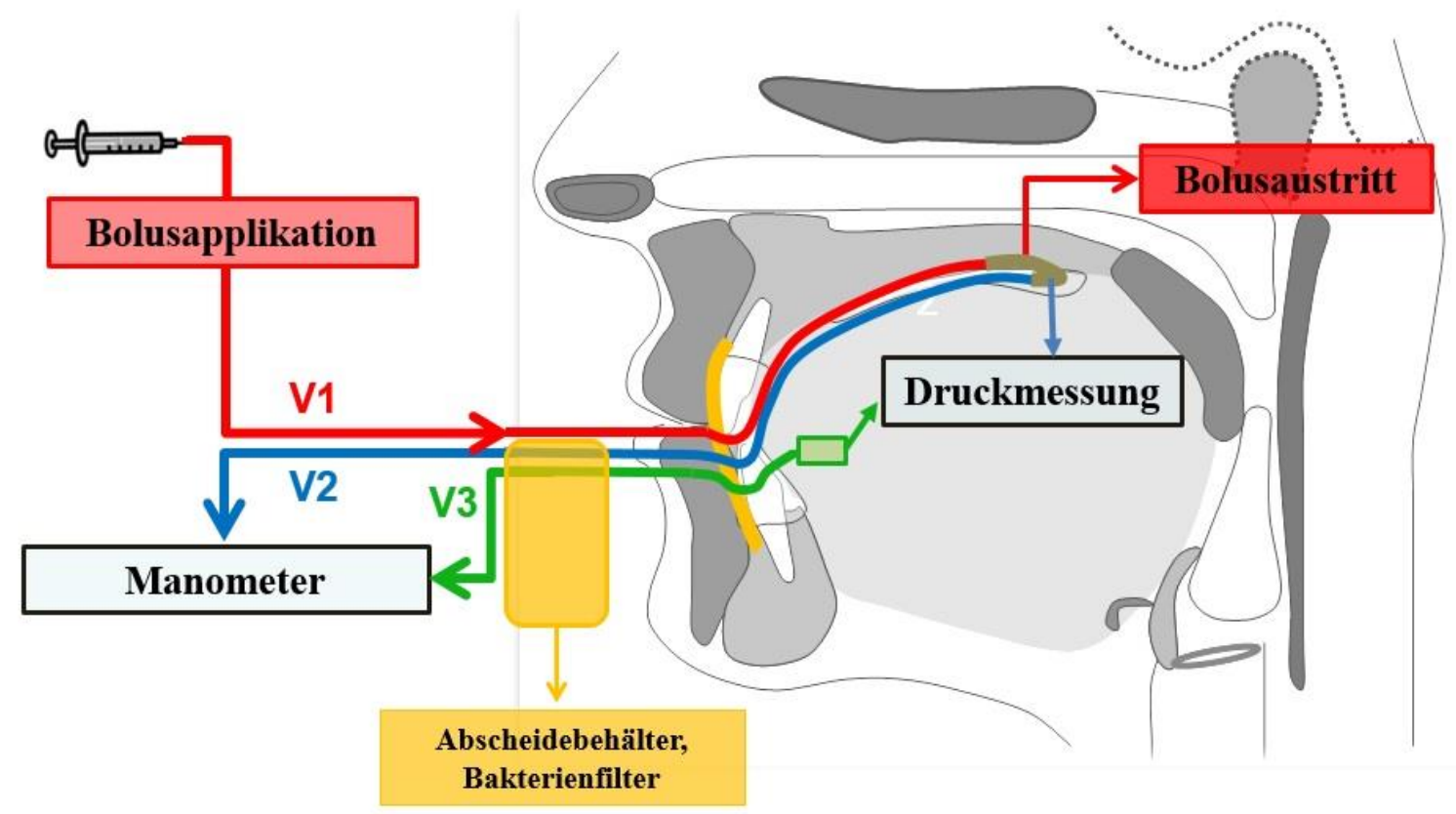

Abbildung 7 Schematische Darstellung des intra- und extraoralen Applikations- und Messsystems

\subsubsection{Räumlicher Aufbau des Messsystems}

Die Skizze verdeutlicht den Aufbau des Messsystems innerhalb der Räumlichkeiten, in denen die Untersuchung stattfand.

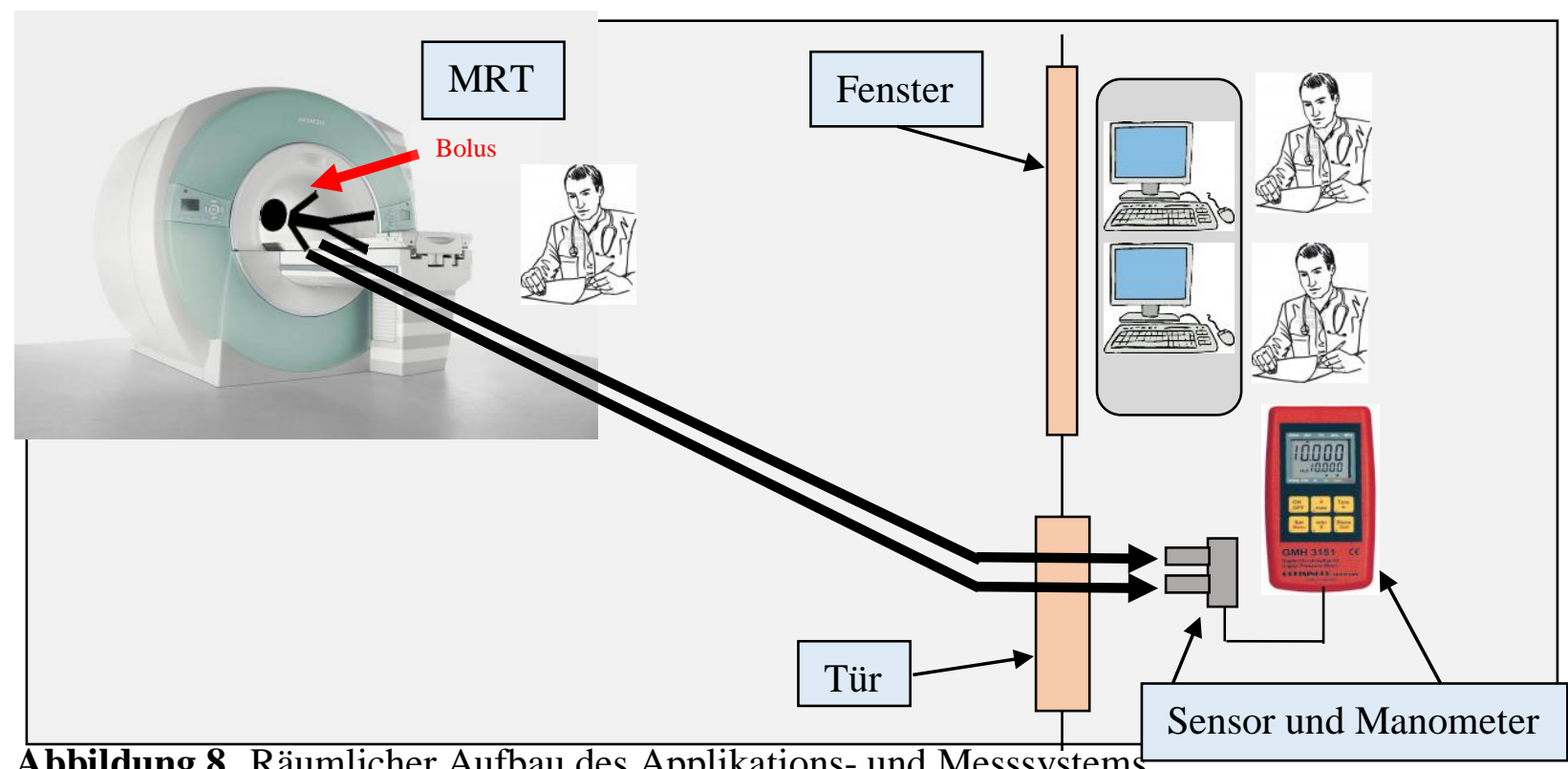




\subsubsection{Manometer}

Bei dem Druckmesssystem handelt es sich um ein CE-zugelassenes Handdruckmessgerät (GMH 3156, Greisinger electronics; Regenstauf, Deutschland), dessen Sensoren (GMSD 350 MR, Greisinger electronics; Regenstauf, Deutschland; Messbereich: $-199,9$ mbar bis $+350,0$ mbar) über PVC-Schläuche mit dem Mundstück verbunden sind, um den intraoralen Druck in den beiden Kompartimenten aufzuzeichnen. Es hat eine Messfrequenz von $1 \mathrm{kHz}$. Die Auswertung und spätere Bearbeitung der Daten erfolgte mit GSOFT3050 V3.0® (Greisinger electronics; Regenstauf, Deutschland).

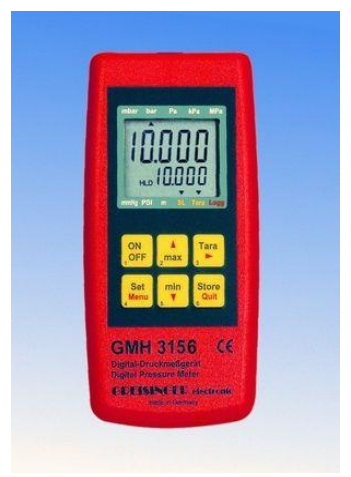

Abbildung 9 Handdruckmessgerät GMH 3156, Greisinger electronics; Regenstauf, Deutschland

\subsubsection{Magnetresonanztomograph}

Die Bildgebung erfolgte an einem 3-Tesla-MRT-Scanner (Magnetom Tim Trio; Siemens Medical Solutions, Erlangen, Deutschland). Die Bedienung des Tomographen wurde ausschließlich von dem entsprechend damit vertrauten Mitarbeiter der Abteilung Neuroradiologie der UMG (M.-N. P.) vorgenommen. Nach den obligatorischen Planungsaufnahmen (Localizer) wurde eine T1-Sequenz in koronarer Schichtführung mit 20 Schichten und eine Schichtdicke von $5 \mathrm{~mm}$ durchgeführt. Diese erste Aufnahme diente dazu, die folgenden Sequenzen in einer exakt mediosagittalen Ebene $\mathrm{zu}$ positionieren. Die Repetitionszeit betrug $611 \mathrm{~ms}$, die Echozeit $11 \mathrm{~ms}$, der Flipwinkel $120^{\circ}$ und die Aufnahmen zeigten eine Matrix von 256 x 256 Pixeln. Die zweite Aufnahme, die während des Schluckversuches durchgehend wiederholt wurde, war eine exakt sagittale T2-Aufnahme mit 
einer Schichtdicke von 5 mm und einer Matrix von 256 x 256 Pixeln. Das Zentrum des Field of View wurde im Bereich des Zungengrundes gewählt. Die Repetitionszeit betrug 54,88 ms, die Echozeit 1,69 ms, die zeitliche Auflösung 0,53 s und der Flipwinkel 50. Die Aufnahmen wurden zeitgleich mit dem Schluckakt begonnen und insgesamt 350 Mal wiederholt, um den gesamten Versuch bildgebend zu dokumentieren.

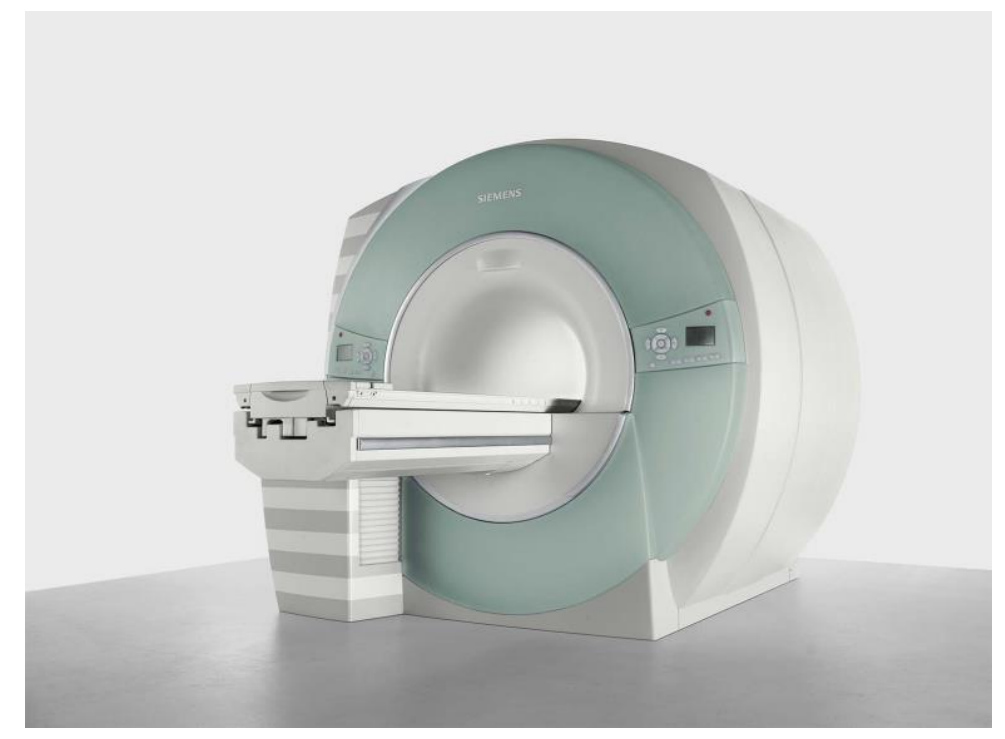

Abbildung 10 Magnetresonanztomograph Magnetom Tim Trio, Siemens Medical Solutions, Erlangen, Deutschland

\subsection{Durchführung der Untersuchung}

Vor der Versuchsdurchführung wurde diese in einer Übungsphase mit den Probanden außerhalb des MRT, aber ansonsten identischen Bedingungen, trainiert. Die Probanden konnten sich dadurch an Versuchsaufbau und -durchführung gewöhnen. Es bestand die Möglichkeit, die Probanden hinsichtlich möglicherweise auftretender Aspirationsprobleme, Akzeptanz des intraoralen Gerätes und Kooperationsbereitschaft zu beurteilen. Der Versuchsdurchführende bekam einen Eindruck über jeden Probanden bezüglich seiner Tauglichkeit für die Studie. 
Die Durchführung des Versuches fand in den Untersuchungsräumen der Abteilung für Neuroradiologie der Universitätsmedizin Göttingen statt. Der Proband liegt dabei im MRT und trägt das beschriebene Mundstück.

Er ist dazu aufgefordert, Wasser aus dem Reservoir anzusaugen, dieses herunter zu schlucken und anschließend den Mund zu öffnen. Diese Sequenz wird kontinuierlich wiederholt.

Der zyklisch wiederkehrende Vorgang aus Saugen und Schlucken wird als Saugschluckzyklus (SSZ) definiert. Die Saugschluckzyklen sind durch die experimentell eingeführte, intermittierende Mundöffnung voneinander abgegrenzt.

Die Abfolge aus Saugen, Schlucken und Öffnen des Mundes soll in etwa 5-10 Sekunden vonstattengehen, wobei die Rhythmusvorgabe dem Probanden durch den vorab durchgeführten Übungsdurchlauf geläufig war. Insgesamt dauert die Untersuchung 180 Sekunden.

\subsection{Analyse der erhobenen Daten}

Von jedem Probanden wurden drei Saugschluckzyklen (SSZ) zur Untersuchung ausgewählt: Analysiert wurden diejenigen, die nach 40 (SSZ 1), nach 80 (SSZ 2) und nach 120 (SSZ 3) Sekunden stattfanden. Diese Zyklen wurden mittels der Manometrie identifiziert und auf Gleichmäßigkeit der Druckverläufe überprüft. Daraufhin wurden die Zyklen zeitlich den zugehörigen MRT-Bildsequenzen zugeordnet. Die Bewegungsanalyse beteiligter Strukturen erfolgte auf Basis dieser Bildsequenzen.

\subsubsection{Manometrie}

Nach der Versuchsdurchführung wurden die ermittelten Druckdaten auf einen Computer übertragen, auf dem mit der Software GSOFT3050 die Datensätze in eine Grafik überführt wurden. Von den pro Sekunde ermittelten 1000 Messungen (1 kHz Messfrequenz) wurde der arithmetische Mittelwert gebildet, sodass die Grafiken auf einer vereinfachten Darstellung über eine Durchschnittsberechnung pro Sekunde beruhen. 


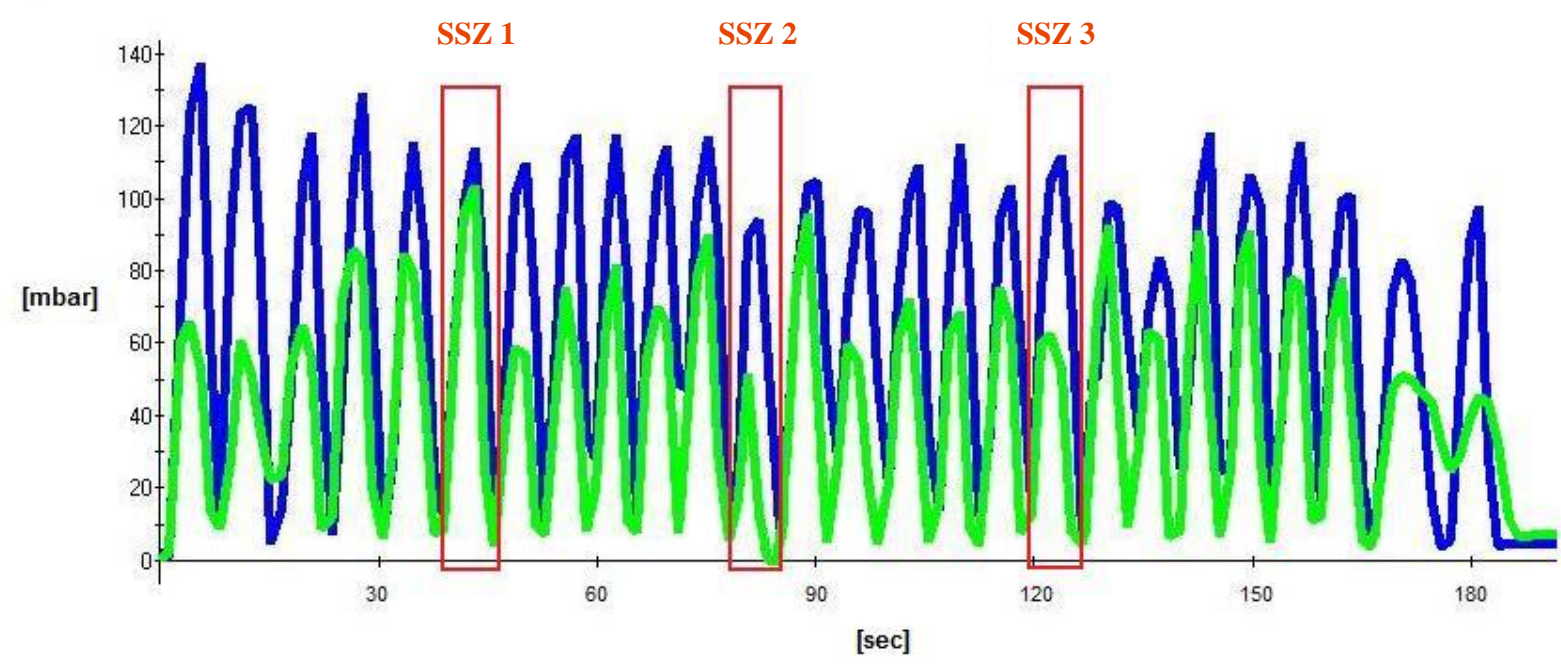

Abbildung 11 Druckdiagramm eines Probanden; negativer Druck wird als nach oben gerichteter Kurvenausschlag im positiven Bereich des Koordinatensystems dargestellt (in mbar)

Da über einen zweikanaligen Sensor des Manometers aufgezeichnet wurde, entstehen in der grafischen Darstellung zwei Kurven: Die blau dargestellte Kurve bezeichnet den Druck im Gaumenkompartiment, der grüne Kurvenverlauf gibt den Druck im Wangenkompartiment wieder. Die Darstellung der Schluckakte erfolgt entsprechend der Arbeiten von Engelke et al. (2010) und Santander et al. (2013) in einem Druck-Zeitdiagramm, in dem die Abszisse den Zeitverlauf und die Ordinate die Druckamplitude in mbar darstellt. Auf der Ordinate wird negativer Druck im positiven Bereich angezeigt.

Aus den Druckamplituden wurden die Schluckvorgänge ermittelt: Jeder Saugschluckzyklus stellt sich als Peak im Kurvenverlauf dar. Die vom Probanden durchgeführten Mundöffnungen entsprechen dem Druckabfall der Kurven zwischen den Saugschluckzyklen. Die 3 Peaks der ausgewählten Zyklen (SSZ 1-3) sowohl der blauen als auch der grünen Kurve wurden mithilfe von GSOFT3050 an ihrem Amplitudenmaximum gemessen und ausgewertet. Bei den erhaltenen Werten handelt es sich demzufolge um die Druckmaxima für jeden der drei Saugschluckzyklen, aufgetrennt nach dem Wangenkompartiment (BFK 1) und dem subpalatinalen Kompartiment (BFK 2).

Maximal mögliche Messfehler wurden mit 5 mbar angenommen. Sie können durch eine eventuelle Wassersäule im Schlauchsystem entstehen, die sich zwischen Messort und Wasserabscheider auf $5 \mathrm{~cm}$ Schlauchlänge entwickelt. 


\subsubsection{Magnetresonanztomographie}

Die Bilddaten wurden auf einer Leonardo Workstation (Siemens Medical Solutions, Forchheim, Deutschland) verarbeitet und im DICOM-Format als CD-Rom exportiert. Anschließende Schritte zur Bildauswertung fanden mithilfe des radiologischen DICOMDarstellungs- und Verarbeitungsprogramms Osirix (OsiriX v.2.6.1b9, Open Source PACS Workstation (Aycan Digitalsysteme, Würzburg, Germany)) statt.

Durch die Versuchsanordnung vorgegeben, konnten die bereits manometrisch eindeutig identifizierten Saugschluckzyklen auch in den Bildsequenzen wiedergefunden werden. Die zur Analyse gewählten drei Zyklen wurden für jeden Probanden nach folgendem Schema ausgewertet.

Pro Saugschluckzyklus wurden 4 repräsentative Bilder zur Auswertung herangezogen, die folgende Aktivitäten darstellen:

Aktivität 1 ist der Lippenschluss zu Beginn eines Saugschluckzyklus

Aktivität 2 ist die Präterminale Saugphase (vorletzter frame des Saugens)

Aktivität 3 ist die Terminale Saugphase (letzter frame des Saugens)

Aktivität 4 zeigt den Velopharyngealen Verschluss beim Schluckvorgang. 
A.

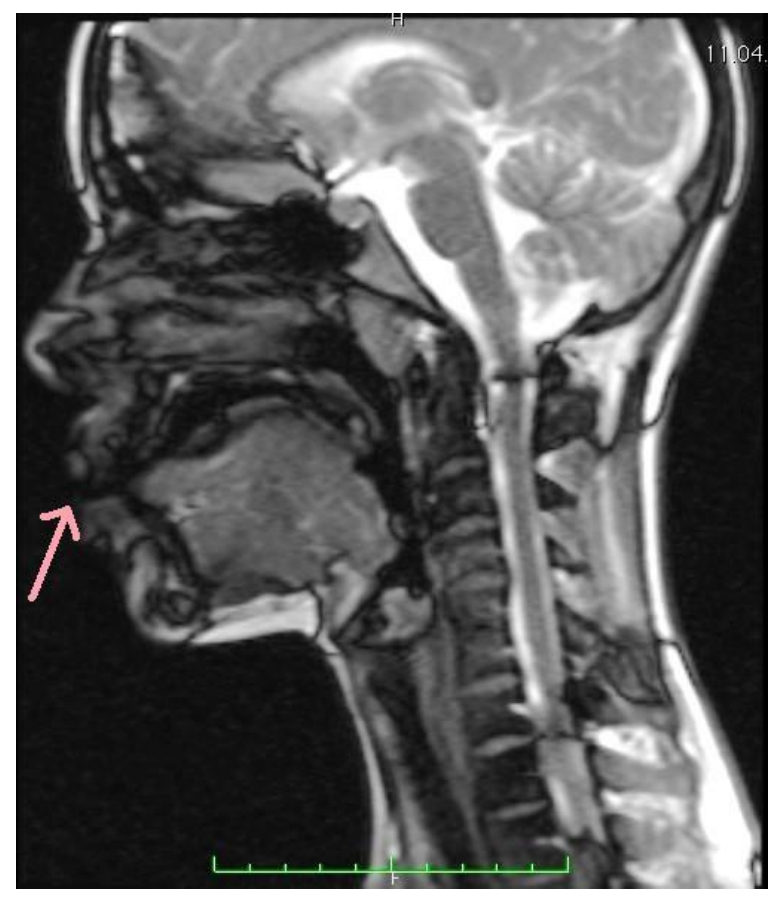

C.

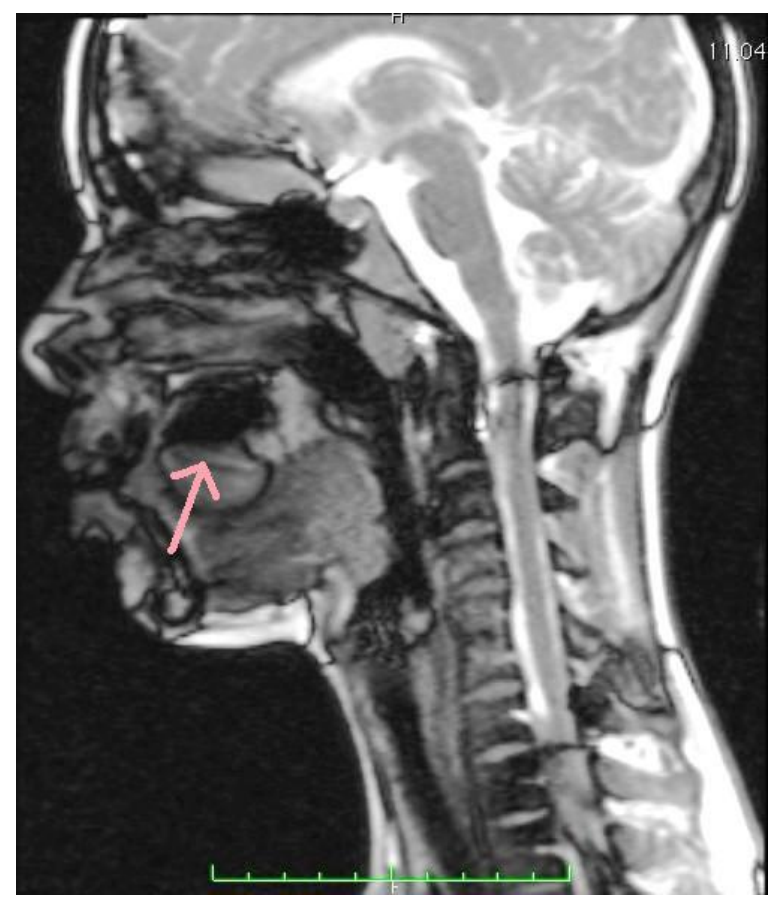

B.

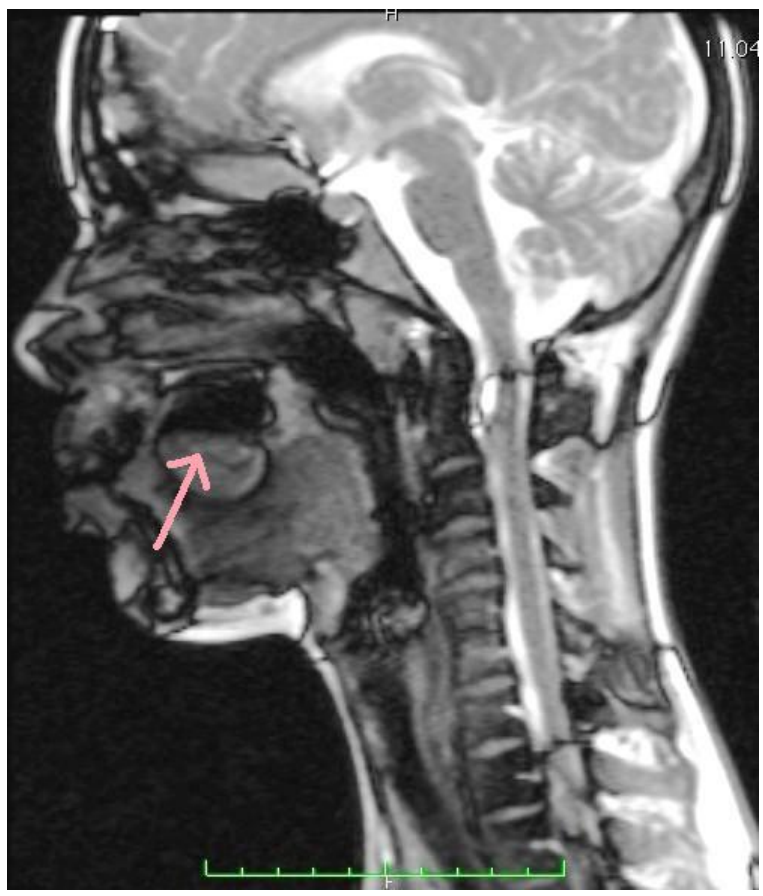

D.

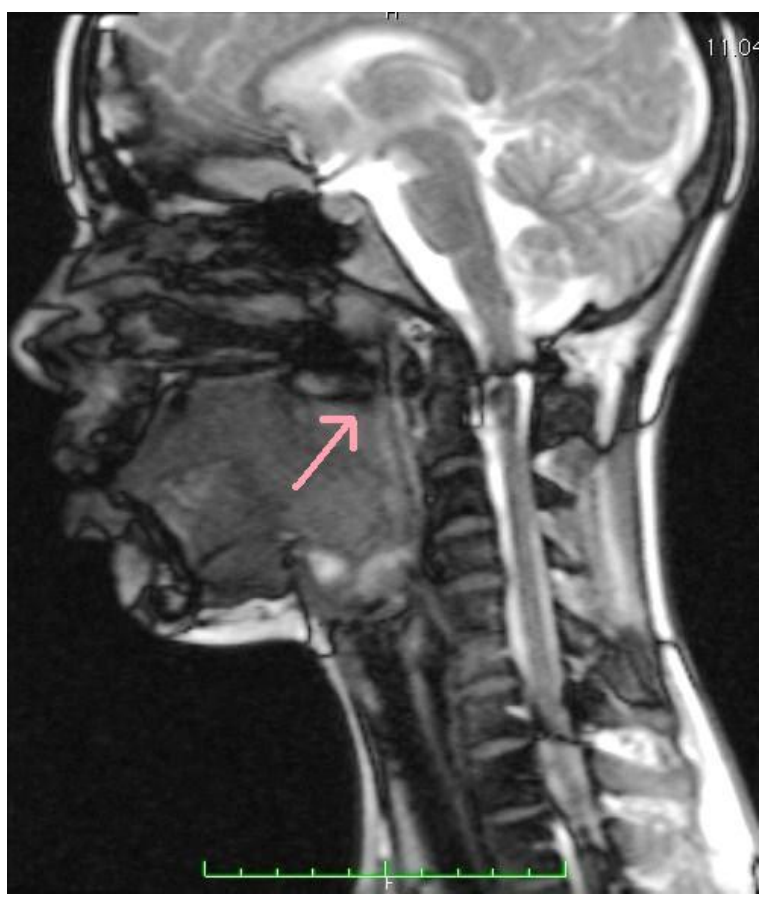

Abbildung 12 Auswahl von 4 repräsentativen Aktivitäts-Bildern eines Saugschluckzyklus: A. Aktivität 1, Pfeil zeigt auf geschlossene Lippen. B. Aktivität 2, Pfeil zeigt in der Präterminalen Saugphase auf die Zunge, über der sich ein dunkel erscheinender Saugraum 
zwischen Zunge und Gaumen darstellt. C. Aktivität 3, Pfeil weist während der Terminalen Saugphase auf die Zunge, die mit Voranschreiten des Saugvorgangs eine Bewegung vollzieht, durch die sie den Saugraum vergrößert. D. Aktivität 4, Pfeil deutet auf den Velopharyngealen Verschluss während der pharyngealer Phase. Kaudal des Velopharyngealen Verschlusses ist eine Verlagerung der dorsalen Zungenoberfläche zu sehen

Zur Auswertung der Bilder wurden anatomische Strukturen innerhalb des mediosagittalen Schnittbildes festgelegt, deren Bewegungsdynamik bei jedem Saugschluckzyklus genauer untersucht werden soll. $\mathrm{Zu}$ deren Identifizierung und für eine objektivierte Nachvollziehbarkeit ihrer Bewegungen wurden Messpunkte und Messflächen, teils innerhalb eines Referenzrechtecks, konstruiert.

Dieses Rechteck wird als Referenzstruktur für eine individuelle planimetrische Analyse der Funktionsräume und -einheiten auf die MRT-Bilder projiziert. Die Grenzlinien dieses Rechtecks sind folgendermaßen definiert:

Die kraniale Begrenzung verläuft tangential entlang des Nasenbodens (NB). Posterior endet das Rechteck an der anterioren Kontur des Dens (D). Kaudal reicht das Rechteck bis auf die Höhe der Oberkante des sechsten Halswirbelkörpers (HWK 6). Nach anterior reicht es bis zum Inzisalpunkt (IP).

Durch das Messrechteck werden die beim Saugschluckzyklus aktiven Strukturen in einen geometrischen Rahmen integriert, innerhalb dessen ihre Verlagerungen stattfinden. Diese werden anhand der entsprechenden Messpunkte verfolgt. Das Messfenster wird individuell definiert und für alle Messungen auf das mediosagittale Schnittbild projiziert. Die Verlagerung der anatomischen Einheiten wird beobachtet, indem geometrisch konstruierte Areale zwischen ihnen und dem Referenzrechteck angelegt werden. Es ergeben sich Flächen, die eine relative Lageveränderung von funktionellen Einheiten anzeigen.

Es werden folgende Messpunkte festgelegt:

SNA: spina nasalis anterior

IM: inferiorster Punkt der Mandibula

IV: inferiorster Punkt des velum palatinum

IMB: inferiorster Punkt der Mundbodenkontur

ES: Epiglottisspitze 
Daraus abgeleitet wurden folgende Messungen durchgeführt:

Unterkieferdistanz: Zur Beschreibung der Unterkieferposition wurde die Distanz zwischen SNA und IM gemessen.

Saugraum: Hypodense Fläche zwischen Gaumen und Zunge, die dem subpalatinalen Kompartiment (BFK 2) entspricht. Sofern eine vordere Begrenzung durch die Zunge oder anderweitig nicht gegeben war, wurden die Lippen als Bezug gewählt.

Unter Zuhilfenahme des Rechtecks werden mit den o.a. Messpunkten folgende Flächen definiert:

Retrolinguale Fläche (RLF) zwischen dorsaler Zungenkontur, posteriorer Rechtecksbegrenzung sowie zwei horizontalen Rechtecksparallelen, ausgehend von IV und ES

Epipharyngeale Fläche (EPF) zwischen kranialer Velumkontur, superiorer und posteriorer Rechtecksbegrenzung sowie der horizontalen Rechtecksparallelen, ausgehend von IV

Externe Mundbodenfläche (EMF) zwischen Submentalkontur, den anterioren und inferioren Rechtecksbegrenzungen sowie einer horizontalen Rechtecksparallelen, ausgehend von IMB 


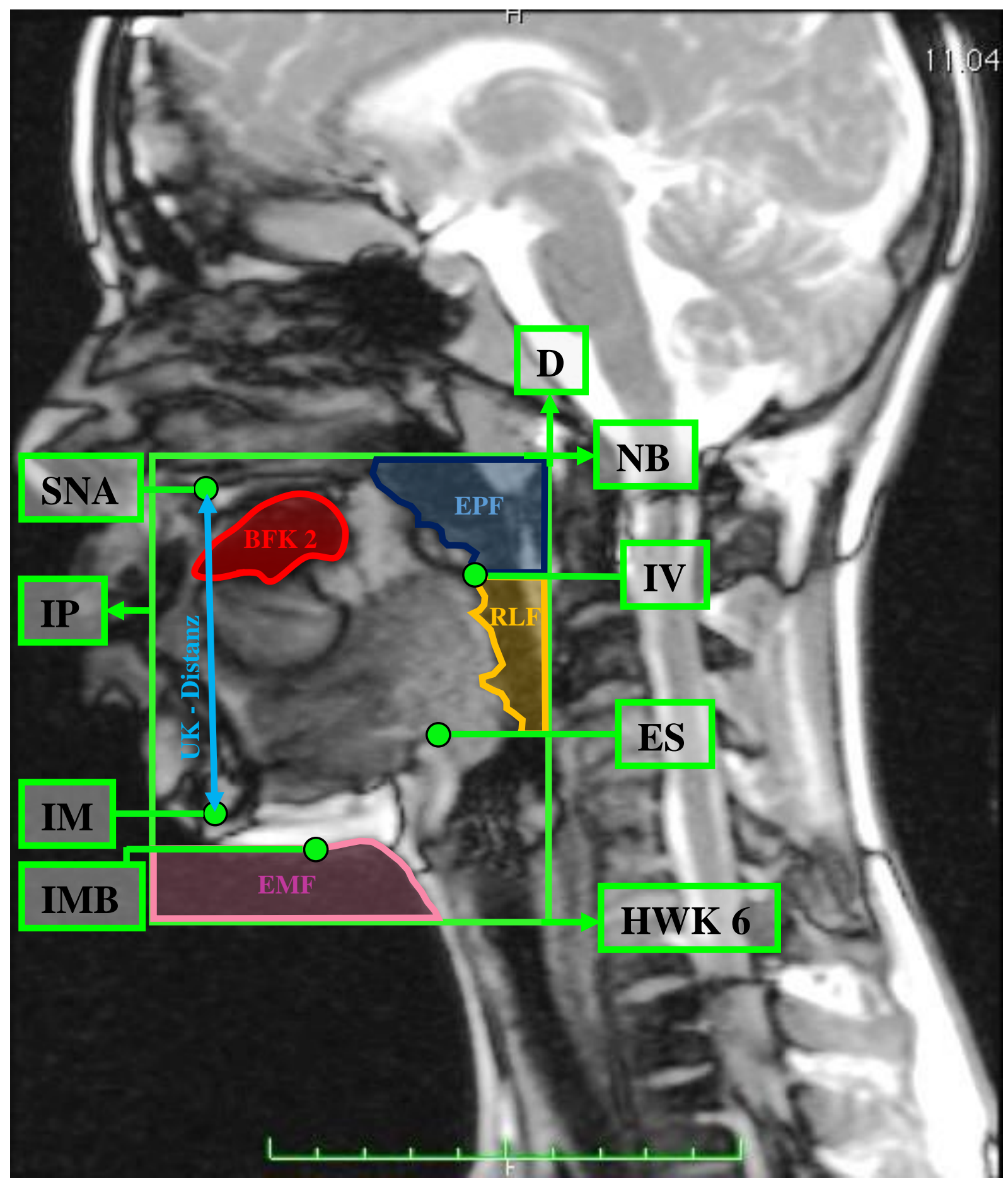

Abbildung 13 MRT-Bild eines Probanden in der mediosagittalen Ebene mit projiziertem Messrechteck, Messpunkten und Messflächen: SNA: spina nasalis anterior; IM: inferiorster Punkt der Mandibula; IV: inferiorster Punkt des velum palatinum; IMB: inferiorster Punkt der Mundbodenkontur; ES: Epiglottisspitze; NB: Nasenbodenlinie; D: anteriore Kontur des Dens; HWK 6: Oberkante des 6. Halswirbelkörpers; IP: Inzisalpunkt; BFK 2: Fläche des Saugraums; EPF: epipharyngeale Fläche; RLF: retrolinguale Fläche, EMF: externe Mundbodenfläche 
Durch die von der jeweiligen Struktur definierten Flächenveränderungen, die sich zwischen den einzelnen Bildern ergeben, werden quantitative Aussagen über Bewegungsrichtung und Bewegungsausmaß möglich. Für die Unterkieferdynamik (Distanz zwischen SNA und IM) und die Fläche des „Saugraumes“ (BFK 2) gingen absolute Werte in die statistische Auswertung ein, da es sich um ,geschlossene“ Strukturen handelt. Die Flächenkonstruktionen für das Velum, die Zunge und die Submentalkontur sind abhängig vom Referenzrechteck, das in seiner Ausdehnung den unterschiedlichen anatomischen Gegebenheiten der Probanden folgt und daher interindividuell verschieden ist. Daher haben die Flächengesamtwerte für das Velum, die Zunge und die Submentalregion isoliert betrachtet keine Aussagekraft. Die Weichteilverlagerungen wurden über relative Flächenänderungen diagnostiziert.

Für jeden untersuchten Parameter wurde von allen vermessenen Bildern derselben Aktivität und derselben Phase der Mittelwert berechnet. Die Konfidenzintervalle (KI) geben den Bereich an, in dem $95 \%$ der Ergebnisse liegen.

\subsection{Statistische Auswertung}

Die erhobenen Daten wurden in Zusammenarbeit mit dem Institut für medizinische Statistik im Zentrum Informatik, Statistik und Epidemiologie der Universitätsmedizin Göttingen ausgewertet. Sämtliche Analysen und Grafiken wurden mit Microsoft Excel 2013 (Microsoft Corporation, Redmond, USA) und den Statistikprogrammen SAS 9.3 (SAS Institute Inc. Cary, USA) sowie Statistica Version 10 (StatSoft GmbH, Hamburg, Deutschland) durchgeführt und erstellt.

Deskriptive Statistiken der manometrischen Daten sowie der MRT-Messwerte beinhalten Mittelwerte und Konfidenzintervalle für die Parameter „Druck“, „Unterkieferdistanz“, „Saugraum“, „Retrolinguale Fläche“, „Epipharyngeale Fläche“ und „Externe Mundbodenfläche“, stratifiziert nach den untersuchten Saugschluckzyklen (SSZ 1-3) und den Aktivitäten „Lippenschluss“, „Präterminale Saugphase“, „Terminale Saugphase“ und „Velopharyngealer Verschluss“. 
Um Unterschiede zwischen den Aktivitäten und den Saugschluckzyklen zu testen, wurde eine Varianzanalyse mit Messwiederholungen (repeated-measures ANOVA) durchgeführt. Für paarweise Vergleiche (sequenzielle Kontraste) wurde ein t-Test für verbundene Stichproben vorgenommen. Das Signifikanzniveau wurde auf $5 \%$ festgelegt. Eine Adjustierung bezüglich multiplen Testens erfolgte mit der Bonferroni-Korrektur.

Zur grafischen Veranschaulichung wurden Abbildungen erstellt, die Mittelwerte und Konfidenzintervalle beinhalten. 


\section{Ergebnisse}

Es wurden 54 Druckmessungen (9 Probanden mit jeweils 3 Saugschluckzyklen an 2 Positionen) und 108 Bilder (9 Probanden mit jeweils 3 Saugschluckzyklen, die aus jeweils 4 Aktivitätsbildern bestehen) ausgewertet.

\subsection{Manometrie}

\begin{tabular}{|c|c|c|c|c|c|c|}
\cline { 2 - 7 } \multicolumn{1}{c|}{} & $\begin{array}{c}\text { Interokkl. } \\
\text { Kompartiment }\end{array}$ & $\begin{array}{c}\text { KI } \\
-95 \%\end{array}$ & $\begin{array}{c}\text { KI } \\
+95 \%\end{array}$ & $\begin{array}{c}\text { Subpalatinales } \\
\text { Kompartiment }\end{array}$ & $\begin{array}{c}\text { KI } \\
-95 \%\end{array}$ & $\begin{array}{c}\text { KI } \\
+95 \%\end{array}$ \\
\hline SSZ 1 & $\mathbf{- 6 6 . 6 2}$ & -88.81 & -44.43 & $\mathbf{- 9 0 . 4 1}$ & -109.79 & -71.03 \\
\hline SSZ 2 & $\mathbf{- 7 4 . 0 3}$ & -92.88 & -55.18 & $\mathbf{- 9 4 . 1 8}$ & -113.55 & -74.81 \\
\hline SSZ 3 & $\mathbf{- 6 2 . 5 9}$ & -92.90 & -32.28 & $\mathbf{- 8 2 . 1 6}$ & -101.41 & 62.90 \\
\hline
\end{tabular}

Tabelle 1 Druckmittelwerte (in mbar) der Amplitudenmaxima aller Probanden aus den 3 ausgewählten Saugschluckzyklen (SSZ 1-3) mit Konfidenzintervallen (KI), aufgeteilt nach den Messorten „Interokklusales Kompartiment“ (BFK 1) und „Subpalatinales Kompartiment"“ (BFK 2)

Die in den individuellen Druckdiagrammen beobachteten Peaks stellen das manometrische Korrelat der Saugschluckzyklen dar. Dabei entspricht die Mundöffnungsphase zwischen den Saugschluckzyklen dem Druckabfall zwischen den Peaks, die sich hierdurch gut voneinander abgrenzen lassen.

Die gemittelten Druckamplitudenmaxima aller Probanden ergaben in jedem Zyklus einen signifikant stärkeren Unterdruck im Subpalatinalen Kompartiment als im Interokklusalen Kompartiment $(\mathrm{p}=0$,0277). Über alle 3 Zyklen gemittelt lag der Durchschnittswert im Interokklusalen Kompartiment bei $-67,75$ mbar, im Subpalatinalen Kompartiment bei -88,92 mbar. Im Vergleich der drei Saugschluckzyklen untereinander ergaben sich keine signifikanten Effekte $(\mathrm{p}=0,0862)$. 


\subsection{Magnetresonanztomographie}

\begin{tabular}{|c|c|c|c|c|c|c|}
\hline & & \multirow{2}{*}{$\begin{array}{c}\text { Distanz } \\
\text { SNA-IM (cm) }\end{array}$} & \multicolumn{4}{|c|}{ Flächen } \\
\hline & & & BFK $2\left(\mathrm{~cm}^{2}\right)$ & RLF $\left(\mathrm{cm}^{2}\right)$ & EPF $\left(\mathrm{cm}^{2}\right)$ & $\operatorname{EMF}\left(\mathrm{cm}^{2}\right)$ \\
\hline \multirow[t]{4}{*}{ SSZ 1} & Lippenschluss & 6.95 & 3.61 & 6.74 & 8.85 & 15.20 \\
\hline & $\begin{array}{c}\text { Präterminale } \\
\text { Saugphase }\end{array}$ & 6.84 & 5.03 & 6.29 & 9.74 & 15.06 \\
\hline & $\begin{array}{l}\text { Terminale } \\
\text { Saugphase }\end{array}$ & 6.88 & 6.05 & 5.99 & 10.31 & 14.79 \\
\hline & $\begin{array}{c}\text { Velopharynx- } \\
\text { Verschluss }\end{array}$ & 6.82 & - & - & 2.63 & 16.32 \\
\hline \multirow[t]{4}{*}{ SSZ 2} & Lippenschluss & 7.01 & 3.28 & 7.15 & 8.90 & 15.39 \\
\hline & $\begin{array}{c}\text { Präterminale } \\
\text { Saugphase }\end{array}$ & 6.87 & 5.15 & 6.70 & 10.00 & 15.35 \\
\hline & $\begin{array}{l}\text { Terminale } \\
\text { Saugphase }\end{array}$ & 6.87 & 5.84 & 6.41 & 10.34 & 15.11 \\
\hline & $\begin{array}{l}\text { Velopharynx- } \\
\text { Verschluss }\end{array}$ & 6.79 & - & - & 3.17 & 16.24 \\
\hline \multirow[t]{4}{*}{ SSZ 3} & Lippenschluss & 7.03 & 5.01 & 7.06 & 9.26 & 15.04 \\
\hline & $\begin{array}{c}\text { Präterminale } \\
\text { Saugphase }\end{array}$ & 6.89 & 4.91 & 7.04 & 10.22 & 15.03 \\
\hline & $\begin{array}{l}\text { Terminale } \\
\text { Saugphase }\end{array}$ & 6.88 & 6.00 & 6.96 & 11.13 & 15.17 \\
\hline & $\begin{array}{c}\text { Velopharynx- } \\
\text { Verschluss }\end{array}$ & 6.81 & - & - & 3.24 & 16.52 \\
\hline
\end{tabular}

Tabelle 2 Mittelwerte der erhobenen Längen- und Flächendaten der vermessenen Parameter 


\subsubsection{Unterkieferdistanz}

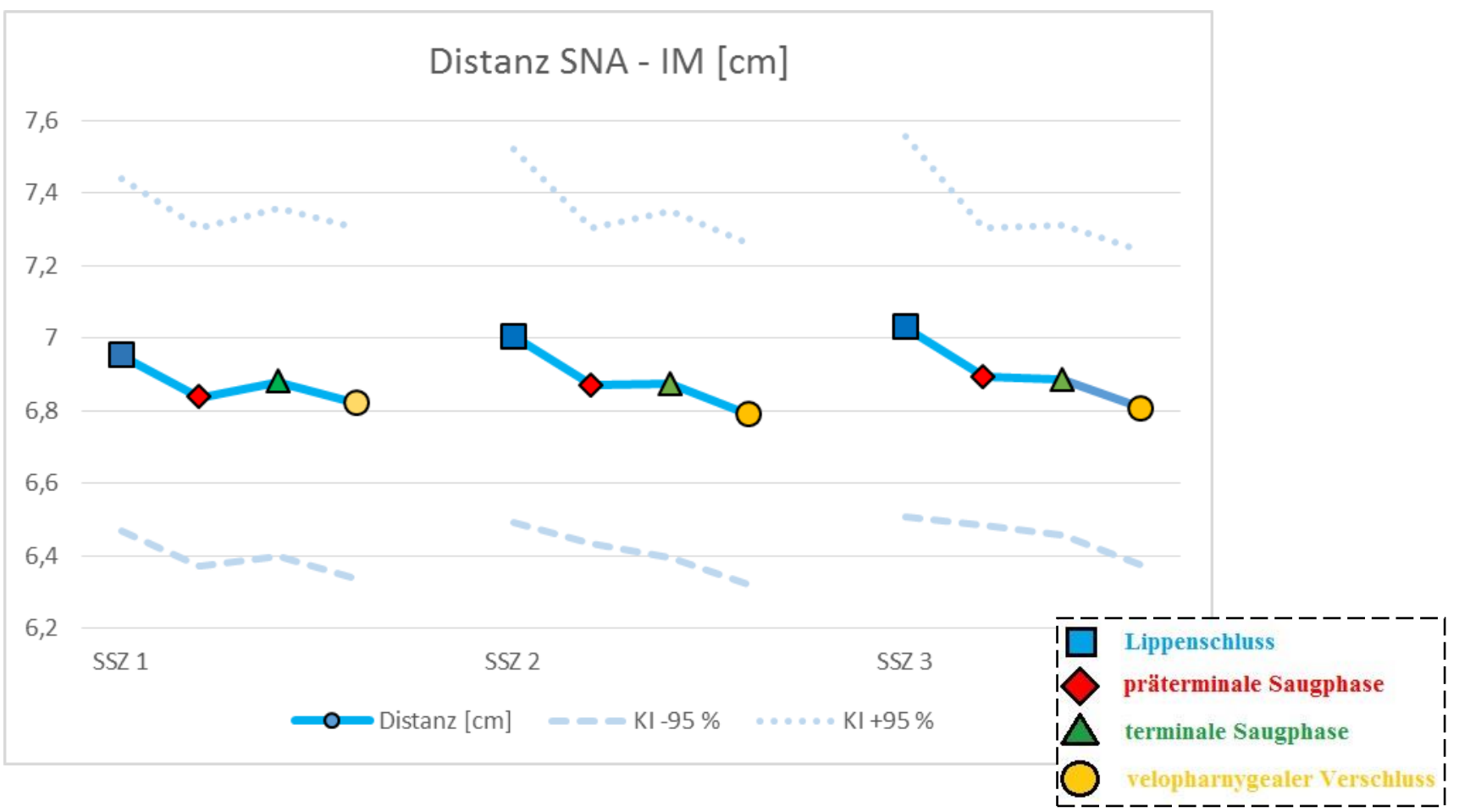

Abbildung 14 Darstellung der Distanz zwischen SNA und IM zur Untersuchung der Unterkieferposition. Angezeigt werden die Mittelwerte und die Konfidenzintervalle (KI). Auf der Ordinate erscheinen die absoluten Messwerte in $\mathrm{cm}$

Gemäß der in Kapitel 2 beschriebenen Methodik wurden die errechneten Mittelwerte der Distanz zwischen SNA und IM sowie ihre Konfidenzintervalle in eine Grafik überführt. Die Ordinate stellt die absoluten Distanzwerte in $\mathrm{cm}$ dar.

Während eines Saugschluckzyklus variiert die Distanz zwischen SNA und IM signifikant ( $\mathrm{p}=$ 0,0004). Die größten Distanzen von etwa $7 \mathrm{~cm}$ treten zu Beginn der Saugschluckzyklen beim Lippenschluss auf. Zur Präterminalen Saugphase hin nimmt die Distanz signifikant ab ( $\mathrm{p}=$ 0,0363). Zwischen Präterminaler und Terminaler Saugphase besteht eine leichte, nicht signifikante Distanzvergrößerung, bevor die Distanz ihre geringsten Werte (MW: 6,81 cm) beim Velopharyngealen Verschluss zeigt $(p=0,0276)$. Zwischen den einzelnen Saugschluckzyklen sind keine signifikanten Effekte feststellbar.

Zwischen der Lippenschlussposition und der Unterkieferposition beim Schlucken mit Velopharyngealem Verschluss liegen 1,9 mm Differenz ( $p=0,0067)$. 


\subsubsection{Saugraum (Subpalatinales Kompartiment / BFK 2)}

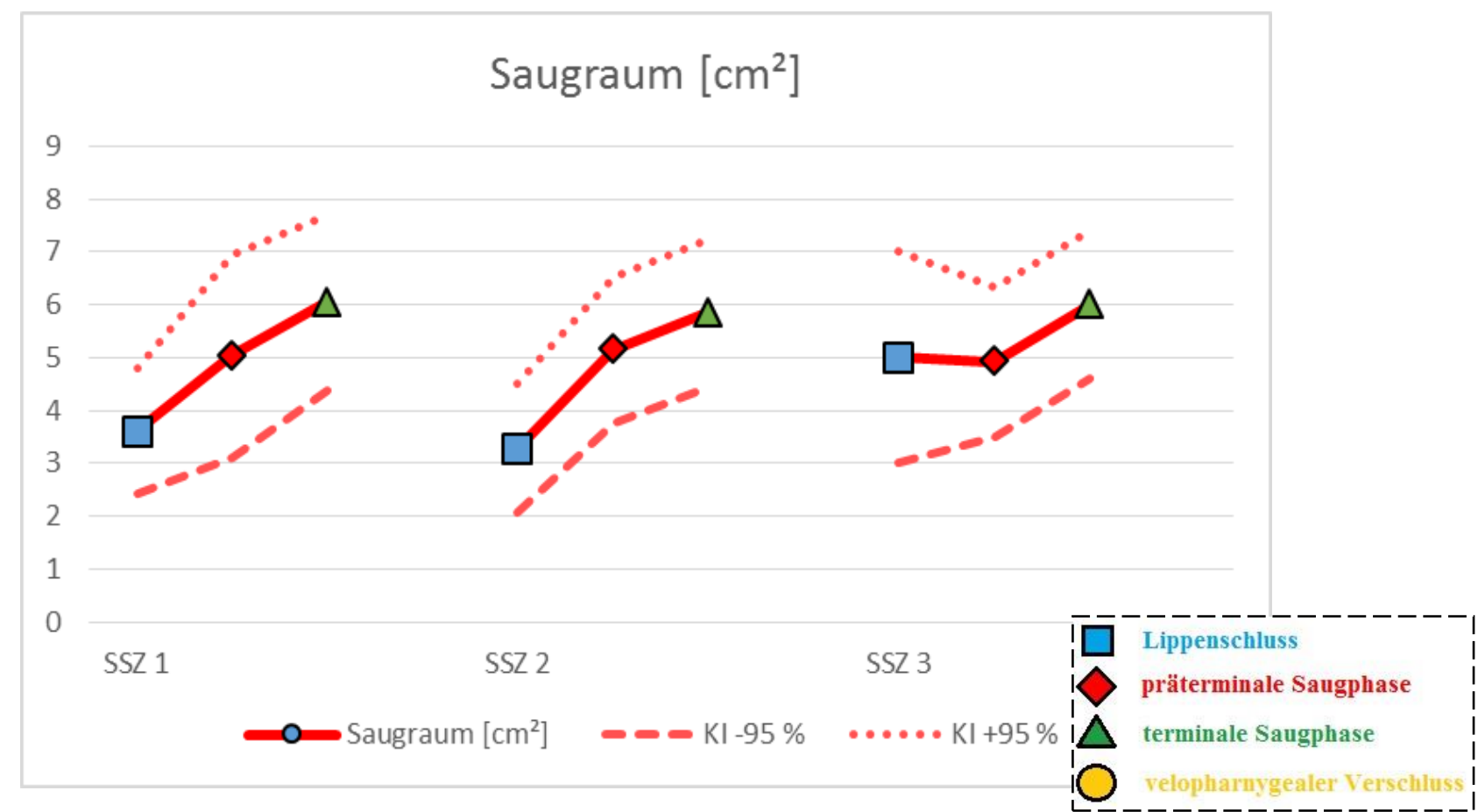

Abbildung 15 Darstellung der mediosagittalen Flächengröße des Saugraums. Angezeigt werden die Mittelwerte und die Konfidenzintervalle (KI). Der Velopharynxverschluss während des Schluckens (Aktivität 4) ist nicht dargestellt, da hierbei aufgrund von Artefakten keine vermessbare Fläche auszumachen war. Auf der Ordinate erscheinen die absoluten Messwerte in $\mathrm{cm}^{2}$

Vom Lippenschluss bis zur Terminalen Saugphase hin findet eine Flächenvergrößerung als Ausdruck einer Expansion des Saugraums statt $(p=0,0156)$. Zwischen Lippenschluss und Präterminaler Saugphase besteht eine nicht signifikante Vergrößerung der Fläche von 1,07 $\mathrm{cm}^{2}(\mathrm{p}=0,179)$. Hochgradig signifikant hingegen ist die Flächenvergrößerung zur Terminalen Saugphase hin $(\mathrm{p}=0,0002)$. Zwischen den einzelnen Saugschluckzyklen sind keine signifikanten Effekte feststellbar. 


\subsubsection{Retrolinguale Fläche}

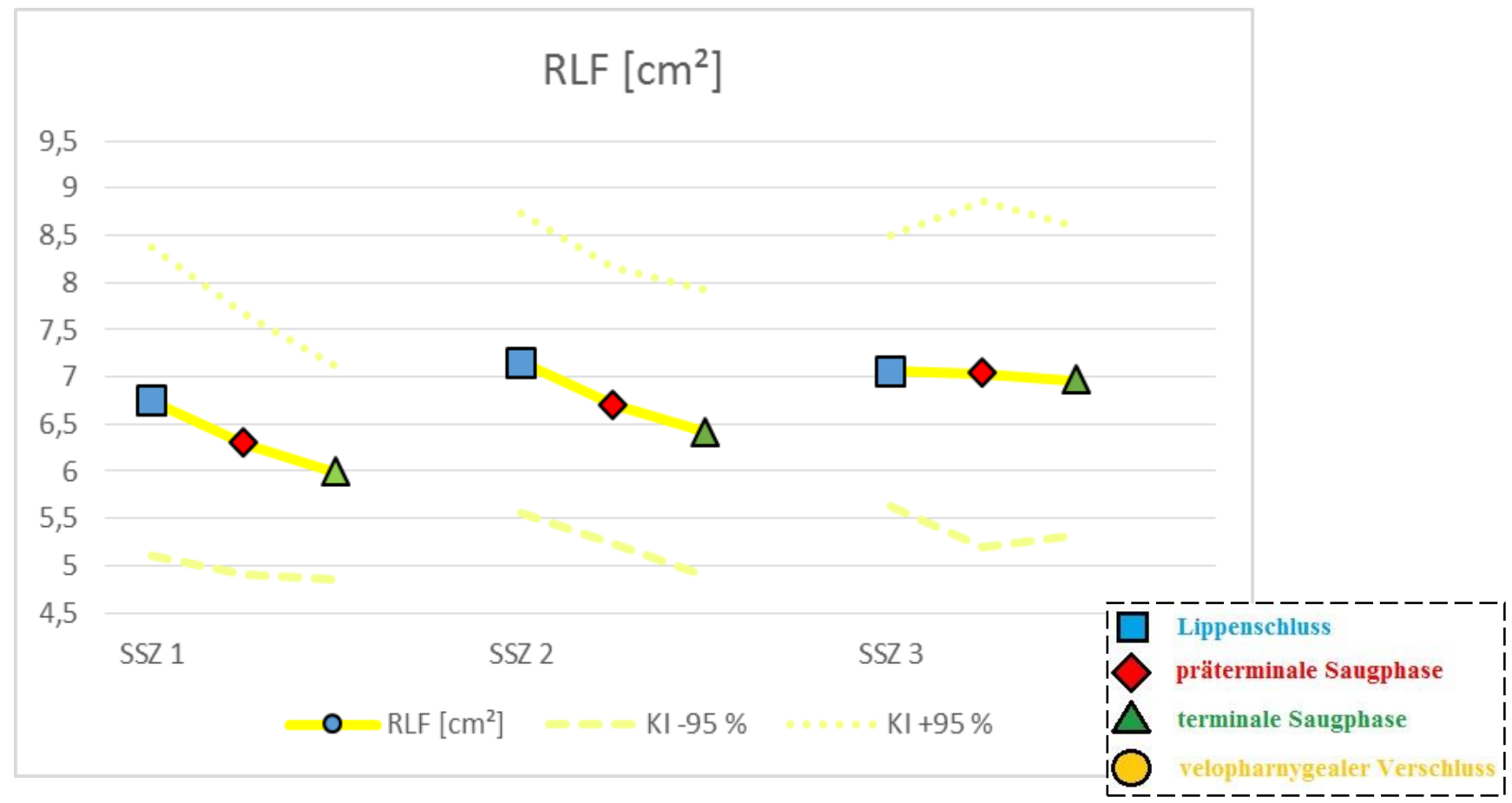

Abbildung 16 Darstellung der mediosagittalen, retrolingualen Flächengröße. Angezeigt werden die Mittelwerte und die Konfidenzintervalle (KI). Der Velopharynxverschluss (Aktivität 4) ist auch hier nicht dargestellt, da während des Schluckens keine eindeutig zu vermessende Fläche auszumachen war

Festzustellen ist eine kontinuierliche, nicht signifikante Verkleinerung der retrolingualen Fläche, ausgehend vom Lippenschluss bis zur Terminalen Saugphase. Während des Velopharynxverschlusses konnte keine retrolinguale Fläche präzise gemessen werden, da die Fläche durch Bewegungsartefakte verzerrt erschien. Zwischen den einzelnen Saugschluckzyklen 1 bis 3 zeigt sich ein signifikanter Anstieg der Flächengröße ( $p=0,0388)$. 


\subsubsection{Epipharyngeale Fläche}

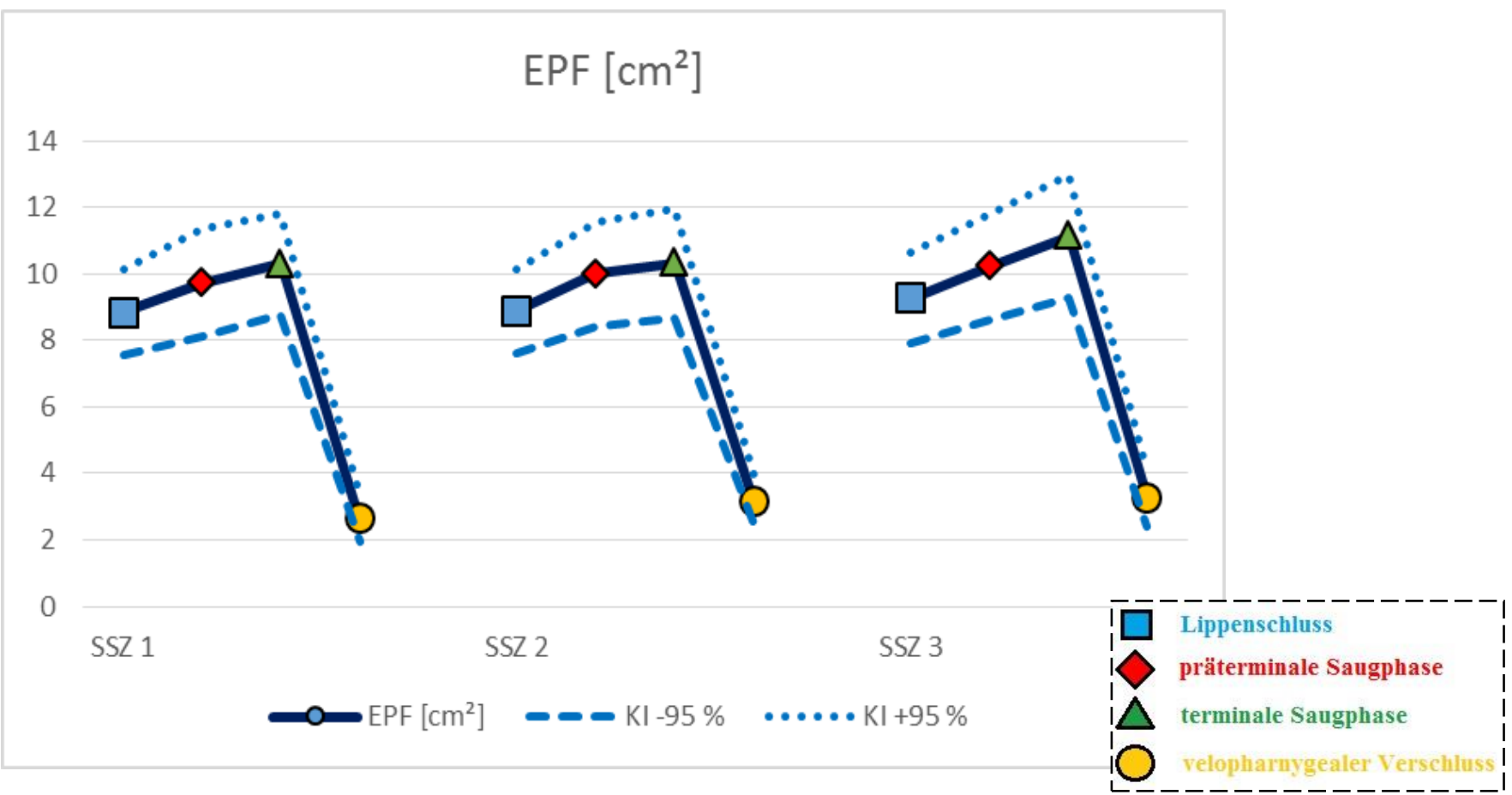

Abbildung 17 Darstellung der mediosagittalen, epipharyngealen Flächengröße zur Beschreibung der velaren Aktivität. Angezeigt werden die Mittelwerte und die Konfidenzintervalle (KI)

Die epipharyngeale Fläche zeigt innerhalb der einzelnen Saugschluckzyklen signifikante Veränderungen zwischen den Aktivitäten $(\mathrm{p}=0,0001)$. Zusätzlich ist ein signifikanter Anstieg zwischen den einzelnen Saugschluckzyklen 1 bis 3 festzustellen $(p=0,0069)$.

Vom Lippenschluss zur Präterminalen Saugphase hin vergrößert sich die Fläche signifikant ( $p$ = 0,0042), während des Saugens findet eine weitere Vergrößerung statt, die jedoch nicht signifikant ist $(\mathrm{p}=0,138)$. Ausgehend von der Terminalen Saugphase reduziert sich die Fläche deutlich und hoch signifikant ( $\mathrm{p}<0,0001)$.

Saugschluckzyklus 1 zeigt im allgemeinen Mittelwert die niedrigsten Werte, gefolgt von Saugschluckzyklus 2 (Unterschied nicht signifikant, p =0,1604). Zwischen Saugschluckzyklen 2 und 3 kommt es zu einem weiteren Anstieg, der signifikant ist $(p=0,0285)$. 


\subsubsection{Externe Mundbodenfläche}

\section{$\operatorname{EMF}\left[\mathrm{cm}^{2}\right]$}

22
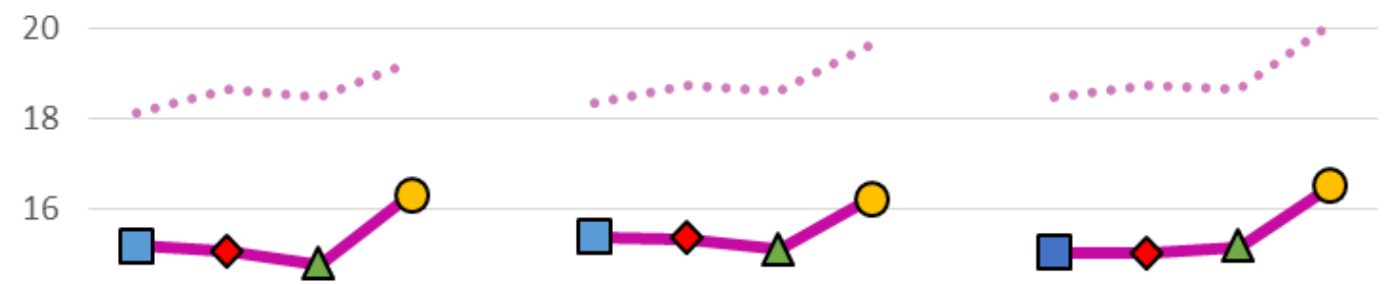

14

12

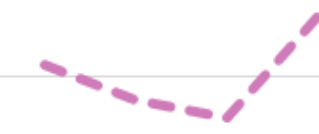

10

SSZ 1

SSZ 2

SSZ 3

$\longrightarrow$ EMF $\left[\mathrm{cm}^{2}\right] \quad--\mathrm{KI}_{-95 \%} \quad \ldots . \mathrm{KI}+95 \%$
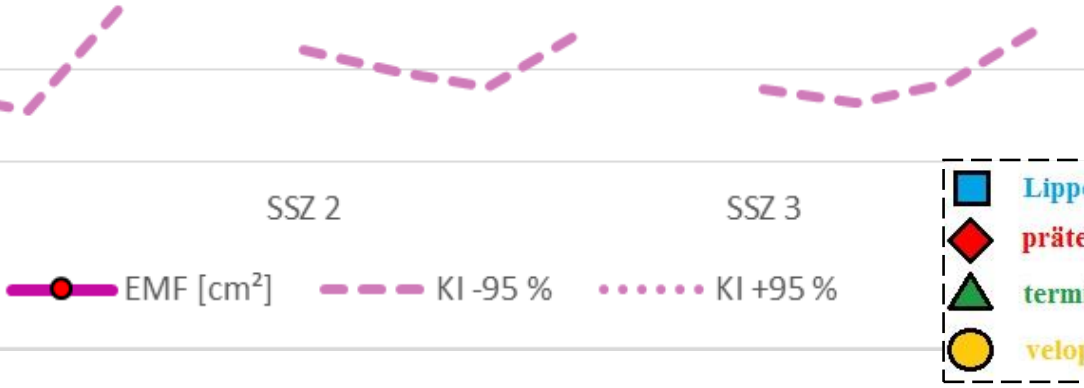

Lippenschluss

präterminale Saugphase

terminale Saugphase

velopharnygealer Verschluss

Abbildung 18 Darstellung der mediosagittalen, externen Mundbodenflächengröße. Angezeigt werden die Mittelwerte und die Konfidenzintervalle (KI)

Vom Lippenschluss bis hin zur Terminalen Saugphase ist eine geringe, nicht signifikante Reduktion der Mundbodenfläche erkennbar $(\mathrm{p}=1)$. Während des Schluckens hebt sich daraufhin der Mundboden, was sich in einer Flächenvergrößerung zwischen Terminaler Saugphase und Velopharynxverschluss niederschlägt. Die Veränderung ist nicht signifikant (p $=0,0714$ ). 


\section{Diskussion}

Eine detaillierte Beschäftigung mit dem Saugvorgang ist von Bedeutung, da er zusammen mit dem Schluckakt zu den grundlegenden, nutritiven Funktionen des orofazialen Organs bei koordinierter Atmungsaktivität zählt (Marik und Kaplan 2003). Die hohe Komplexität von Saugschluckvorgängen macht sie anfällig für Störungen und Beeinträchtigungen, was insbesondere im höheren Alter zutage tritt (Feinberg et al. 1990). Eine adäquate Therapie bedarf einer vorausgehenden, präzisen Diagnostik, vor allem aber eines umfassenden Verständnisses der Vorgänge, die beim physiologischen Saugschluckvorgang ablaufen.

\subsection{Angewendete Methodik}

Bei der Untersuchung des Saugschluckvorgangs ergibt sich die Schwierigkeit, dass die beteiligten Funktionseinheiten einer direkten visuellen Beobachtung nicht zugänglich sind. Der geschlossene Mund sowie die von Weichteilen verdeckten Funktionsabläufe erschweren die Suche nach einer geeigneten Methode zur Diagnostik, die darüber hinaus beachten muss, dass keine Behinderung und dadurch Veränderung der Funktionen mit der angewendeten Methodik einhergeht.

Der Untersuchende trifft neben dem Problem, dass ein Hohlraumsystem den Platz für die Saugschluckfunktion bildet, auf die Herausforderung, die teils nur sehr kurz dauernden, oralen und vor allem pharyngealen Bewegungsvorgänge zum richtigen Zeitpunkt zu erfassen und zu analysieren. Die Wahl der Untersuchungsmethodik hat die jeweilige Fragestellung zu berücksichtigen und erfolgt auf ihrer Grundlage.

Im Allgemeinen gelten ein zu hoher Kostenaufwand, die Zuhilfenahme von Kontrastmitteln sowie Schädigungen durch Röntgenstrahlung als Faktoren, die das Indikationsspektrum vieler der zur Verfügung stehenden Methoden einschränken. Auf dieser Basis rückt die Suche nach den am besten geeigneten Techniken zur Schluckaktuntersuchung in den Fokus (Tamine et al. 2010). 


\subsubsection{Manometrie}

Manometrische Untersuchungen zum Schluckakt bilden einen etablierten und vielfach publizierten Ansatz, der vielen der oben genannten Anforderungen gerecht wird und Informationen physikalischer Art über den Charakter des Hohlraumsystems sowie über Kräfte der Zunge bereitstellt. Alle rein manometrischen Untersuchungen haben den Vorteil, ohne die Einnahme von Kontrastmitteln und ohne eine Schädigung durch Röntgenstrahlen auszukommen (Miller 1982).

Grundsätzlich unterscheidet man Kontaktdrücke, die zwischen Festkörpern wie Geweben gemessen werden (u.a. White et al. 2009), von Drücken, die in Fluiden in einem abgeschlossenen Kompartiment messbar sind (u.a. Engelke et al. 2011). Dieser Unterschied wird in einer Arbeit von Fröhlich et al. (1991) deutlich, in der beide Varianten auftreten.

Die Untersuchung von Kontaktdrücken ist im Hinblick auf eine detaillierte physikalische Saug- und Schluckuntersuchung kritisch zu betrachten: Ein zwischen zwei oder mehreren Körpern gemessener Kontaktdruck vernachlässigt die Analyse umliegender Strukturen. Es resultieren dabei ausschließlich positive Druckwerte, die physikalische Gegebenheiten eventuell nicht vollständig darstellen können. Eine in den Funktionsräumen durchgeführte Manometrie ähnlich Engelke et al. 2011, Santander et al. 2013 oder auch Fränkel (2012) erscheint hingegen sinnvoller und zielführender im Rahmen der Fragestellung nach den allgemein geltenden biomechanischen Verhältnissen beim Saugen und Schlucken. Fränkel untersuchte 2012 mithilfe der Kombination aus Manometrie und pharyngealer Videoendoskopie Schluckphasen bei Tumorpatienten. Die Druckmessungen fanden dabei im subpalatinalen Raum statt. Ähnlich Santander et al. (2013) und Fränkel (2012) erfolgen die Druckmessungen in der vorliegenden Untersuchung auf Grundlage des Biofunktionellen Modells (Engelke 2009), demzufolge Unterdrücke der Schlüsselparameter für die Identifizierung und Beurteilung des Saugvorganges sind.

Im Rahmen unserer Fragestellung dienen die von uns gemessenen Drücke in den Kompartimenten 1 und 2 deshalb der Objektivierung und Identifizierung experimentell aufgestellter, physiologischer Saugschluckzyklen (SSZ) mit intermittierender Mundöffnung. Die manometrisch beobachteten Peaks mit zwischenzeitlichem Druckabfall lassen eine Zuordnung der Aktivitäten „Saugen und Schlucken“ sowie „Mundöffnung“ zu den Druckverläufen zu: Der Saugvorgang schlägt sich im Anstieg des Unterdrucks nieder, dabei sind die Kompartimente voneinander abgegrenzt. Während eine präzise Aufschlüsselung des 
ansteigenden Peak-Anteils zu den Aktivitäten „Saugen und Schlucken“ nicht möglich ist, kann die „Mundöffnung“ zweifelsfrei den zwischenzeitlichen Druckabfällen bis in die Nähe des Umgebungsdrucks zugeordnet werden (vgl. Engelke et al. 2011). Damit können die Saugschluckzyklen voneinander abgegrenzt werden.

In Anlehnung an die Arbeit von Nilsson et al. (1996) wird in der aktuellen Versuchsanordnung das Ansaugen von Flüssigkeit als Stimulus zur Auslösung von Schluckvorgängen eingesetzt. Dabei findet mit einem modifizierten Mundstück einerseits die Boluszuführung, andererseits aber auch die Druckmessung statt, was im Grundschema dem Applikation- und Messsystem der Arbeit von Santander et al. (2013) entspricht. Allerdings wird in unserer Arbeit die Druckmessung zusätzlich im interokklusalen Kompartiment vorgenommen, wodurch das Spektrum erweitert und ein Druckvergleich zwischen zwei verschiedenen Funktionsräumen ermöglicht wird.

\subsubsection{Bildgebende Verfahren}

Eine Visualisierung des Saugens und Schluckens gelingt durch verschiedene diagnostische Methoden, bei denen eine unterschiedliche Beurteilbarkeit hinsichtlich verschiedener Schluckphasen und beteiligter Strukturen besteht.

Als konventionelle Techniken, die den Schwerpunkt auf die Visualisierung der Boluspassage durch den oropharyngealen Trakt legen, gelten die Videofluoroskopie und die Videoendoskopie. Die Videofluoroskopie mit Boluskontrast stellt ein beliebtes Verfahren dar, das den Schluckakt mithilfe eines Barium-angereicherten Bolus wiedergibt. Vorteilhaft im Vergleich zu anderen Methoden ist die Möglichkeit einer kontinuierlichen und nicht unterbrochenen Beobachtung der Vorgänge, insbesondere auch der oralen und pharyngealen Phase. Ihren Status als Standardmethode hat laut Rugiu (2007) die Videofluoroskopie jedoch verloren. Dies hängt auch damit zusammen, dass biologisch schädigende Röntgenstrahlen Anwendung finden. In Anbetracht der Auswahl unserer untersuchten Parameter erscheint das videofluoroskopische Verfahren eher als ungeeignet, um die Verlagerung der Weichteile zu beobachten. Vorteil einer videofluoroskopischen Untersuchung ist eine kontinuierliche Verfolgung des Bolus. Im Rahmen der Zielsetzung der vorliegenden Arbeit ist dies allerdings nicht erforderlich. Das häufig als Kontrastmittel angewendete Barium kann im Aspirationsfall zu unerwünschten Nebenwirkungen führen. Ähnliches gilt für das Verfahren der Videokinematographie, das u.a. von Pokieser 2010 beschrieben wurde. 
Die Einführung der Videoendoskopie, die von Langmore et al. bereits 1988 oder auch Hiss und Postma (2003) beschrieben wurde, arbeitet mit einem transnasal applizierten, flexiblen Endoskop, das die Bildverfolgung auf einem Monitor ermöglicht und dabei ohne Röntgenstrahlung auskommt. Auch eine starre transorale Technik kann zum Einsatz kommen. Die Kosten halten sich niedrig. Vorteilhaft in der Bewertung ist auch, dass Endoskope direkt am bettlägerigen Patienten verfügbar und nicht ortsgebunden sind. Fränkel (2012) hebt die Vorteile der Endoskopie insbesondere bei Tumorpatienten hervor. Nachteilig erweist sich allerdings die Beobachtung der oralen und der ösophagealen Phase. Wegen Verdeckungen und Überlagerungen können einige Strukturen oft nur ungenügend betrachtet werden (Logemann et al. 1998, Böhme 2003). Dazu zählt beispielsweise auch der Mundboden, der allerdings in der vorliegenden Untersuchung in die Beobachtungen mit einbezogen wurde. Während der pharyngealen Phase, die den eigentlichen Schluckvorgang darstellt, kommt es zu White Outs. Dies sind Lichtrückblendungen durch die kontrahierten Muskeln, die die Beurteilung erschweren. Die transnasale Applikationstechnik kann zudem Beschwerden durch Vagusreizungen auslösen (Böhme 2003, Nacci et al. 2008). Die Möglichkeiten einer umfassenderen Abbildung orofazialer Gewebsverlagerungen sind per Endoskopie nicht gegeben. In der endoskopischen Untersuchung des Schluckakts liegt der Fokus auf der Region des Pharynx, wobei die Mundhöhle kaum dargestellt wird. Eine vergleichende Analyse zwischen verschiedenen Untersuchungsteilnehmern ist aufgrund fehlender Standardisierungsmöglichkeiten nur schwer vorstellbar. Die in dieser Arbeit über Flächenveränderungen quantifizierten Gewebsverlagerungen wären mit der Endoskopie demzufolge nicht zu realisieren. Die Fragestellung dieser Arbeit mit Konzentration auf orale Weichgewebe lässt sich auf endoskopische Weise mit hoher Wahrscheinlichkeit nicht beantworten.

Wein et al. (1988, 1993) nutzten die weniger verbreitete Sonographie zur Bildgebung, auch in Kombination mit der Manometrie, und beobachteten die Zungenkinetik; ähnliche Studien fanden durch Neuschäfer-Rube et al. (1997) und Peng et al. (2004) statt. Von während des Ultraschalls auftretenden Artefakten berichtete Grau (2009). Chi-Fishman (2005) sieht ebenfalls in der Sonographie ein nur beschränkt geeignetes Verfahren im Bereich der Schluckdiagnostik. Es kommt zu Schallschatten, die vor allem profund liegende Bereiche schlecht darstellen lassen. Akin et al. (2006) erwähnen eine schwierige Darstellung der oralen und der pharyngealen Phase. Für unsere Untersuchungsziele war es zudem wichtig, eine Vergleichbarkeit zwischen den verschiedenen Probanden herstellen zu können. Die Ausmessung und die Quantifizierung von Flächen, die für die Bewegungsanalyse zuhilfe 
genommen wurden, beruht auf einer standardisierten Positionierung der Probanden im apparativen Untersuchungssystem. Sonographisch sind keine Schnittebenen definierbar, die Applikation ist schwierig reproduzierbar, stark behandlerabhängig und dazu noch intraindividuell verschieden. Insofern erscheint die sonographische Untersuchungsmethodik als kontraindiziert für die Beantwortung der formulierten Fragen.

Die Literatur zeigt, dass die Magnetresonanztomographie als diagnostisches Verfahren auch in der Schluckuntersuchung zunehmend Befürworter und damit Anwendung findet (Anagnostara et al. 2001, Hartl et al. 2003, Breyer et al. 2009; Panebianco et al. 2010, Zhang et al. 2012). Allerdings wird auch deutlich, dass in einigen Aspekten der magnetresonanztomographischen Technik kontroverse Einschätzungen bestehen.

Insbesondere muss dabei die zeitliche Auflösung der Bildaufnahme diskutiert werden. Bereits in den Anfängen der Einführung der Magnetresonanztomographie in die Schluckdiagnostik (Suto et al. 1995) wurde die Technik für detaillierte Bilder der Mundhöhle und des Pharynx bei der Wahl einer zu niedrigen zeitlichen Auflösung als suboptimal eingestuft. Es wurde deutlich, dass eine kontinuierliche Darstellung des Schluckvorgangs mit dem Ziel einer präzisen Analyse, vor allem der kurzen pharyngealen Phase, nur auf Grundlage eines hochauflösenden MRT möglich ist (Anagnostara et al. 2001, Hartl et al. 2003, Panebianco et al. 2010). Mit einer Frequenz von 5 Bildern / s (fps) untersuchten Foucart et al. (1998) 5 gesunde erwachsene Patienten während des Trinkens. Sie bewerten dabei das MRT allerdings als gute Methode, oropharyngeale Aktivitäten nicht nur während des Schluckens, sondern auch zum Beispiel bei der Atmung oder Phonation zu analysieren. Anagnostara et al. (2001) verglichen auf dieser Basis in einer Schluckuntersuchung drei verschiedene, sogenannte high-speed MRI-Systeme miteinander an sieben gesunden Probanden. Auch bei ihnen ist von einer durch Artefakte beeinträchtigten Bildqualität beim Schlucken die Rede. Sofern allerdings Nachteile wie Aspirationsprobleme und die hohen Anwendungskosten gelöst seien, sei ein hoch auflösendes MRT zur Schluckbeurteilung vielversprechend. In Anlehnung an diese Ergebnisse untersuchten Hartl et al. (2003) Schluckvorgänge im MRT bei sieben gesunden Probanden mit Stoffen verschiedener Konsistenz. Zeitliche Aussagen konnten jedoch kaum getroffen werden, die Reihenfolge ablaufender Aktivitäten sei nicht zu bewerten. Panebianco et al. untersuchten 201023 gesunde Probanden und nutzten dabei eine Bildfrequenz von 3-4 fps. Das angewendete Kontrastmittel erlaubte die Evaluation der Bolusprogression, zeitliche Details in Bezug auf das Voranschreiten des Bolus entzogen sich allerdings aufgrund der 
ungenügenden zeitlichen Auflösung einer genaueren Berechnung. Zhang et al. (2012) berichten über eine MRT-gestützte Echtzeitbeobachtung des Schluckvorgangs. Mit fast $25 \mathrm{fps}$ an einem 3-Tesla-Tomographen erzielen sie eine Frequenz, die mit der videofluoroskopischen Auflösung vergleichbar ist.

Im Vergleich zur zeitlichen Auflösung in der Untersuchung von Zhang et al. (2012) wiesen unsere Messungen mit nur etwa 2 fps eine vergleichsweise geringe zeitliche Auflösung auf. Dies ist im Hinblick auf die rasch ablaufenden pharyngealen Prozesse ungenügend, gibt jedoch für die Zielsetzung der Arbeit zur Aktivitätsbeschreibung der funktionellen Strukturen eine hinreichende Information über grundlegende Abläufe der oralen Präparationsphase des Saugschluckzyklus. Allgemein reichen für Beobachtungen der Mundhöhle sowie umgebender Strukturen niedrigere zeitliche Auflösungen aus als für die pharyngeale Phase des Schluckvorgangs, für deren Aktivitätsbeschreibung innerhalb des Pharynx schnellere Frequenzen zu wählen sind.

Der von der Arbeitsgruppe um Suto (1995) erwähnte Aspekt, dass im Falle auftretender Probleme während der laufenden Untersuchung ein Eingriff ärztlicher Seite nur eingeschränkt und verzögert möglich sei, muss beachtet und abgewogen werden. Da an unserer Untersuchung jedoch ausschließlich gesunde Probanden teilnahmen, galt das Risiko von Aspirationen als gering und es kam in keinem Fall zu Schluckbehinderungen, die ein Eingreifen notwendig gemacht hätten. Was die Untersuchung dysphagischer Patienten mit erhöhtem Risiko des Verschluckens und der Bolusaspiration betrifft, so ist die Diagnostik in einer geschlossenen MRT-Röhre durchaus diskutabel und strittig, weil Interventionen nur erschwert bzw. mit zeitlicher Verzögerung vorgenommen werden können. Untersuchungen an gesunden Probanden ohne medizinisch-diagnostische Fragestellung können unseres Erachtens jedoch ohne weiteres im Magnetresonanztomographen durchgeführt werden.

Ein verändertes Bewegungsmuster durch die liegende Position (Suto et al. 1995, Foucart et al. 1998) wird in unseren Untersuchungen nicht angenommen, da bei allen Probanden vor der eigentlichen Versuchsdurchführung zusätzlich Probedurchläufe in liegender Position vorgenommen wurden. Klinisch ergeben sich keine Anhaltspunkte dafür, dass der physiologische Saug-Schluckakt der gesunden Probanden durch die horizontale Position gestört würde. Dies entspricht den Ergebnissen von Zhang et al. (2012). Auch Akin et al. 
(2006), die an 28 Patienten mit front-offenem Biss das magnetresonanztomographische Verfahren testeten und bei ihnen kompensatorische Zungenbewegungen der anterioren und mittleren Zunge zum Schutz des Luftweges feststellen konnten, vernachlässigten Beeinflussungen, die durch die liegende Position der Probanden entstanden sein könnten.

Die Bolusanreicherung mit einem Kontrastmittel, wie sie von Suto et al. (1995) oder auch Anagnostara et al. (2001) nahegelegt wird, verbessert in allererster Linie eine visuelle Verfolgung des Bolus von der Mundhöhle in des Ösophagus, was für die Zwecke unserer Untersuchung nicht von Bedeutung ist, denn im Hinblick auf unsere Fragestellung spielt die Nachvollziehbarkeit der Boluspropulsion keine Rolle. Auf ein Kontrastmittel wurde daher verzichtet. Fluide Medien stellen sich bereits per se kontrastreich im MRT dar (Hartl et al. 2003).

Die Auswahl der analysierten Parameter „Unterkieferdistanz“, „Saugraum“, „retrolinguale Fläche“, „epipharyngeale Fläche“ und „externe Mundbodenfläche“ orientiert sich insbesondere an den Untersuchungen von Anagnostara et al. (2001), Hartl et al. (2003) und Panebianco et al. (2010). Zudem wurde bei der Auswahl auf eine möglichst kontinuierliche Auffindbarkeit im Rahmen der technisch vorgegebenen Bedingungen geachtet. In diesem Zusammenhang wurde beispielsweise auf den Larynx-Hyoid-Komplex (Foucart et al. 1998, Hartl et al. 2003 oder Panebianco et al. 2010) verzichtet, da unter den von uns gewählten MRT-Einstellungen eine zweifelsfreie Zuordnung und Strukturdefinition nicht realisiert werden konnte. Mit der Zunge, deren Bewegung durch den „Saugraum“ und die „retrolinguale Fläche“ beschrieben wird, der Mundbodenregion, die mithilfe der „externen Mundbodenfläche" beobachtet wird sowie dem velum palatinum, das durch die „epipharyngeale Fläche“ geprüft wird, sind wichtige der beim Saugschluckzyklus relevanten Strukturen erfasst. Ergänzend wird die Unterkieferdistanz vermessen. Durch das planimetrische Verfahren (s. Material und Methode) werden die den Saug- und den Schluckvorgang ausmachenden Bewegungen erfasst.

In Anlehnung an Zhang et al. (2012) wird ausschließlich die mediosagittale Achse zur Beurteilung der Bilder gewählt. Von Betrachtungen in den anderen körperlichen Ebenen erwarten wir keine grundlegenden zusätzlichen Resultate, die für das Ziel dieser Arbeit ausschlaggebend wären. 
Im Fazit erweist sich die magnetresonanztomographische Diagnostik mit den von uns gewählten technischen Einstellungen im Rahmen unserer Fragestellung als zielführend und gut geeignet, um die Lageveränderungen der Biofunktionellen Einheiten während des Saugschluckvorgangs zu prüfen. Die Radioneutralität für den Probanden stellt im Allgemeinen ein wichtiges Kriterium für die durchgeführte MRT-Untersuchung dar. Reproduzierbare Kopfpositionen, die im Tomographen eingenommen werden, stellen zudem eine gute Vergleichbarkeit zwischen den entstandenen Bildern her und erlauben damit nicht nur eine gute intra-individuelle Verlaufsdiagnostik, sondern auch, Ähnlichkeiten und Unterschiede zwischen verschiedenen Probanden aufzudecken. Ähnlich Anagnostara et al. (2001), Hartl et al. (2003), Breyer et al. (2009), Panebianco et al. (2010) sowie Zhang et al. (2012) kommen wir bezüglich der diagnostischen Tauglichkeit des MRT-Verfahrens zu dem Schluss, dass es ein geeignetes Instrument zur Evaluierung der anatomischen und physiologischen Charakteristik des Schluckvorgangs darstellt.

\subsubsection{Bewertung des bimodalen Verfahrens}

In der Methodik intraoraler Funktionsdiagnostik stellt die Manometrie einen wichtigen Bereich dar. Da durch sie allein jedoch keine Aussagen über die funktionellen Bewegungsmuster möglich sind, wurden in der vorliegenden Arbeit komplementäre, bewegungsphysiologische Untersuchungen durchgeführt. Sie erlauben es, die Weichteile während des Saugens bei suffizientem Kompartimentschluss zu beobachten. Bezüge visuellphysikalischer Art werden daher ermöglicht und der Anteil einzelner Biofunktioneller Einheiten an der Entstehung des Saugraums, also der Expansion des subpalatinalen Kompartiments, geprüft.

Allgemein bietet eine aus unterschiedlichen Methoden zusammengesetzte Diagnostik des Schluckvorgangs die Chance, umfassendere und erweiterte Untersuchungen durchzuführen. Das gewählte bimodale Untersuchungsverfahren aus Manometrie und MRT ist imstande, die Dynamik zuvor festgelegter anatomischer Parameter unter gleichzeitiger Kontrolle der Kompartimentdrücke zu prüfen. Eine Zuordnung zwischen den beiden Verfahren erfolgte auf Basis einer manometrischen Identifizierung der einzelnen Saugschluckzyklen, die daraufhin den in der Bildgebung erkennbaren und abgrenzbaren Saugschluckzyklen zugeordnet wurden. Dabei stimmte bei jedem Probanden die Anzahl der manometrisch erkennbaren Peaks im Druckdiagramm mit der Anzahl der Saugschluckzyklen im MRT überein. Für weitere 
Untersuchungen wird eine systemische Synchronisierung des Messsystems vorgeschlagen, die aufgrund fehlender technischer Voraussetzungen des in der klinischen Diagnostik eingesetzten MRT-Systems nicht möglich war.

Ähnlich den Arbeiten von Engelke et al. (2010) und Santander et al. (2013) wurde ein Mundstück verwendet, das im Sinne der Fragestellung dieser Untersuchung verändert wurde und zu keinerlei Beeinträchtigung der Saugschluckzyklen führte. Über dieses Mundstück erfolgten die Manometrie und gleichzeitig die Boluszuführung im subpalatinalen Kompartiment. Eine Aspiration des Bolus trat nicht auf. Das Ansaugen einer Flüssigkeit zur Ausübung des Schluckens orientiert sich an der Versuchsdurchführung von Santander et al. 2013. In methodischer Anlehnung an den ROSS-Test (Nilsson et al. 1996) wird wie bei Santander et al. (2013) eine Modifikation des Reservoirbehälters vorgenommen, indem der Bolus aus einer Spritze und nicht aus einem Becher angesaugt wird.

Wasser als Bolus stellt sich meist hell dar und war insbesondere während der Saugphasen zu erkennen. Das Wasser, das im subpalatinalen Kompartiment aus der Schlauchschlinge vom Probanden herausgesaugt wurde, war häufig, aber nicht in allen Bildern im Saugraum zu finden. Die von Hartl et al. (2003) diskutierte Boluswahl kann damit um die Aussage ergänzt werden, dass sich Wasser zwar als Medium aufgrund der Darstellbarkeit im MRT anbietet, sich jedoch nicht in jedem Funktionszustand und in jeder Schluckphase problemlos auffinden lässt. Wichtig ist dabei, den Unterschied zu beachten, der durch die Menge des zugeführten Bolus entsteht. Es wird vermutet, dass auch die geringe Bolusmenge in unserer Studie ausschlaggebend dafür war, dass er in der Bildgebung nicht immer aufzufinden war. Eine verhinderte Darstellung des Bolus kann darüber hinaus auch mit einer Boluslateralisation im Sinne eines Verlassens der mediosagittalen Bildschnittebene zusammenhängen. Für Fragestellungen mit Fokussierung auf den Bolus sollte daher entweder eine größere Bolusmenge genutzt, der Bolus angereichert bzw. ersetzt oder alternative Techniken wie die Videofluoroskopie bevorzugt werden. 


\subsection{Interpretation der Ergebnisse}

\subsubsection{Manometrie}

Im Durchschnitt lag der Höchstwert der Druckamplituden aus den drei ausgewählten Saugschluckzyklen im Kompartiment 1 bei -67,75 mbar, im Kompartiment 2 bei -88,92 mbar. Der Unterschied zwischen diesen Werten ist signifikant.

Von wesentlicher Bedeutung ist die Tatsache, dass die Druckmessungen innerhalb der Kompartimente in Fluiden stattfanden und nicht in einem Kontakt- bzw. Verschlussbereich zwischen verschiedenen Strukturen. Unter der Berücksichtigung von Messfehlern stellten wir keine positiven Drücke während der gesamten Untersuchung fest. Eine Manometrie im subpalatinalen Raum erbrachte bei Santander et al. 2013 bei aktiver Bolusgabe eine mittlere Druckamplitude von etwa -290 mbar. Etwas niedrigere Werte maßen Nilsson et al. 1996 im ROSS-Test. Beide Versuche wurden an erwachsenen Probanden durchgeführt. An Säuglingen wurden in einer früheren Arbeit deutlich niedrigere Druckwerte bis zu -65 mbar festgestellt (Wein et al. 1993). Im Vergleich zu früheren Messungen der Arbeitsgruppe Engelke et al. (2010) wurden in der vorliegenden Untersuchung im Kompartiment 2 mit -88,92 mbar im Vergleich zu -81,86 mbar und im Kompartiment 1 mit -67,75 mbar im Vergleich zu -48,79 mbar ähnliche Werte gemessen. Damit können wir uns der Ansicht anschließen, dass im Gaumenkompartiment im Allgemeinen stärkere Unterdrücke vorzufinden sind als im Wangenkompartiment. Von einer unabhängigen Ausbildung der beiden Funktionsräume ist dabei auszugehen. Der stärkere Unterdruck im Kompartiment 2 könnte auf einer verstärkten Fähigkeit zur Unterdruckbildung beruhen, die von den Wandstrukturen des Saugraumes ausgeht. Im Vergleich dazu sind demzufolge die Muskelaktivitäten rund um den interokklusalen Raum schwächer. Hinzu kommt, dass das subpalatinale Kompartiment während der Funktion des Saugens eine funktionell wichtigere Bedeutung hat als das interokklusale Kompartiment. 


\subsubsection{Unterkieferdistanz}

Zu Beginn jedes Saugschluckzyklus war die Mundöffnungsdistanz am größten. Dies bedeutet, dass der Mund der Probanden in der Aktivität 1 des Lippenschlusses am weitesten geöffnet gewesen ist. Mit Ausnahme eines geschlossenen biofunktionellen Ventils 1 bestehen in dieser Aktivität offene Verschlusssysteme. Die Distanz zwischen spina nasalis anterior und dem mandibulären Messpunkt IM liegt hier durchschnittlich bei 7,00 cm, einzeln betrachtet hat dieser Wert jedoch keine Aussagekraft. Mit zunehmendem Mundschluss verringert sich nun die Distanz. Während der Saugaktivitäten 2 und 3 kommt es dann zu einer Distanzvergrößerung, was als Kaudalbewegung des Unterkiefers zu interpretieren ist. Beim Schluckakt selber (Velopharynxverschluss, Aktivität 4) maßen wir die geringste Mundöffnungsdistanz (MW: 6,81 cm). Der Unterkiefer ist beim Schlucken offenbar in einer oberkiefernahen Position. Es kann bestätigt werden, dass in der pharyngealen Phase des Schluckens ein Zahnreihenschluss zu beobachten ist. In der Differenz zwischen Aktivität 1 und Aktivität 4 ergibt sich ein Wert von 1,9 mm. Die Aktivitäten 1 und 4 entsprechen den Unterkieferpositionen der Ruheschwebelage bzw. der maximalen Interkuspidation. Die Differenz von $1,9 \mathrm{~mm}$ entspricht damit in etwa dem Interokklusalabstand zwischen der Ruheschwebelage in Aktivität 1 und der Schlussbissstellung in Aktivität 4. Der Interokklusalabstand wird mit 2-5 mm angegeben (Reitemeier et al. 2006). Dabei kann die Kopf- und Körperposition die Ruheschwebelage beeinflussen. Die liegende Position der Probanden hält eine Begründung dafür bereit, weshalb der von uns gemessene Wert etwas unterhalb des in der Literatur beschriebenen Abstands liegt: Die Ruheposition des beweglich aufgehängten Unterkiefers wird bei nicht oder kaum kontrahierten Adduktoren in stehender Position maßgeblich von der Schwerkraft beeinflusst. Diese entfaltet jedoch in der liegenden Position kaum mehr eine kaudal wirkende, absinkende Kraft auf den Unterkiefer.

\subsubsection{Saugraum (Subpalatinales Kompartiment / BFK 2)}

Das zwischen Zungenrücken und Gaumen entstehende Biofunktionelle Kompartiment 2 wird in sagittaler Richtung vom vorderen und vom hinteren linguopalatinalen Verschluss begrenzt. Während des Lippenschlusses (Aktivität 1) war die anteriore Begrenzung der Saugraumfläche allerdings zumeist durch die posteriore Lippenkontur gegeben, da die vordere Zunge in dieser Aktivität oft noch keinen anterioren, linguopalatinalen Verschluss (BFV 2) des Saugraumes 
darstellte. In den Saugphasen handelt es sich um die Fläche, die ausschließlich durch die Zungenbewegung formiert und abgegrenzt wird.

Das Kompartiment 2 vergrößert sich kontinuierlich über die Aktivitäten 1, 2 und 3. Die signifikante Erweiterung der Fläche zwischen Präterminaler und Terminaler Saugphase (Aktivitäten 2 und 3) zeigt an, dass das Kompartiment unmittelbar vor dem Schluckakt ein Maximum seiner Expansion erreicht. In unserer Bildfrequenz ergibt sich von Präterminaler zu Terminaler Saugphase, also während einer Dauer von etwa einer halben Sekunde, eine mediosagittale Expansion von etwa $1 \mathrm{~cm}^{2}$. Dass es sich bei dem expandierenden Bereich zwischen Zungenrücken und Gaumen tatsächlich um einen „Saugraum“ handelt, kann durch unser bimethodisches Verfahren bewiesen werden: Der „Saugraum“ entspricht dem subpalatinalen Kompartiment, in dem (neben BFK 1) unsere Druckmessungen vorgenommen wurden. Unmittelbar vor dem Schluckvorgang ist der Bolus hier vorzufinden. Mithilfe des Applikations- und Messsystems wird der Bolus dort appliziert und der subpalatinale Druck gemessen. Gestützt auf die Ergebnisse von Santander et al. (2013) befinden wir uns während der Saugaktivitäten 2 und 3 im aufsteigenden Teil des Druckpeaks. Während der Expansion des Kompartiments 2 nimmt dort demzufolge der negative Druck zu. Die Entstehung eines expandierenden „Saugraums“ ist damit plausibel.

Auch Selley et al. korrelierten bereits 1990 negative Drücke mit der Aufnahme einer Flüssigkeit durch Saugen. Bezogen auf die Beobachtungen des Saugraums sind die vorliegenden Ergebnisse in Einklang zu bringen mit den Arbeiten von Dodds et al. (1990) und Anagnostara et al. (2001). In ihnen wird während der oralen Schluckphase ebenfalls ein subpalatinaler Raum beschrieben, der von einer Löffel-ähnlichen Zungenformation zum harten Gaumen hin gebildet wird.

Während des eigentlichen Schluckens in Aktivität 4 traten Artefakte auf, die eine eindeutige Begrenzung und damit Beschreibung der Fläche nicht mehr zuließen. Erwartet werden kann hier jedoch eine vollständige Anlagerung der Zunge an den Gaumen. Die dabei stattfindenden, schnellen Kontraktionsbewegungen konnten im Rahmen unseres Settings nicht mehr nachvollzogen werden. MRT-Sequenzen mit höherer zeitlicher Auflösung könnten hier dazu verhelfen, die Raumbeschreibungen auch in dieser Schluckphase im Detail fortzusetzen (Zhang et al. 2012).

Ein Saugraum von $5,97 \mathrm{~cm}^{2}$ (MW) sagittaler Ausdehnungsfläche in der Terminalen Saugphase wirft die Frage auf, in welche Richtung das orofaziale System die Expansion 
orientiert und den Platz für sie schaffen kann, um dabei den Unterdruck aufzubauen. Mit unserer Beschreibung der zunehmenden Mundöffnungsdistanz beim Saugen liegt die Hypothese nahe, dass zumindest eine Richtungskomponente der Ausdehnung in kaudale Richtung stattfindet. Zu ähnlichen Ergebnissen kamen auch Selley et al. (1990) sowie Thexton et al. 2004 in videofluoroskopischen Untersuchungen, die im Herabsinken des Unterkiefers eine Begründung für Unterdruckaufbau sahen.

\subsubsection{Retrolinguale Fläche}

Die retrolinguale Fläche (RLF) verringert sich kontinuierlich über die Aktivitäten 1 bis 3 hinweg. Die Interpretation dessen bedeutet eine posteriore Verlagerung der dorsalen Zunge. Damit gibt unsere Untersuchung eine weitere Antwort auf die Frage, wie und wohin Platz für die Expansion des Saugraums geschaffen wird. Die Beobachtungen der retrolingualen Fläche sind nicht signifikant. Sie können die Vergrößerung des subpalatinalen Raumes nicht alleine erklären. Mit der posterioren Zungenverlagerung und einer konsekutiven Verkleinerung des Mesopharynx durch eine Annäherung der Zunge an die posteriore Pharynxwand entsteht nichtsdestotrotz weiterer Platz innerhalb der Mundhöhle, wodurch ein weiterer Faktor für die Saugraumexpansion gegeben ist. Die wichtige Rolle der Zunge bei der Formation eines Saugraumes ist mehrfach beschrieben worden (Selley et al. 1990; German et al. 1992; Thexton et al. 2004). Die Verlagerung des dorsalen Zungenteils nach mesopharyngeal findet sich bei Panebianco et al. (2010). Sie beschrieben analog zu unseren Ergebnissen eine retrale Zungenbewegung in der oralen Schluckphase, die sie im MRT feststellten.

Die Aktivität 4 ist wegen der ungenügenden zeitlichen Auflösung nicht darstellbar. Nach den physiologischen Prozessen ist eine Kontraktion der Pharynxwände gegen den Zungenrücken zu erwarten (Logemann 1988). Die Schnelligkeit dieser Bewegungen ließ keine eindeutig begrenzte retrolinguale Fläche auffinden. Bei Fokussierung auf die pharyngeale Schluckphase ist eine zeitlich höherauflösende Magnetresonanztomographie erforderlich.

Der Vergleich der 3 untersuchten Saugschluckzyklen untereinander ergibt einen signifikanten, allgemeinen Anstieg der Flächengrößen. Demzufolge wird die posteriore Zungenbewegung im Laufe der 3 Minuten Untersuchungsdauer offenbar abgeschwächt, die Intensität lässt nach. Dieses Phänomen lässt sich möglicherweise als Ermüdungseffekt erklären und konnte bereits in einer vorausgehenden Arbeit beobachtet werden (Santander et al. 2013). 


\subsubsection{Epipharyngeale Fläche}

Während des Lippenschluss-Saugvorgangs (Aktivitäten 1 bis 3 ) vergrößert sich die epipharyngeale Fläche (EPF). Beim Schlucken (Aktivität 4) verringert sie sich anschließend deutlich. Die Interpretation dieser Kurvenmorphologie bekräftigt die These, dass das Gaumensegel keine aktive Rolle dabei spielt, den Saugraum zu erweitern. Das velum palatinum scheint unseren Untersuchungen nach beim Saugen eine anteriore Bewegung zu machen. Demzufolge ist anzunehmen, dass die primäre Funktion des Gaumensegels beim Saugen diejenige ist, den posterioren, linguopalatinalen Verschluss durch einen velolingualen Verschluss zu forcieren (Biofunktioneller Verschluss 3). Die Aktivität des $m$. tensor veli palatini scheint demnach ausgeprägter $\mathrm{zu}$ sein als die des $m$. levator veli palatini. Die Spannung des Gaumensegels führt zu einer anterokaudalen Velumbewegung, die dazu beiträgt, den Saugraum nach hinten zu verschließen, indem der posteriore Verschluss des Kompartiments 2 verstärkt wird.

Mit den Ergebnissen einer nach ventral orientierten Velumbewegung können wir uns den Resultaten von Engelke et al. (1995b) anschließen. Sie stellten in elektromagnetischen Untersuchungen ebenfalls ein antero-inferiores Bewegungsmuster des Velum fest, um den velolingualen Verschluss während der oralen Aktivitätsphase aufrecht zu erhalten. Iida et al. bestätigen 2003 in elektromyographischen Versuchen die velare Funktion und ihren Anteil an einem Verschlusssystem, das für die Abgrenzung eines Raumes von Bedeutung ist, in dem sich ein Unterdruck aufbaut.

Während des Schluckens tritt eine deutliche und signifikante Verkleinerung der epipharyngealen Fläche im Sinne einer velaren Elevation zutage. Diese Beobachtung diente der Definition der Aktivität 4: Die Velumelevation führt zum Kontakt mit der posterioren Pharynxwand (Velopharyngealer Verschluss). Durch ihn wird der Inhalt der Mundhöhle (Bolus) nach posterior freigegeben, die Atmung kurzzeitig unterbrochen und die pharyngeale Passage in den Ösophagus unterstützt.

Im Vergleich der Saugschluckzyklen untereinander steigen die EPF-Werte an: Mit 7,88 $\mathrm{cm}^{2}$ (MW) im Saugschluckzyklus 1, 8,10 $\mathrm{cm}^{2}$ (MW) im Saugschluckzyklus 2 und 8,46 $\mathrm{cm}^{2}$ (MW) im Saugschluckzyklus 3 ist eine kontinuierliche Flächenvergrößerung festzustellen, wobei diejenige zwischen den Saugschluckzyklen 2 und 3 signifikant ist $(\mathrm{p}=0,0285)$. Dies kann 
eine allgemein schwächer werdende Velumaktivität bedeuten, bei der das Velum im Laufe der kontinuierlich repetitiven Saugschluckzyklen weniger stark beim Schlucken eleviert.

\subsubsection{Externe Mundbodenfläche}

Die externe Mundbodenkontur sinkt ausgehend vom Lippenschluss bis zur Terminalen Saugphase hin ab, was sich in einer Verringerung der konstruierten Fläche niederschlägt. Eine Vergrößerung der Fläche zeigt sich beim Schlucken. Hier nimmt die Submentalregion demnach eine nach kranial orientierte Bewegung vor. Es liegt nahe, die beobachtete Bewegung der Submentalregion mit der kranialen Bewegung des von ihr bedeckten LarynxHyoid-Komplexes zu korrelieren, die oft beschrieben wurde (Suto et al. 1995, Foucart et al. 1998, Anagnostara et al. 2001, Hartl et al. 2003, Panebianco et al. 2010). Bei Anagnostara et al. (2001) ist von einer maximal hohen Hyoidposition während der pharyngealen Phase die Rede. Die Elevation des Larynx-Hyoid-Komplexes beim Schlucken kann insofern auch in unseren MRT-Messungen nachvollzogen werden.

Zusammenfassend kommt es bei unseren Beobachtungen der strukturellen Verlagerung Biofunktioneller Einheiten zu einer signifikanten Vergrößerung des Saugraumes zwischen Zunge und Gaumen während der Saugphasen, wobei der dort gemessene Unterdruck während des Saugens zunimmt und durchschnittliche Werte von -88,92 mbar annimmt. Der Unterdruck ist damit signifikant stärker als derjenige im interokklusalen Kompartiment (-67,75 mbar). An der Erweiterung des Saugraumes hat die Mobilität des Unterkiefers wahrscheinlich nur geringen Anteil, denn der Unterkiefer bewegt sich während des Saugens in eher schwacher Weise nach kaudal. Während der Expansion des Saugraumes macht die Zungenbasis eine leichte, retrale Bewegung und verkleinert damit das mesopharyngeale Lumen, auch diese Bewegung gibt im Allgemeinen Platz für den Saugraum frei, ist allerdings nicht stark ausgeprägt. Das Gaumensegel verstärkt den Ergebnissen zufolge mit einer anterokaudalen Bewegung die posteriore Begrenzung des Saugraumes. Beim Schluckakt eleviert es und bildet den velopharyngealen Sphinkter. Es bestehen Tendenzen zu einem Absinken des Mundbodens während der Saugphase. In der pharyngealen Schluckphase führt er eine Kranialbewegung aus, die vereinbar mit der Elevation des Larynx-Hyoid-Komplexes ist. 


\subsection{Klinischer Ausblick}

In Anbetracht der äußeren Rahmenbedingungen muss berücksichtigt werden, dass MRTdiagnostische Untersuchungen aufwendiger, zeit- und kostenintensiver sind als die meisten anderen verfügbaren Methoden. Entscheidungskriterium bei der Wahl geeigneter Methoden sollte vor allem die jeweilige diagnostische Fragestellung sein. Patienten-abhängige Faktoren wie allgemeiner Gesundheitszustand, Mobilität und Dringlichkeit der Untersuchung spielen ebenfalls eine große Rolle. Die kombinierte Methodik mit eingesetztem Mundstück zur Manometrie bei gleichzeitiger Bolusapplikation verlangte dem Probanden ein größeres Maß an Compliance ab. Das bimodale Verfahren mit intraoral platzierter Messapparatur kann damit nicht pauschal für Schluckuntersuchungen, insbesondere am dysphagischen Patienten, empfohlen werden, da die Gefahr von Aspirationen erhöht ist und ein ärztliches Einschreiten durch den apparativen Aufbau erschwert ist.

Als Grundlage für reine Forschungszwecke bei gesunden Probanden ohne medizinischdiagnostischen Abklärungsbedarf erweist sich unsere Messmethodik allerdings als zielführend und ergebnisreich im Hinblick auf die zum Ziel gesetzte Klärung unserer Fragen. Insbesondere die sehr gute Standardisierung durch fixierte Positionierung der Probanden, aber auch die automatisierte Bildakquisition sind Grundlage für eine präzise Datenerhebung, die eine Vergleichbarkeit ermöglicht, auf deren Basis die statistischen Analysen konsequent durchgeführt werden konnten. In diesem Aspekt erweisen sich die Endoskopie und die Sonographie als nachteilig, da hierbei nur eine behandlerabhängige, individuelle Durchführung der Untersuchung stattfinden kann, die kaum reproduzierbar ist. Videofluoroskopische Untersuchungen können mit dem Ziel einer kontinuierlichen Bolusdarstellung weiterhin empfohlen werden.

Mithilfe der Magnetresonanztomographie können Erkenntnisse zum Saugschluckvorgang zur Beurteilung von Weichteilbewegungen rund um die Mundhöhle erzielt werden. Gerade für die orale Präparationsphase stellt die Magnetresonanztomographie eine geeignete Technik dar, die Verlagerung funktioneller Einheiten zu erfassen. Im Gegensatz zur pharyngealen Schluckphase sind dabei auch niedriger frequente Aufnahmesequenzen zur Untersuchung geeignet. 


\section{Zusammenfassung}

Der Saugschluckvorgang stellt eine wesentliche Funktion des orofazialen Systems dar. Eine detaillierte Analyse der am Saugschluckvorgang beteiligten anatomischen Strukturen ist für das Verständnis von funktionellen Störungen bedeutsam. Die vorliegende Untersuchung hatte das Ziel, mithilfe der Magnetresonanztomographie Saugschluckzyklen (SSZ) unter manometrischer Kontrolle im Hinblick auf die Verlagerung oropharyngealer Strukturen zu analysieren.

Dazu wurden mediosagittale MRT-Sequenzen von neun gesunden Probanden an einem 3Tesla-MRT mit einer zeitlichen Auflösung von 0,53 s in einem Zeitraum von $180 \mathrm{~s}$ aufgenommen. Die Probanden saugten über ein Mundstück Wasser aus einem Reservoir und führten einen anschließenden Schluckakt aus. Zwischen den repetitiven Saugschluckzyklen wurde der Mund geöffnet.

Zur Auswertung wurden 3 Saugschluckzyklen (SSZ) ausgewählt: SSZ 1 nach 40 s, SSZ 2 nach 80 s, SSZ 3 nach 120 s. Pro Saugschluckzyklus wurden jeweils vier repräsentative Aktivitätsbilder (Lippenschluss, Präterminale und Terminale Saugphase, Velopharynxverschluss) in einem planimetrischen Vermessungsverfahren analysiert. Mithilfe anatomisch definierter Untersuchungsfenster innerhalb der mediosagittalen Schnittbilder konnten die Unterkieferposition, die Dynamik des Saugraums sowie Veränderungen der dorsalen Zunge, des Velum und der Mundbodenposition beobachtet werden.

Folgende Resultate wurden ermittelt:

1) Der subpalatinale Bereich stellt in der vorbereitenden Phase des Schluckvorgangs einen expandierenden Saugraum dar, in dem ein durchschnittliches negatives Druckmaximum von $-88,92$ mbar gemessen werden konnte.

2) Der interokklusale Raum enthält ein durchschnittliches negatives Druckmaximum von $-67,75$ mbar. Damit ist der Unterdruck im subpalatinalen Raum im Vergleich zum interokklusalen Raum signifikant stärker $(\mathrm{p}=0,0277)$. 
3) Die Position des Unterkiefers bewegt sich während des Saugens um 0,2 mm nach kaudal (nicht signifikant). Zwischen der Unterkieferposition bei Lippenschluss und derjenigen beim Velopharynxverschluss liegt eine Differenz von 1,9 mm.

4) Die Fläche des subpalatinalen Saugraums nimmt während des Saugvorgangs signifikant um $0,93 \mathrm{~cm}^{2} \mathrm{zu}(\mathrm{p}=0,0002)$.

5) Während der Saugphasen verkleinert sich die retrolingual konstruierte Messfläche um $0,22 \mathrm{~cm}^{2}$ und verengt durch diese Bewegung das mesopharyngeale Lumen (nicht signifikant). Diese Flächenveränderung ist vereinbar mit einer antero-posterioren Verlagerung der dorsalen Zunge während des Saugens.

6) Das velum palatinum zeigt während des Saugens eine anterokaudale Bewegung, die sich aus der epipharyngealen Flächenvergrößerung um $0,61 \mathrm{~cm}^{2}$ während des Saugens ergibt (nicht signifikant). Am Übergang der Terminalen Saugphase zum Velopharynxverschluss findet eine deutliche Verkleinerung der epipharyngealen Fläche statt (Differenz: 7,58 $\mathrm{cm}^{2}$, signifikant mit $\mathrm{p}<0,0001$ ). Diese Flächenveränderung ist vereinbar mit der Velumelevation zur Bildung des Velopharyngealen Verschlusses beim Schlucken.

7) Die externe Mundbodenkontur sinkt beim Saugen ab (Differenz: 0,12 $\mathrm{cm}^{2}$, nicht signifikant) und führt anschließend in der pharyngealen Schluckphase eine Kranialbewegung aus, die mit der Larynx-Hyoid-Elevation vereinbar ist (Differenz $1,34 \mathrm{~cm}^{2}$, nicht signifikant $(\mathrm{p}=0,0714)$ ).

8) Im Vergleich der einzelnen Saugschluckzyklen treten in der retrolingualen $(p=0,0388)$ und in der epipharyngealen Fläche $(\mathrm{p}=0,0069)$ signifikante Vergrößerungen auf, die als Ermüdungserscheinung im Verlauf wiederholter Saugschluckzyklen interpretiert werden können.

Eine kombinierte Bewegung aus der Verlagerung der Zunge in antero-posteriore Richtung bei forciertem velo-lingualem Kontakt einerseits, und einer Kaudalbewegung des Mundbodens andererseits, scheinen die hauptsächlichen Mechanismen während des Saugvorgangs zu sein und eine Expansion des Saugraums zwischen Zunge und Gaumen zu bewirken. 
Teile dieser Arbeit wurden in der Fachzeitschrift „European Archives of Oto-RhinoLaryngology" veröffentlicht.

Engelke W, Glombek J, Psychogios M, Schneider S, Ellenberger D, Santander P.

Displacement of oropharyngeal structures during suction-swallowing cycles.

Eur Arch Otorhinolaryngol 2014 Feb 9. DOI 10.1007/s00405-014-2919-4 [Epub 2014 Feb 9] 


\section{Anhang}

\subsection{Abkürzungsverzeichnis}

\begin{tabular}{ll} 
A & Fläche \\
BFE & Biofunktionelle Einheit \\
BFK & Biofunktionelles Kompartiment \\
BFV & Biofunktioneller Verschluss \\
cm & Zentimeter \\
D & Dens (anteriore Kontur) \\
EMF & Externe Mundbodenfläche \\
EPF & Epipharyngeale Fläche \\
ES & Epiglottisspitze \\
F & Kraft \\
FEES & Fiberoptic Endoscopic Evaluation of Swallowing \\
fps & Frames pro Sekunde \\
HWK 6 & Sechster Halswirbelkörper (Oberkante) \\
IM & Inferiorster Punkt der Mandibula \\
IMB & Inferiorster Punkt der Mundbodenkontur \\
IP & Inzisalpunkt \\
IV & Inferiorster Punkt des velum palatinum \\
kHz & Kilohertz \\
KI & Konfidenzintervall \\
m. & Musculus \\
mbar & Millibar \\
ml & Milliliter \\
mm & Millimeter \\
mmHG & Millimeter Quecksilbersäule \\
MRI & Magnetic Resonance Imaging \\
MRT & Magnetresonanztomographie \\
\hline &
\end{tabular}




$\begin{array}{ll}\text { ms } & \text { Millisekunde } \\ \text { MTP } & \text { Membrantrichterplatte } \\ \text { MW } & \text { Mittelwert } \\ \text { NB } & \text { Nasenboden } \\ \text { NMR } & \text { Nuclear Magnetic Resonance } \\ \text { p } & \text { p-Wert } \\ \text { Pa } & \text { Pascal } \\ \text { PVC } & \text { Polyvinylchlorid (Kunststoff) } \\ \text { RLF } & \text { Retrolinguale Fläche } \\ \text { ROSS } & \text { Repetitive Oral Suction Swallowing } \\ \text { s } & \text { Sekunde } \\ \text { SI } & \text { Système International d'Unités (internationals Einheitensystem) } \\ \text { SNA } & \text { Spina Nasalis Anterior } \\ \text { SSZ } & \text { Saugschluckzyklus } \\ \text { TOES } & \text { Technik der transoralen Endsokopie } \\ \text { UMG } & \text { Universitätsmedizin Göttingen } \\ \text { V } & \text { Verbindung } \\ \text { Z.B. } & \text { zum Beispiel } \\ \text { ZMK } & \text { Zahn-, Mund- und Kieferheilkunde } \\ \text { ZRM } & \text { Zungenrepositionsmanöver } \\ \end{array}$




\subsection{Abbildungsverzeichnis}

Abbildung 1: Schema der orofazialen Anatomie - 1 -

Abbildung 2: Biofunktionelle Einheiten im schematisierten Mediosagittalschnitt _ - 4 -

Abbildung 3: BFV und BFK im schematisierten Mediosagittalschnitt - 6 -

Abbildung 4: Membrantrichterplatte - 9 -

Abbildung 5: Phasen des physiologischen Schluckvorgangs - 12 -

Abbildung 6: Modifizierte Membrantrichterplatte - 26 -

Abbildung 7: Schemat. Darstellung des intra- und extraoralen Appl.- u. Messsystems - 28 -

Abbildung 8: Räumlicher Aufbau des Applikations- und Messsystems $\quad$ - 28 -

Abbildung 9: Handdruckmessgerät GMH $3156 \quad$ - 29 -

Abbildung 10: Magnetresonanztomograph Magnetom Tim Trio - 30 -

Abbildung 11: Druckdiagramm eines Probanden - 32 -

Abbildung 12: Auswahl von 4 repräsentativen Aktivitäts-Bildern eines SSZ - 34 -

Abbildung 13: MRT-Bild eines Probanden 37 -

Abbildung 14: Darstellung der Distanz zwischen SNA und IM $\quad 42$ -

Abbildung 15: Darstellung der mediosagittalen Flächengröße des Saugraums — - 43 -

Abbildung 16: Darstellung der mediosagittalen, retrolingualen Flächengröße $\quad$ - 44 -

Abbildung 17: Darstellung der mediosagittalen, epipharyngealen Flächengröße ～- 45 -

Abbildung 18: Darstellung der mediosagittalen, externen Mundbodenflächengröße $\quad$ - 46 -

\subsection{Tabellenverzeichnis}

Tabelle 1: Druckmittelwerte der Amplitudenmaxima - 40 -

Tabelle 2: Mittelwerte der erhobenen Längen- und Flächendaten - 41 - 


\subsection{Unterlagen zur ethischen Begutachtung}

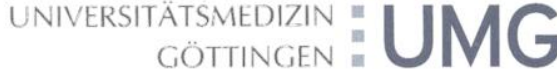

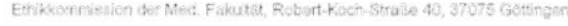

Herrn

PD Dr. med. dent. Michael Knösel

Abt. Kieferorthopädie

im Hause

vorab per Fax: 8350

Antragsnummer:

Studientitel

Antragsteller:
$12 / 11 / 09$ (bitte stets angeben)

Magnetresonanztomographische und simultane manometrische Untersuchungen zur intraoralen Kompartimentbildung beim

Schluckakt und in Ruhe

PD Dr. med. dent. Michael Knösel, Abt. Kieferorthopädie, Dr. med. Marios Psychogieos, Abt. Neuroradiologie. Prof. Dr. Dr.

med. W. Engelke, Abt. Kieferchirurgie, ZA Jajo Rasing, Abt.

Prothetik, UMG

Sehr geehrter Herr PD Dr. Knösel, sehr geehrte Damen und Herren,

nach Ergänzung der vorliegenden Dokumente und Beantwortung der im vorläufigen Votum aufgeführten Fragen durch Ihr Schreiben vom 3.2.2010 bestehen nunmehr keine ethischen und rechtlichen Bedenken gegen die Durchführung des oben genannten Forschungsvorhabens.

Wir wünschen viel Erfolg bei der Durchführung Ihres Projektes.

Wir empfehlen Ihnen den Datenschutzpassus der Einverständniserklärung optisch (Schriftart „Fett") eindeutiger hervorzuheben.

Die Ethik-Kommission weist darauf hin, dass die ärztliche und juristische Verantwortung bei den jeweiligen Prüfärzten verbleibt.

Auf die Einhaltung einschlägiger Gesetze und Rechtsvorschriften wird hingewiesen. Die nach Rechtslage notwendigen Unterrichtungen (u. A. Prüfplanänderungen, entsprechende Zwischenfallsereignisse, neue Datenlage, Nachmeldung von Prüfzentren, Abschlussbericht) sind der Ethik-Kommission unverzüglich vorzulegen.

Die Ethik-Kommission bestätigt, dass sie auf Grundlage nationaler Gesetze, Vorschriften sowie der GCP/ICH-Richtlinie arbeitet.

Mit freundlichen Grüßen

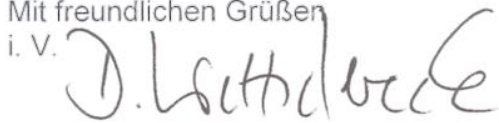

Prof. Dr. med. J. Brockmöller

Vorsitzender der Ethik-Kommission 


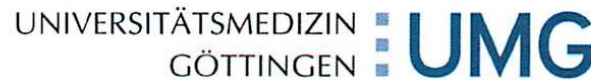

Ethikkommission der Med. Fakultät, Robert-Koch-Straße 40, 37075 Göttingen

Herrn

PD Dr. med. dent. Michael Knösel

Abt. Kieferorthopädie

im Hause

vorab per Fax: 8350

Nachrichtlich an: Johannes GlombekJglombek@aol.com
Medizinische Fakultät

Ethikkommission

Vorsitzender: Prof. Dr. Jürgen Brockmöller

Referentin

Regierungsrätin Doris Wettschereck

0551 / 39-8644 Telefon

37099 Göttingen Briefpost

Robert-Koch-Straße 40, 37075 Göttingen

Adresse

$0551 / 39-6629$ Telefon

0551 / 39-9536 Fax

ethik@med.uni-goettingen.de E-Mail

www.ethikkommission.med.uni-goettingen.de

27.09.2011 sc - gö Datum

Antragsnummer: $\quad 12 / 11 / 09$ (bitte stets angeben)

Studientitel: Magnetresonanztomographische und simultane manometrische

Untersuchungen zur intraoralen Kompartimentbildung beim Schluckakt

und in Ruhe

Antragsteller: $\quad$ PD Dr. med. dent. Michael Knösel, Abt. Kieferorthopädie, Dr. med.

Marios Psychogieos, Abt. Neuroradiologie, Prof. Dr. Dr. med. W.

Engelke, Abt. Kieferchirurgie, ZA Jajo Rasing, Petra Santander, Abt.

Prothetik, UMG

Doktorand : Johannes Glombek

Sehr geehrter Herr Dr. Knösel, sehr geehrte Damen und Herren,

wir bestätigen den Eingang des Antrags - Nachmeldung von Prüfärzten/Antragstellern - mit Schreiben vom 19.9.2011.

Von Seiten der Ethik-Kommission der Medizinischen Fakultät der Georg-August-Universität Göttingen bestehen keine Bedenken gegen die Einbeziehung der unten genannten Prüfärzte in die o.g. Studie:

Frau Petra Santander, Herr Johannes Glombek, Abt. Kieferchirurgie, Universitätsmedizin Göttingen Mit freundlichen Grüßen

$$
\therefore \text {. shell }
$$

Prof. Dr. med. J. Brockmöller

Vorsitzender der Ethik-Kommission 


\subsection{Aufklärungsunterlagen}

\section{Probanden - Informationsblatt}

\section{Magnetresonanztomographische und simultane manometrische Untersuchungen zur intraoralen Kompartimentbildung beim Schluckakt und in Ruhe}

\section{Projektleiter, Verantwortlichkeiten und Anschriften:}

Leiter der Klinischen Prüfung :

Priv. - Doz. Dr. med. dent. Michael Knösel

Abteilung für Kieferorthopädie, Zentrum für Zahn-, Mund- und Kieferheilkunde der Universitätsmedizin Göttingen, Georg-August Universität; e-mail: mknoesel@yahoo.de ; Telefon:39-22017

Sehr geehrte Probandin, sehr geehrter Proband,

das Verhalten der Weichteile im Mundbereich beim Schlucken ist ein unzureichend untersuchter Vorgang. Wir möchten Sie hiermit um Erlaubnis zu einer freiwilligen Untersuchung der Zungen-, Lippen-, Wangen-, Rachen-Muskulatur bitten, deren Ergebnisse uns wesentliche Erkenntnisse bei der Behandlung von Schluckstörungen vermitteln sollen. Beim normalen Schluckvorgang entstehen nach unserem Kenntnisstand im Munde abgetrennte Kammern mit leichtem Unterdruck. Uns interessiert die Fragestellung, ob dieser Druck im Bereich der Mundhöhle an verschiedenen Stellen variiert. Zum jetzigen Kenntnisstand ist das Vorliegen eines geringen Unterdruckes in der Mundhöhle als normal anzusehen. Uns interessiert daher die Fragestellung, wie die Weichteile im Mund-/ Gesichtsbereich auf eine leichte Störung dieses Unterdruckes reagieren, indem kurzzeitig durch eine kleine Schlauchverbindung ein Druckausgleich mit der Umgebung hergestellt wird.

Zunächst füllen Sie bitte den beiliegenden Fragebogen sorgfältig aus, da er uns wertvolle Hinweise gibt auf mögliche Risiken Ihrer Behandlung.

Die Untersuchung im Mund wird mit einem kleinen Messgerät durchgeführt, das Signale über einen in den Mund geführten Gummischlauch aufzeichnet. Sie erhalten dazu eine kleine Schiene, die auf die Zahnreihe eingesetzt wird. Die Untersuchung beinhaltet für Sie keinerlei Risiko und dauert 5 Minuten. Im Vorfeld der Untersuchung wird bei Ihnen eine Abformung des Oberkiefers zur Herstellung der Schiene durchgeführt. Während der Untersuchung ist die Schiene bei Ihnen im Mund eingesetzt und Sie führen normale Schluckbewegungen durch. Die Untersuchung wird in unserem Hause in einem Magnetresonanz-Tomographen (MRI) durchgeführt. Die Magnetresonanztomographie ist ein 
bildgebendes Verfahren, bei dem ein statisches Magnetfeld aufgebracht wird. Nach Abschalten des Magnetfeldes relaxieren die Atomkerne wieder in den Gleichgewichtszustand und geben dabei ein Radiofrequenzsignal ab. Dieses wird aufgezeichnet und durch zugeschaltete Magnetfeldgradienten räumlich zugeordnet. Aus dem aufgenommenen Signal lässt sich dann ein Bild ihres Mundbereichs rekonstruieren.

Vor dem Zutritt zum Untersuchungsraum wird die Testperson aufgefordert, alle magnetischen und metallischen Teile abzulegen, inklusive aller Teile, deren Magnetisierbarkeit unklar ist. Dies betrifft insbesondere Uhr, Brieftasche, Geldbörse, Schmuck, Zahnprothesen, Brille, Hörgerät, Haarspangen, Kugelschreiber, Scheckkarten und andere magnetisch kodierte Datenträger.

Mögliche Risiken:

- Verletzungen durch das Einbringen von metallischen, vor allem magnetisierbaren Gegenständen in den Untersuchungsraum. Die Testperson wird diesbezüglich aufgeklärt und direkt vor dem Betreten des Untersuchungsraumes von dem Untersuchenden nochmals aufgefordert, sich entsprechend dieser Maßgabe zu kontrollieren.

- Gewebeerwärmung durch überhöhte Radiofrequenzbelastung. Zur Überwachung dieses Gefahrenpotentials ist vom Hersteller des MR-Tomographen ein konservativer SAR-Monitor (SAR: Specific Absorption Rate) implementiert, der die Radiofrequenzbelastung überwacht und vom Benutzer nicht umgangen werden kann. Der SAR-Monitor unterbindet weitere Messungen, sobald die werkseingestellten gesetzeskonformen Grenzwerte überschritten werden.

- Die Exposition der Testperson in einem starken Magnetfeld hat nach heutigem Kenntnisstand keine schädigenden Effekte. Dennoch wird darauf geachtet, dass eine Testperson nur maximal einmal in einem Zeitraum von drei Tagen an einer MR-Untersuchung zu Forschungszwecken teilnimmt.

Die Teilnahme an der Aufklärung wird von der Testperson und dem Durchführenden der Aufklärung per Unterschrift dokumentiert. Die Testperson erhält eine Kopie des Frage- und des Informationsbogens, sowie der Einverständniserklärung.

Die Teilnahme an der Untersuchung ist freiwillig. Die Prüfärzte entscheiden über die Eignung für die Studie. Sie haben jederzeit das Recht, ohne Angabe von Gründen von der Teilnahme an der Studie zurückzutreten. Dabei entstehen Ihnen keinerlei Nachteile.

Die erhobenen Daten werden in anonymisierter Form gespeichert und dienen als Grundlage für eine wissenschaftliche Publikation.

Priv. - Doz. Dr. M. Knösel, Abteilung Kieferorthopädie 


\section{Prüfbogen}

\section{Patientenname :}

\begin{tabular}{|l|l|}
\hline \multicolumn{1}{|c|}{ Vorhaben } & \\
\hline 1. Anamnese & \\
\hline 2. oraler Befund & \\
\hline 3. Patientenaufklärung & \\
\hline 4. Patienteneinwilligung & \\
\hline 5. Abdrucknahme Oberkiefer & \\
\hline 6. Herstellung Positionierungsschiene & \\
\hline 7. Untersuchung im MRT & \\
\hline 8. Auswertung Manometrie/MRT & \\
\hline
\end{tabular}

Prüfarzt 
Robert - Koch - Straße 40

37099 Göttingen

Telefon: (0551) 39-22017

Zentrale: (0551) 39-0

e-mail:

mknoesel@yahoo.de

\section{Einverständniserklärung der Probanden}

\section{Magnetresonanztomographische und simultane manometrische Untersuchungen zur intraoralen Kompartimentbildung beim Schluckakt und in Ruhe}

Ich bin am von Herrn PD Dr. M. Knösel / Herrn Prof. Dr. Dr. W. Engelke / Herrn ZA H. Rasing / Dr. M. Psychogios (nicht Zutreffendes streichen) über Art und Durchführung der Untersuchung und Monitoring mit dem Druckmesssystem und dem MRT unterrichtet worden und habe das dazugehörige Informationsblatt gelesen. Über die darin beschriebene Anwendung des Druckmesssystems und die genannten Untersuchungsverfahren wurde ich in allem Umfang aufgeklärt. Ich habe zur Untersuchung und zur Durchführung keine weiteren Fragen und erkläre hiermit mein Einverständnis zu der besprochenen Untersuchung und erkläre mich mit der Behandlung einverstanden. Das hier gegebene Einverständnis kann ich jederzeit und ohne medizinische Folgen für die Behandlung widerrufen.

Ich erkläre mich einverstanden, dass die im Rahmen der bei mir durchgeführten Untersuchung ermittelten Daten in anonymisierter, d. $h$. nicht personenbezogener Form für wissenschaftliche Zwecke verwendet werden können.

Göttingen, den

(Unterschrift des Probanden) 


\section{MRT / Manometrie-Studie}

\section{Erfassungsbogen}

Datum:

Name:

Geburtsdatum:

Adresse:

Telefon:

Laufende $\mathrm{Nr}$ :

1. ANAMNESE

\begin{tabular}{|l|l|l|}
\hline & Ja & Nein \\
\hline Allergien? & & \\
\hline Bekannte Überempfindlichkeiten bei Anwendung von Kunststoffen? & & \\
\hline Herzerkrankungen? & & \\
\hline Organtransplantation? & & \\
\hline Immunsuppression? & & \\
\hline Nerven- oder Anfallsleiden? & & \\
\hline Infektionskrankheiten? & & \\
\hline Nierenerkrankungen? & & \\
\hline Beschwerden an den Zähnen oder dem Zahnfleisch? & & \\
\hline Sonstige Erkrankungen? & & \\
\hline Schwangerschaft? & \\
\hline
\end{tabular}

2. INTRAORALER BEFUND (durch den Prüfarzt)

\begin{tabular}{|l|l|l|}
\hline & Ja & Nein \\
\hline Klasse I Situation ? & & \\
\hline Offener Biss? & & \\
\hline Physiologischer Schluckvorgang ? & & \\
\hline Lückensituation? & & \\
\hline
\end{tabular}

Datum, Unterschrift Proband

Datum, Unterschrift Prüfarzt 


\subsection{Datentabellen der Ergebnisse}

MRT: Einzelwerte aller Probanden

\begin{tabular}{|c|c|c|c|c|c|c|c|}
\hline Proband & $\begin{array}{l}\text { Saugschluck- } \\
\text { zyklus }\end{array}$ & Aktivität & Distanz (cm) & $\begin{array}{l}\text { Saug- } \\
\text { raum }\left(\mathrm{cm}^{2}\right)\end{array}$ & $\operatorname{RLF}\left(\mathrm{cm}^{2}\right)$ & $\operatorname{EPF}\left(\mathrm{cm}^{2}\right)$ & EMF $\left(\mathrm{cm}^{2}\right)$ \\
\hline 1 & 1 & 1 & 5,83 & 2,519 & 4,425 & 8,735 & 13,321 \\
\hline 1 & 2 & 1 & 5,957 & 2,418 & 6,504 & 9,207 & 13,024 \\
\hline 1 & 3 & 1 & 5,526 & 1,646 & 6,084 & 8,89 & 13,355 \\
\hline 1 & 1 & 2 & 5,696 & 1,324 & 5,402 & 9,181 & 11,286 \\
\hline 1 & 2 & 2 & 5,712 & 2,735 & 5,406 & 9,111 & 12,368 \\
\hline 1 & 3 & 2 & 5,838 & 4,169 & 4,391 & 10,161 & 10,717 \\
\hline 1 & 1 & 3 & 5,712 & 3,885 & 5,135 & 11,211 & 10,705 \\
\hline 1 & 2 & 3 & 5,593 & 3,567 & 5,373 & 10,978 & 11,082 \\
\hline 1 & 3 & 3 & 5,691 & 6,312 & 5,497 & 14,166 & 13,274 \\
\hline 1 & 1 & 4 & 5,537 & & & 2,094 & 14,175 \\
\hline 1 & 2 & 4 & 5,56 & & & 3,632 & 12,702 \\
\hline 1 & 3 & 4 & 5,738 & & & 2,636 & 12,488 \\
\hline 2 & 1 & 1 & 7,28 & 5,489 & 5,693 & 9,571 & 18,309 \\
\hline 2 & 2 & 1 & 7,381 & 5,634 & 5,875 & 8,743 & 19,356 \\
\hline 2 & 3 & 1 & 7,182 & 2,965 & 6,405 & 9,707 & 21,301 \\
\hline 2 & 1 & 2 & 7,056 & 4,032 & 7,527 & 10,301 & 21,398 \\
\hline 2 & 2 & 2 & 7,16 & 5,471 & 8,588 & 11,172 & 19,998 \\
\hline 2 & 3 & 2 & 7,029 & 4,85 & 8,364 & 11,059 & 20,602 \\
\hline 2 & 1 & 3 & 7,018 & 4,657 & 6,953 & 10,886 & 20,946 \\
\hline 2 & 2 & 3 & 7,164 & 5,583 & 9,085 & 11,497 & 19,332 \\
\hline 2 & 3 & 3 & 7,075 & 5,797 & 8,787 & 11,685 & 19,996 \\
\hline 2 & 1 & 4 & 7,079 & & & 3,941 & 21,349 \\
\hline 2 & 2 & 4 & 7,11 & & & 2,623 & 24,054 \\
\hline 2 & 3 & 4 & 7,049 & & & 3,204 & 23,136 \\
\hline 3 & 1 & 1 & 6,468 & 2,225 & 6,014 & 8,221 & 21,395 \\
\hline 3 & 2 & 1 & 6,364 & 4,69 & 6,472 & 7,778 & 21,085 \\
\hline 3 & 3 & 1 & 6,621 & 3,576 & 5,746 & 7,908 & 21,973 \\
\hline 3 & 1 & 2 & 6,308 & 3,033 & 5,257 & 8,371 & 21,933 \\
\hline 3 & 2 & 2 & 6,472 & 3,415 & 5,255 & 8,089 & 22,26 \\
\hline 3 & 3 & 2 & 6,303 & 2,923 & 5,473 & 8,001 & 22,178 \\
\hline 3 & 1 & 3 & 6,272 & 3,744 & 5,319 & 8,586 & 21,692 \\
\hline 3 & 2 & 3 & 6,42 & 3,945 & 4,629 & 8,309 & 22,518 \\
\hline 3 & 3 & 3 & 6,306 & 3,223 & 5,404 & 8,295 & 22,368 \\
\hline 3 & 1 & 4 & 6,427 & & & 3,492 & 21,359 \\
\hline 3 & 2 & 4 & 6,33 & & & 3,285 & 21,591 \\
\hline 3 & 3 & 4 & 6,06 & & & 3,053 & 21,46 \\
\hline
\end{tabular}




\begin{tabular}{|c|c|c|c|c|c|c|c|}
\hline 4 & 1 & 1 & 7,4 & 4,873 & 8,605 & 5,486 & 17,341 \\
\hline 4 & 2 & 1 & 7,602 & 2,891 & 9,057 & 5,901 & 18,338 \\
\hline 4 & 3 & 1 & 7,85 & 7,784 & 7,266 & 5,776 & 15,258 \\
\hline 4 & 1 & 2 & 7,274 & 3,348 & 8,1 & 5,971 & 17,862 \\
\hline 4 & 2 & 2 & 7,397 & 4,916 & 8,194 & 7,144 & 18,575 \\
\hline 4 & 3 & 2 & 7,31 & 3,39 & 9,142 & 6,793 & 18,834 \\
\hline 4 & 1 & 3 & 7,32 & 4,577 & 6,751 & 6,887 & 17,792 \\
\hline 4 & 2 & 3 & 7,349 & 5,966 & 7,342 & 6,983 & 18,276 \\
\hline 4 & 3 & 3 & 7,256 & 5,075 & 9,498 & 7,304 & 17,632 \\
\hline 4 & 1 & 4 & 7,084 & & & 2,219 & 19,511 \\
\hline 4 & 2 & 4 & 7,174 & & & 2,891 & 18,671 \\
\hline 4 & 3 & 4 & 7,062 & & & 2,283 & 21,612 \\
\hline 5 & 1 & 1 & 6,889 & 5,677 & 7,222 & 9,316 & 16,738 \\
\hline 5 & 2 & 1 & 6,771 & 4,336 & 7,363 & 9,571 & 16,129 \\
\hline 5 & 3 & 1 & 7,308 & 3,969 & 7,865 & 9,687 & 15,645 \\
\hline 5 & 1 & 2 & 6,651 & 4,805 & 6,577 & 10,207 & 16,301 \\
\hline 5 & 2 & 2 & 6,732 & 2,911 & 7,962 & 10,003 & 16,965 \\
\hline 5 & 3 & 2 & 6,979 & 5,263 & 7,15 & 10,23 & 15,912 \\
\hline 5 & 1 & 3 & 6,653 & 5,597 & 6,806 & 10,358 & 16,677 \\
\hline 5 & 2 & 3 & 6,717 & 3,754 & 7,524 & 10,268 & 16,911 \\
\hline 5 & 3 & 3 & 7,156 & 5,988 & 6,846 & 11,109 & 16,345 \\
\hline 5 & 1 & 4 & 6,679 & & & 2,987 & 17,454 \\
\hline 5 & 2 & 4 & 6,601 & & & 4,352 & 16,373 \\
\hline 5 & 3 & 4 & 7,002 & & & 2,647 & 16,435 \\
\hline 6 & 1 & 1 & 6,269 & 1,964 & 3,951 & 7,742 & 13,653 \\
\hline 6 & 2 & 1 & 6,381 & 4,424 & 4,163 & 8,038 & 14,989 \\
\hline 6 & 3 & 1 & 6,674 & 3,352 & 5,214 & 8,985 & 13,591 \\
\hline 6 & 1 & 2 & 6,459 & 8,105 & 4,228 & 9,343 & 11,056 \\
\hline 6 & 2 & 2 & 6,508 & 7,188 & 4,807 & 9,048 & 12,983 \\
\hline 6 & 3 & 2 & 6,669 & 7,115 & 5,975 & 9,607 & 13,154 \\
\hline 6 & 1 & 3 & 6,552 & 8,687 & 3,93 & 9,246 & 11,359 \\
\hline 6 & 2 & 3 & 6,522 & 7,856 & 4,616 & 8,824 & 13,363 \\
\hline 6 & 3 & 3 & 6,742 & 7,837 & 5,933 & 10,065 & 12,973 \\
\hline 6 & 1 & 4 & 6,399 & & & 1,207 & 14,334 \\
\hline 6 & 2 & 4 & 6,423 & & & 1,507 & 14,305 \\
\hline 6 & 3 & 4 & 6,601 & & & 2,021 & 15,192 \\
\hline 7 & 1 & 1 & 7,533 & 3,064 & 5,601 & 9,493 & 9,767 \\
\hline 7 & 2 & 1 & 7,521 & 0,647 & 5,36 & 8,75 & 10,595 \\
\hline 7 & 3 & 1 & 7,213 & 5,977 & 5,1 & 9,626 & 9,941 \\
\hline 7 & 1 & 2 & 7,32 & 8,804 & 3,546 & 9,322 & 10,33 \\
\hline 7 & 2 & 2 & 7,358 & 6,646 & 3,615 & 9,592 & 10,326 \\
\hline 7 & 3 & 2 & 7,412 & 7,516 & 3,436 & 10,013 & 9,891 \\
\hline 7 & 1 & 3 & 7,388 & 9,718 & 4,035 & 9,938 & 10,014 \\
\hline 7 & 2 & 3 & 7,486 & 7,332 & 3,344 & 10,148 & 10,181 \\
\hline
\end{tabular}




\begin{tabular}{|c|ccccccc|}
\hline 7 & 3 & 3 & 7,363 & 8,655 & 3,284 & 10,246 & 9,846 \\
\hline 7 & 1 & 4 & 7,415 & & & 3,132 & 10,799 \\
\hline 7 & 2 & 4 & 7,454 & & & 3,37 & 10,476 \\
\hline 7 & 3 & 4 & 7,375 & & & 3,781 & 10,006 \\
\hline 8 & 1 & 1 & 7,667 & 4,729 & 9,904 & 11,871 & 15,944 \\
\hline 8 & 2 & 1 & 7,921 & 1,845 & 8,786 & 11,743 & 15,314 \\
\hline 8 & 3 & 1 & 7,415 & 5,996 & 9,651 & 12,286 & 15,68 \\
\hline 8 & 1 & 2 & 7,602 & 7,103 & 8,854 & 13,696 & 15,137 \\
\hline 8 & 2 & 2 & 7,368 & 7,474 & 8,165 & 13,955 & 15,097 \\
\hline 8 & 3 & 2 & 7,365 & 6,483 & 8,828 & 13,497 & 15,396 \\
\hline 8 & 1 & 3 & 7,492 & 7,627 & 8,302 & 13,598 & 15,122 \\
\hline 8 & 2 & 3 & 7,435 & 8,585 & 7,329 & 14,162 & 15,278 \\
\hline 8 & 3 & 3 & 7,31 & 7,468 & 7,865 & 14,029 & 15,635 \\
\hline 8 & 1 & 4 & 7,564 & & & 2,797 & 15,358 \\
\hline 8 & 2 & 4 & 7,339 & & & 2,411 & 15,269 \\
\hline 8 & 3 & 4 & 7,246 & & & 3,89 & 16,085 \\
\hline 9 & 1 & 1 & 7,243 & 1,943 & 9,26 & 9,238 & 10,366 \\
\hline 9 & 2 & 1 & 7,155 & 2,639 & 10,768 & 10,357 & 9,709 \\
\hline 9 & 3 & 1 & 7,492 & 9,809 & 10,252 & 10,507 & 8,637 \\
\hline 9 & 1 & 2 & 7,17 & 4,757 & 7,12 & 11,225 & 10,253 \\
\hline 9 & 2 & 2 & 7,126 & 5,576 & 8,295 & 11,857 & 9,545 \\
\hline 9 & 3 & 2 & 7,138 & 2,519 & 10,568 & 12,651 & 8,549 \\
\hline 9 & 1 & 3 & 7,501 & 5,937 & 6,64 & 12,067 & 8,798 \\
\hline 9 & 2 & 3 & 7,171 & 5,959 & 8,435 & 11,93 & 9,054 \\
\hline 9 & 3 & 3 & 7,065 & 3,638 & 9,515 & 13,284 & 8,494 \\
\hline 9 & 1 & 4 & 7,197 & & & 1,821 & 12,568 \\
\hline 9 & 4 & 7,112 & & & 4,491 & 12,753 \\
\hline & 3 & 7,136 & & & 5,644 & 12,222 \\
\hline & 2 & 4 & & & & & \\
\hline
\end{tabular}

Manometrie: Einzelwerte aller Probanden

\begin{tabular}{|cccc|}
\hline Proband & $\begin{array}{c}\text { Saugschluck } \\
\text { zyklus }\end{array}$ & Position & Amplituden - Maximum (mbar) \\
\hline 1 & 1 & Gaumen & $-115,4$ \\
\hline 1 & 1 & Wange & $-40,4$ \\
\hline 1 & 2 & Gaumen & $-94,9$ \\
\hline 1 & 2 & Wange & $-47,9$ \\
\hline 1 & 3 & Gaumen & $-70,2$ \\
\hline 1 & 3 & Wange & $-38,1$ \\
\hline 2 & 1 & Gaumen & $-106,8$ \\
\hline 2 & 1 & Wange & $-111,8$ \\
\hline 2 & 2 & Gaumen & $-130,3$ \\
\hline 2 & 2 & Wange & -131 \\
\hline
\end{tabular}




\begin{tabular}{|c|c|c|c|}
\hline 2 & 3 & Gaumen & $-113,1$ \\
\hline 2 & 3 & Wange & $-132,5$ \\
\hline 3 & 1 & Gaumen & $-103,2$ \\
\hline 3 & 1 & Wange & $-34,4$ \\
\hline 3 & 2 & Gaumen & $-110,8$ \\
\hline 3 & 2 & Wange & $-54,1$ \\
\hline 3 & 3 & Gaumen & $-100,1$ \\
\hline 3 & 3 & Wange & $-11,6$ \\
\hline 4 & 1 & Gaumen & $-40,6$ \\
\hline 4 & 1 & Wange & $-29,6$ \\
\hline 4 & 2 & Gaumen & $-49,2$ \\
\hline 4 & 2 & Wange & $-67,5$ \\
\hline 4 & 3 & Gaumen & $-31,7$ \\
\hline 4 & 3 & Wange & $-23,6$ \\
\hline 5 & 1 & Gaumen & $-65,4$ \\
\hline 5 & 1 & Wange & $-60,5$ \\
\hline 5 & 2 & Gaumen & $-113,3$ \\
\hline 5 & 2 & Wange & $-57,7$ \\
\hline 5 & 3 & Gaumen & -108 \\
\hline 5 & 3 & Wange & $-30,2$ \\
\hline 6 & 1 & Gaumen & $-87,5$ \\
\hline 6 & 1 & Wange & $-89,3$ \\
\hline 6 & 2 & Gaumen & $-69,5$ \\
\hline 6 & 2 & Wange & $-85,2$ \\
\hline 6 & 3 & Gaumen & $-86,1$ \\
\hline 6 & 3 & Wange & $-88,4$ \\
\hline 7 & 1 & Gaumen & $-94,3$ \\
\hline 7 & 1 & Wange & $-60,5$ \\
\hline 7 & 2 & Gaumen & $-111,1$ \\
\hline 7 & 2 & Wange & $-77,3$ \\
\hline 7 & 3 & Gaumen & $-84,6$ \\
\hline 7 & 3 & Wange & $-92,3$ \\
\hline 8 & 1 & Gaumen & $-81,5$ \\
\hline 8 & 1 & Wange & -78 \\
\hline 8 & 2 & Gaumen & $-81,3$ \\
\hline 8 & 2 & Wange & $-77,1$ \\
\hline 8 & 3 & Gaumen & $-63,1$ \\
\hline 8 & 3 & Wange & $-75,1$ \\
\hline 9 & 1 & Gaumen & -119 \\
\hline 9 & 1 & Wange & $-95,1$ \\
\hline 9 & 2 & Gaumen & $-87,2$ \\
\hline 9 & 2 & Wange & $-68,5$ \\
\hline 9 & 3 & Gaumen & $-82,5$ \\
\hline 9 & 3 & Wange & $-71,5$ \\
\hline
\end{tabular}




\section{Literaturverzeichnis}

\section{Abrams IN (1963):}

Oral muscle pressures.

Angle Orthod 33, 83-104

\section{Akin E, Sayin MÖ, Karacay S, Bulakbasi N (2006):}

Real-time balanced turbo field echo cine-magnetic resonance imaging evaluation of tongue movements during deglutition in subjects with anterior open bite.

Am J Orthod Dentofacial Orthop 129, 24-28

\section{Anagnostara A, Stoeckli S, Weber OM, Kollias SS (2001):}

Evaluation of the anatomical and functional properties of deglutition with various kinetic high-speed MRI sequences.

J Magn Reson Imaging 14, 194-199

\section{Angle EH (1907):}

Treatment of malocclusion of the teeth.

7th edn. White, Philadelphia

\section{Ballard CF (1966):}

The adaptive alveolar processes.

Ann R Coll Surg Engl 39, 299-311

\section{Bartolome G, Schröter-Morasch H:}

Schluckstörungen: Diagnostik und Rehabilitation.

4. Auflage; Urban \& Fischer Verlag, München 2010

\section{Böhme G (Hrsg.):}

Sprach-, Sprech-, Stimm- und Schluckstörungen, Band 1.

Urban \& Fischer Verlag, München 2003 


\section{Böhme G, Benecke P, Engelmann I, Feneberg W, Penner H:}

Sprach-, Sprech-, Stimm- und Schluckstörungen, Band 2.

Urban \& Fischer Verlag, München 2006

\section{Breustedt A (1970):}

Problems of the resting position of the tongue.

Dtsch Zahn Mund Kieferheilkd Zentralbl 54, 308-319

Breyer T, Echternach M, Arndt S, Richter B, Speck O, Schumacher M, Markl M (2009):

Dynamic magnetic resonance imaging of swallowing and laryngeal motion using parallel imaging at $3 \mathrm{~T}$.

Magn Reson Imaging 27, 48-54

\section{Butler SG, Stuart A, Kemp S (2009):}

Flexible endoscopic evaluation of swallowing in healthy young and older adults.

Ann Otol Rhinol Laryngol 118, 99-106

\section{Chi-Fishman G (2005):}

Quantitative lingual, pharyngeal and laryngeal ultrasonography in swallowing research: a technical review.

Clin Linguist Phon $\underline{19}, 589-604$

\section{Dauber W, Feneis, H:}

Bild-Lexikon der Anatomie.

9. Auflage; Georg Thieme Verlag, Stuttgart 2005

\section{Delaney AL, Arvedson JC (2008):}

Development of swallowing and feeding: prenatal through first year of life.

Dev Disabil Res Rev 14, 105-117

\section{Dodds WJ, Stewart ET, Logemann JA (1990):}

Physiology and radiology of the normal oral and pharyngeal phases of swallowing. Am J Roentgenol 154, 953-963 


\section{Eckert-Möbius A (1953):}

The importance of the tongue for nasal and oral respiration.

Fortschr Kieferorthop 14, 229-239

\section{Engelke W:}

Systematische Rhonchopathiebehandlung in der zahnärztlichen Praxis.

Cuvillier-Verlag, Göttingen 2007

\section{Engelke W (2003):}

Ein Manöver zur Positionierung der Zunge am Gaumen.

Sprache Stimme Gehör 27, 171-175

\section{Engelke W (2009):}

Biofunktionelle Therapie (BFT) - ein neuer Zugang zur Behandlung orofazialer Dysfunktionen.

Zahn Prax 12, 314-323

\section{Engelke W, Hoch G (1999):}

Funktionsdiagnostik mit intraoralen Sensoren.

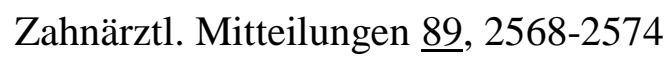

\section{Engelke W, Hoch G, Schwestka-Polly R (1995a):}

Electromagnetic articulography as a method for detecting the influence of spikes on tongue movement.

Eur J Orthod 17, 411-417

\section{Engelke W, Müller C, Petersen Ch (1995b):}

Elektromagnetische Untersuchungen zur Physiologie oropharyngealer Schluckbewegungen.

Sprache Stimme Gehör 19, 105-113

\section{Engelke W, Engelhardt W, Mendoza-Gärtner M, Deccó O, Barriero J, Knösel M (2010):}

Functional treatment of snoring based on the tongue-repositioning manouvre.

Eur J Orthod 32, 490-495 


\section{Engelke W, Jung K, Knösel M (2011):}

Intra-oral compartment pressures: a biofunctional model and experimental measurements under different conditions of posture.

Clin Oral Investig $\underline{15}, 165-176$

\section{Faigenblum MJ (1966):}

Negative oral pressures. A research report.

Dent Pract Dent Rec 16, 214-216

\section{Feinberg M, Knebl J, Tully J, Segall L (1990):}

Aspiration and the elderly.

Dysphagia $\underline{5}, 61-71$

\section{Foucart JM, Carpentier P, Pajoni D, Rabischong P, Pharaboz C (1998):}

Kinetic magnetic resonance imaging analysis of swallowing: a new approach to pharyngeal function.

Surg Radiol Anat 20, 53-55

\section{Fränkel G:}

Manometrische und endoskopische Diagnostik oropharyngealer Dysphagien.

Med. Diss. Göttingen 2012

\section{Fränkel R (1967):}

The dynamics of the interocclusal negative pressure.

Deutsche Zahnärztl. Zeitschrift 22, 282-290

\section{Friedrich G, Bigenzahn W:}

Phoniatrie.

Hans Huber Verlag, Bern 1995

Fröhlich K, Thüer U, Ingerwall B (1991):

Pressure from the tongue on the teeth in young adults.

Angle Orthod $\underline{61}, 17-24$ 
German R, Crompton A, Levitch L, Thexton A (1992):

The mechanism of suckling in two species of infant mammal: miniature pigs and long-tailed macaques.

J Exp Zool 261, 322-330

Gingrich LL, Stierwalt JA, Hageman CF, LaPointe LL (2012):

Lingual propulsive pressures across consistencies generated by the anteromedian and posteromedian tongue by healthy young adults.

J Speech Lang Hear Res $\underline{55}$, 960-972

\section{Grau T:}

Ultraschall in der Anästhesie und Intensivmedizin: Lehrbuch der Ultraschalldiagnostik.

Deutscher Ärzte-Verlag, Köln 2009

\section{Hartl DM, Albiter M, Kolb F, Luboinski B, Sigal R (2003):}

Morphologic parameters of normal swallowing events using single-shot fast spin echo dynamic MRI.

Dysphagia $\underline{18}, 255-262$

\section{Hiss SG, Postma GN (2003):}

Fiberoptic endoscopic evaluation of swallowing.

Laryngoscope $\underline{113}, 1386-1393$

\section{Howell A, Manly R (1948):}

An Electronic Strain gauge for Measuring Oral Forces.

J Dent Res 27, 705-712

\section{Howell A, Brudevold F (1950):}

Vertical forces used during chewing of food.

J Dent Res 29, 133-136 
Iida S, Harada T, Okamoto M, Inada Y, Kogo M, Masuda Y (2003):

Soft palate movement during sucking behavior.

Dysphagia $\underline{18}, 96-100$

\section{Kauffmann GW, Moser E, Sauer R:}

Radiologie.

3. Auflage; Urban \& Fischer Verlag, München 2006

Kennedy D, Kieser J, Bolter C, Swain M, Singh B, Waddell JN (2010):

Tongue pressure patterns during water swallowing.

Dysphagia 25, 11-19

Kieser J, Singh B, Swain M, Ichim I, Waddell JN, Kennedy D, Foster K, Livingstone V (2008):

Measuring intraoral pressure: adaptation of a dental appliance allows measurement during function.

Dysphagia 23, 237-243

\section{Körbitz A:}

Kursus der systematischen Orthodontik.

Johann Ambrosius Barth Verlag, Leipzig 1914

\section{Krysiński Z, Piotrowski P (1990):}

Negative pressure under upper complete dentures during the first half of their fitting.

Zahn Mund Kieferheilkd Zentralbl 모, 141-143

\section{Langmore S, Schatz K, Olson N (1988):}

Fiberoptic endoscopic evaluation of swallowing safety a new procedure.

Dysphagia $\underline{2}, 216-219$ 
Lazarus CL, Logemann JA, Pauloski BR, Rademaker AW, Larson CR, Mittal BB, Pierce M (2000):

Swallowing and tongue function following treatment for oral and oropharyngeal cancer.

J Speech Lang Hear Res 43, 1011-1023

\section{Lear CS, Morrees CF (1969):}

Buccolingual muscle force and dental arch form.

Am J Orthod 56, 379-393

\section{Lippert H:}

Lehrbuch Anatomie.

7. Auflage; Urban \& Fischer Verlag, München 2006

\section{Logemann JA (1988):}

Swallowing Physiology and Pathophysiology.

Otolaryngol Clin North Am 21, 613-623

\section{Logemann JA:}

Evaluation and treatment of swallowing disorders.

2. Auflage; PRO-ED Verlag, Austin-Texas 1998

\section{Logemann JA, Rademaker AW, Pauloski BR, Ohmae Y, Kahrilas PJ (1998):}

Normal Swallowing Physiology as Viewed by Videofluoroscopy and Videoendoscopy.

Folia Phoniatr Logop 50, 311-319

\section{Magendie F:}

Mémoire sur l'usage de l'épiglotte dans la déglutition.

Meguignon-Marvis Verlag, Paris 1813

Marik P, Kaplan D (2003):

Aspiration pneumonia and dysphagia in the elderly.

Chest $\underline{124}, 328-336$ 


\section{Matsuo K, Palmer JB (2009):}

Coordination of mastication, swallowing and breathing.

Jpn Dent Sci Rev $\underline{45}, 31-40$

\section{McConnel FM, Cerenko D, Mendelsohn MS (1988):}

Manofluorographic analysis of swallowing.

Otolaryngol Clin North Am 21, 625-635

\section{Melsen B, Attina L, Santuari M, Attina A (1987):}

Relationships between swallowing pattern, mode of respiration, and development of malocclusion.

Angle Orthod 28, 618-623

Mew JR (2004):

The postural basis of malocclusion: a philosophical overview.

Am J Orthod Dentofacial Orthop 126, 729-738

\section{Miller AJ (1982):}

Deglutition.

Physiol Rev $\underline{62}, 129-184$

\section{Nacci A, Ursino F, La Vela R, Matteucci F, Mallardi V, Fattori B (2008):}

Fiberoptic endoscopic evaluation of swallowing (FEES): proposal for informed consent. Acta Otorhinolaryngol Ital $\underline{28}, 206-211$

\section{Neuschäfer-Rube C, Wein BB, Angerstein W, Klajman S Jr, Fischer-Wein G (1997):}

Sector-related grey scale analysis of video ultrasound recorded tongue movements in swallowing.

HNO $\underline{45}, 556-562$

\section{Niitsu M, Kumada M, Campeau NG, Niimi S, Riederer SJ, Itai Y (1994):}

Tongue displacement: visualisation with rapid tagged magnetization-prepared MR imaging. Radiology 191, 578-580 
Nilsson H, Ekberg O, Olsson R, Kjellin O, Hindfelt B (1996):

Quantitative assessment of swallowing in healthy adults.

Dysphagia $\underline{11}, 110-116$

\section{Noltemeier H:}

Einführung in die allgemeine Kiefer- und Gesichtsorthopädie.

Alfeld, Hannover 1949

Ohkubo M, Sano T, Ishida R, Higaki T, Nishikawa K, Hayakawa Y, Otonari T, Yamamoto-Otonari M, Harada T, Wakoh M (2008):

Static MR images for diagnosis of swallowing.

Bull Tokyo Dent Coll 49, 113-119

Orchardson R, Cadden S (2009):

Mastication and swallowing: functions, performance and mechanisms.

Dent Update 36, 327-330, 332-334, 337

Panebianco V, Ruoppolo G, Pelle G, Schettino I, Roma R, Bernardo S, De Vincentiis C, Longo L, Passariello R (2010):

Morpho-functional patterns of physiologic oropharyngeal swallowing evaluated with dynamic fast MRI.

Eur Arch Otorhinolaryngol 267, 1461-1466

\section{Pasler FA:}

Zahnärztliche Radiologie, 2. Auflage.

Georg Thieme Verlag, Stuttgart 1989

\section{Peng CL, Jost-Brinkmann PG, Yoshida N, Chou HH, Lin CT (2004):}

Comparison of tongue functions between mature and tongue-thrust swallowing-an ultrasound investigation.

Am J Orthod Dentofacial Orthop $\underline{125}, 562-570$ 


\section{Pokieser P (2010):}

Videokinematographie des Schluckaktes: Ein Evergreen der funktionellen Radiologie.

ERG News 03/2010

\section{Pokieser P, Scharitzer M:}

The Clinical and Radiological Approach to Dysphagia; Radiology of the Pharynx and the Esophagus.

Springer Verlag, Berlin 2004

\section{Pokieser P, Schober E, Schima W (1995):}

Videocinematography of swallowing. Indications, methodology and findings.

Radiologe 35, 703-711

\section{Proffit W (1978):}

Equilibrium theory revisited: factors influencing position of the teeth.

Angle Orthod $\underline{48}, 175-186$

\section{Reiser M, Semmler W:}

Magnetresonanztomographie.

Springer-Verlag Berlin, Heidelberg 2002

\section{Reitemeier B, Schwenzer N, Ehrenfeld M:}

Einführung in die Zahnmedizin.

Georg-Thieme Verlag KG, Stuttgart 2006

\section{Robin DA, Goel A, Somodi LB, Luschei ES (1992):}

Tongue strength and endurance: relation to highly skilled movements.

J Speech Hear Res 35, 1239-1245

\section{Robbins J, Levine R, Wood J, Roecker EB, Luschei ES (1995):}

Age effects on lingual pressure generation as a risk factor for dysphagia.

J Gerontol A Biol Sci Med Sci 50, 257-262 


\section{Rugiu MG (2007):}

Role of videofluoroscopy in evaluation of neurologic dysphagia.

Acta Otorhinolaryngol 27, 306-316

\section{Santander P, Engelke W, Olthoff A, Völter C (2013):}

Intraoral pressure patterns during swallowing.

Eur Arch Otorhinolaryngol 270, 1019-1025

\section{Schröter-Morasch H, Bartolome G, Troppmann N, Ziegler W (1999):}

Values and limitations of pharyngolaryngoscopy (transnasal, transoral) in patients with dysphagia.

Folia Phoniatr Logop $\underline{51}$, 172-182

\section{Selley W, Ellis R, Flack F, Brooks W (1990):}

Coordination of sucking, swallowing and breathing in the newborn: its relationship to infant feeding and normal development.

Br J Disord Commun 25, 311-327

\section{Suto Y, Kamba M, Kato T (1995):}

Technical note: Dynamic analysis of the pharynx during swallowing using Turbo-FLASH magnetic resonance imaging combined with an oral positive contrast agent - a preliminary study.

Br J Radiol 요, 1099-1102

\section{Stein J, Wehrmann T:}

Funktionsdiagnostik in der Gastroenterologie.

Springer Medizin Verlag, Heidelberg 2006

\section{Tamine K, Ono T, Hori K, Kondoh J, Hamanaka S, Maeda Y (2010):}

Age-related changes in tongue pressure during swallowing.

J Dent Res 모, 1097-1101 


\section{Thexton A, Crompton A, Owerkowicz T, German R (2004):}

Correlation between intraoral pressures and tongue movements in the suckling pig.

Arch Oral Biol $\underline{49}, 567-575$

\section{Tränkmann J (1982):}

Orofaziale Dyskinesien.

Zahnärztl Prax $\underline{33}$, 474-478

\section{Vitorino J (2010):}

Effect of age on tongue strength and endurance scores of healthy Portugese speakers.

Int J Speech Lang Pathol 12, 237-243

\section{Vogl TJ:}

Kernspintomographie in der Kopf-Hals-Region.

Springer Verlag, Berlin 1991

\section{Wein B, Alzen G, Tolxdorff T, Böckler R, Klajman S, Huber W (1988):}

Computerized sonographic imaging of tongue motility using pseudo-3 dimensional reconstruction.

Ultraschall Med $\underline{9}, 95-97$

\section{Wein B, Angerstein W, Klajman S, Zimmermann R, Skopnik H (1993):}

Sonography of the Tongue and Pressure Measurements during Bottle Feeding of Newborn Infants. First Results.

Klin Padiatr 205, 103-106

\section{White R, Cotton SM, Hind J, Robbins J, Perry A (2009):}

A comparison of the reliability and stability of oro-lingual swallowing pressures in patients with head and neck cancer and healthy adults.

Dysphagia 24, 137-144 


\section{Winders R (1956):}

A study in the development of an electronic technique to measure the forces exerted on the dentition by the perioral and lingual musculature.

Am J Orthod $\underline{42}, 645-657$

\section{Zhang S, Olthoff A, Frahm J (2012):}

Real-Time Magnetic Resonance Imaging of Normal Swallowing.

Magn Reson Imaging $\underline{35}$, 1372-1379

\section{Zirkler HU:}

Polysensographische Untersuchung der orofazialen Basisfunktion im Wachzustand und während der Nachtruhe.

Med. Diss. Göttingen 2002 


\section{Danksagung}

Für die Vergabe des Themas, vor allem aber für die intensive Betreuung, bedanke ich mich in besonderer Weise bei Herrn Prof. Dr. Dr. Wilfried Engelke. Seine zahlreichen Anregungen und seine ausdauernde Unterstützung motivierten mich bei meiner wissenschaftlichen Arbeit sehr.

Frau Dr. Petra Santander danke ich für ihre kontinuierliche Hilfe, insbesondere in der Einarbeitungsphase in das behandelte Thema. Vor allen Dingen während der praktischen Versuchsdurchführungen stand sie mir freundlicherweise oft mit ihrem Rat zur Seite.

$\mathrm{Zu}$ weiterem Dank bin ich Herrn Dr. Marios Psychogios verpflichtet, da er bei der experimentellen Arbeitsphase die Bedienung des Magnetresonanztomographen übernahm.

Bei Herrn Simon Schneider und Herrn David Ellenberger aus der Abteilung für Medizinische Statistik möchte ich mich für ihre Hilfe bei der Auswertung der erhobenen Daten und der statistischen Analyse bedanken. 